\title{
Multiuser MIMO-OFDM for Next-Generation Wireless Systems
}

\author{
Multiple-Input Multiple-Output (MIMO) wireless systems using OFDM promise to \\ provide the needed performance for future consumer products. This paper reviews \\ existing MIMO-OFDM systems, discusses their limitations, and examines the \\ use of Genetic Algorithms (GAs) as a tool to handle large numbers of users.
}

By Ming Jiang, Member IEEE, And Lajos Hanzo, Fellow IEEE

ABSTRACT | This overview portrays the 40-year evolution of orthogonal frequency division multiplexing (OFDM) research. The amelioration of powerful multicarrier OFDM arrangements with multiple-input multiple-output (MIMO) systems has numerous benefits, which are detailed in this treatise. We continue by highlighting the limitations of conventional detection and channel estimation techniques designed for multiuser MIMO OFDM systems in the so-called rank-deficient scenarios, where the number of users supported or the number of transmit antennas employed exceeds the number of receiver antennas. This is often encountered in practice, unless we limit the number of users granted access in the base station's or radio port's coverage area. Following a historical perspective on the associated design problems and their state-of-the-art solutions, the second half of this treatise details a range of classic multiuser detectors (MUDs) designed for MIMO-OFDM systems and characterizes their achievable performance. A further section aims for identifying novel cutting-edge genetic algorithm (GA)-aided detector solutions, which have found numerous applications in wireless communications in recent years. In an effort to stimulate the cross pollination of ideas across the machine learning, optimization, signal processing, and wireless communications research communities, we will review the broadly applicable principles of various GA-assisted optimization techniques, which were recently proposed also

\footnotetext{
Manuscript received December 15, 2006; revised April 2, 2007. M. Jiang is with Samsung Electronics Research Institute, TW18 4QE Staines, U.K. (e-mail: ming.jiang@samsung.com).

L. Hanzo is with the School of Electrical and Computer Science (ECS), University of Southampton, SO17 1BJ Southampton, U.K. (e-mail: Ih@ecs.soton.ac.uk; http://www-mobile.ecs.soton.ac.uk).

Digital Object Identifier: 10.1109/JPROC.2007.898869
}

for employment in multiuser MIMO OFDM. In order to stimulate new research, we demonstrate that the family of GA-aided MUDs is capable of achieving a near-optimum performance at the cost of a significantly lower computational complexity than that imposed by their optimum maximum-likelihood (ML) MUD aided counterparts. The paper is concluded by outlining a range of future research options that may find their way into next-generation wireless systems.

KEYWORDS | Channel estimation; genetic algorithm (GA); multiple-input multiple-output (MIMO); multiuser detection/ detector (MUD); orthogonal frequency division multiplexing (OFDM); space division multiple access (SDMA)

\section{MOTIVATION AND INTRODUCTION TO MULTIPLE-INPUT MULTIPLE- OUTPUT (MIMO)-ORTHOGONAL FREQUENCY DIVISION MULTIPLEXING (OFDM) SYSTEMS}

During the past decades, wireless communication has benefitted from substantial advances and it is considered as the key enabling technique of innovative future consumer products. For the sake of satisfying the requirements of various applications, significant technological achievements are required to ensure that wireless devices have appropriate architectures suitable for supporting a wide range of services delivered to the users.

In the foreseeable future, the large-scale deployment of wireless devices and the requirements of highbandwidth applications are expected to lead to tremendous new challenges in terms of the efficient exploitation 
of the achievable spectral resources. Among the existing air-interface techniques, orthogonal frequency division multiplexing (OFDM) [1]-[4] has shown a number of advantages and has attracted substantial interest. New wireless techniques, such as ultra wideband (UWB) [5], advanced source and channel encoding as well as various smart antenna techniques, for example space-time codes (STCs) [6], space division multiple access (SDMA) [1] and beamforming, as well as other multiple-input multipleoutput (MIMO) [7], [8] wireless architectures are capable of offering substantial gains. Hence, researchers have focused their attention on the next generation of wireless broadband communications systems, which aim for delivering multimedia services requiring data rates beyond 2 Mbps. Undoubtedly, the support of such high data rates, while maintaining a high robustness against radio channel impairments requires further enhanced system architectures, which should aim for approaching the capacity of MIMO-aided systems communicating over the fading channels exemplified in Fig. 1 in the context of one or two transmit and one, two as well as six receivers, respectively. In a conceptually appealing, but somewhat simplistic manner, we may argue that the one-transmitter (1Tx) and one-receiver (1Rx) scenario is exposed to fading, since the vectorial sum of the multiple propagation paths may add constructively or destructively. By contrast, in case of the 2Tx and 6Rx scenario, for example, the chances are that at least one of the independently faded diversity-links benefits from the constructive interference of the received paths. To elaborate a little further, the achievable MIMO capacity [9] is exemplified for the specific scenario of 16-level quadrature amplitude modulation (QAM) transmissions over a twotransmitter, two-receiver MIMO system in Fig. 2. It becomes explicit in Fig. 2 that a MIMO system designed for achieving the maximum diversity gain-i.e. robustness against transmission errors-requires a lower channel signal-to-noise-ratio (SNR) than its counterpart dedicated to attaining the maximum multiplexing gain, i.e., throughput, but naturally, the lower operating SNR is maintained at the cost of a lower bit/symbol throughput. The variable $D$ in Fig. 2 indicates the number of dimensions exploited by the modulation scheme and $D=2$ corresponds to classic twodimensional QAM schemes.

In recent years various smart antenna designs have emerged, which have found application in diverse scenarios and the four most wide-spread MIMO types are briefly summarized in Table 1. These four MIMO schemes were designed for achieving various design goals. The family of Spatial Division Multiplexing (SDM) [1], [10] schemes aims for maximizing the attainable multiplexing gain, i.e., the throughput of a single user by exploiting the unique, antenna-specific channel impulse responses (CIRs) of the array elements. By contrast, SDMA arrangements [1] are close relatives of SDM schemes, but they maximise the number of users supported, as opposed to maximizing the throughput of a single user by sharing the total system
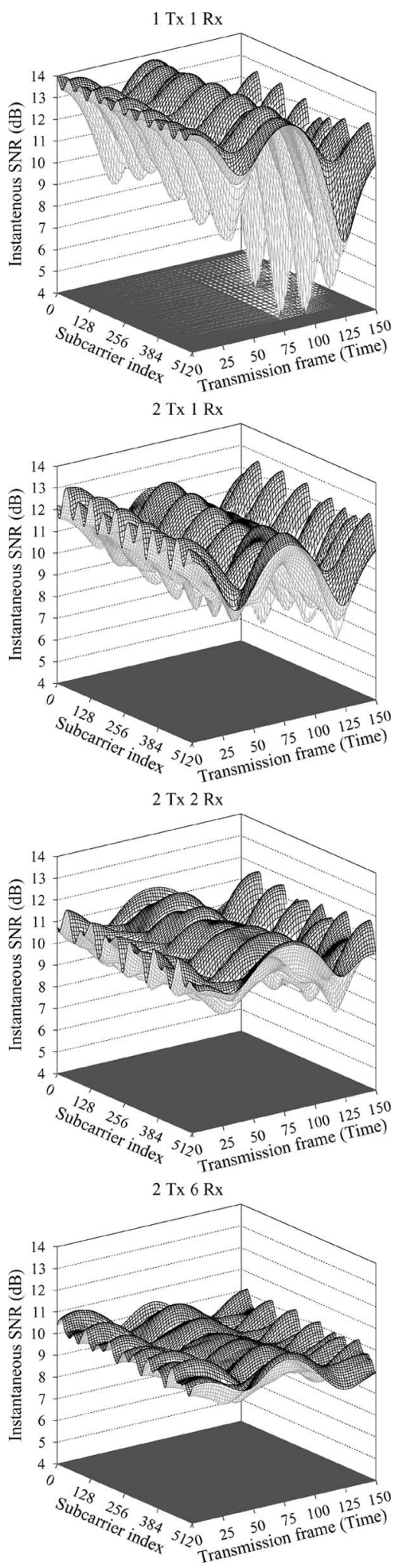

Fig. 1. Instantaneous channel SNR versus both time and frequency for a 512-subcarrier OFDM modem in the context of a single-transmitter single-receiver as well as for MIMO-aided two-transmitter systems using one, two and six receivers when communicating over an indoor wireless channel. The average channel SNR is $10 \mathrm{~dB}$ [6]. 


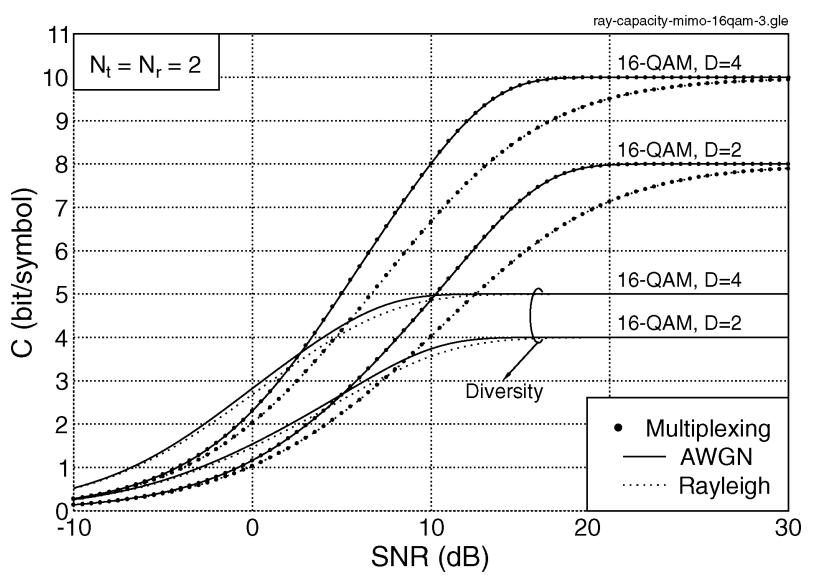

Fig. 2. The capacity of the MIMO uncorrelated Rayleigh-fading channel and additive white gaussian noise (AWGN) channel for classic 16 QAM $(M=16, D=2)$ and for so-called four-dimensional signalling $(M=32, D=4)$ [9].

throughput amongst the users supported. Alternatively, attaining the maximum possible diversity gain is the objective of the family of space-time block coding (STBC) [11] as well as space-time trellis coding (STTC) [12] schemes found in the literature [6]. In Fig. 1 the beneficial effects of second-order transmit and up to sixth-order receiver diversity was demonstrated in the context of STBC-aided MIMO-OFDM [13], [14], but space-time coding MIMOs will not be considered further in this treatise. Finally, beamforming mitigates the effects of interfering users roaming in the vicinity of the desired user [15], provided that their received signals are angularly separable, as demonstrated in Fig. 3. Similarly to spacetime coding, beamforming MIMOs will not be detailed further in this treatise.

We commence by briefly introducing the basic concept of OFDM, as a means of dealing with the problems of the so-called frequency selective fading exemplified in Fig. 1, when transmitting at a high rate, where the delayed and

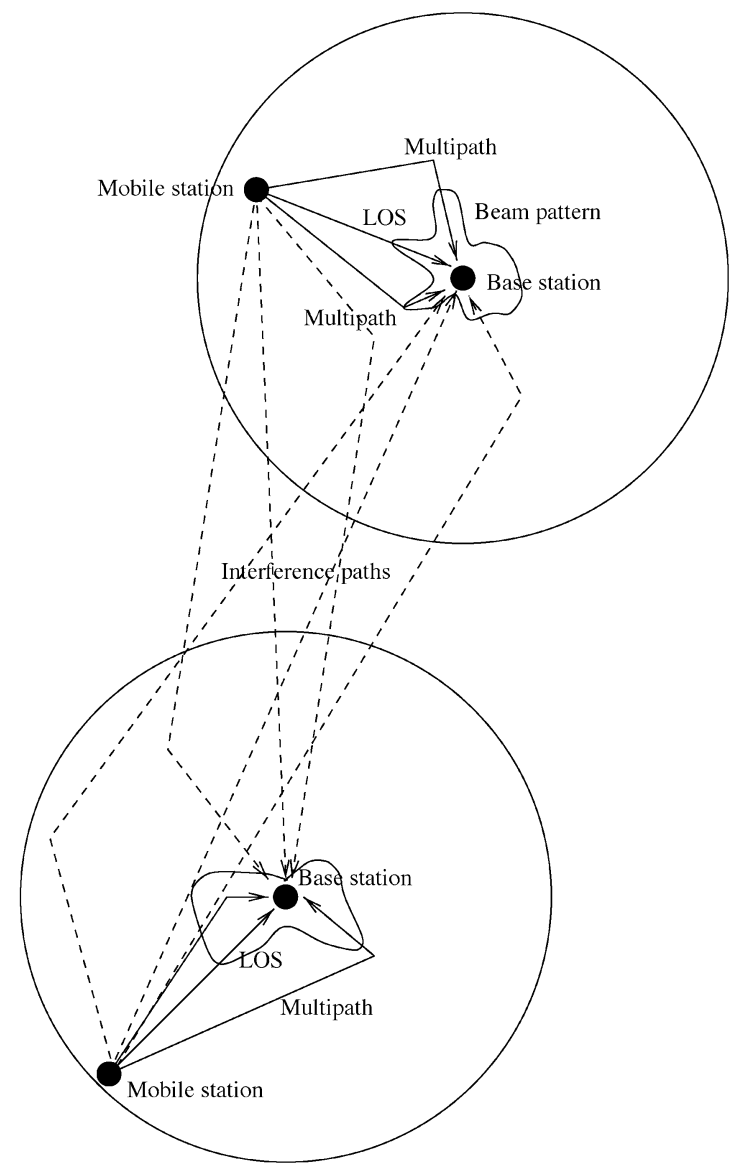

Fig. 3. The multipath environments of an uplink scenario, protraying the individual multipath components of the desired signals, the line-of-sight interference and the associated base station antenna array beam patterns [15].

reflected radio paths impose intersymbol interference (ISI) on the neighboring bits. The fundamental principle of OFDM originates from Chang [16], and over the years a multiplicity of researchers have investigated this

Table 1 The Four Main Applications of MIMOs in Wireless Communications

\begin{tabular}{|l|l|}
\hline $\begin{array}{l}\text { Space Division Multiplex- } \\
\text { ing (SDM) Systems [10] }\end{array}$ & $\begin{array}{l}\text { SDM systems employ multiple antennas, but in contrast to SDMA arrangements, not for the sake of supporting } \\
\text { multiple users. Instead, they aim for increasing the throughput of a wireless system in terms of the number of bits } \\
\text { per symbol that can be transmitted by a given user in a given bandwidth at a given integrity. }\end{array}$ \\
\hline $\begin{array}{l}\text { Space Division Multiple } \\
\text { Access (SDMA) [1], [21] }\end{array}$ & $\begin{array}{l}\text { SDMA exploits the unique, user-specific "spatial signature", i.e. the CIRs of the individual users for differentiating } \\
\text { amongst them. This allows the system to support multiple users within the same frequency band and/or time slot, } \\
\text { provided that their CIRs are sufficiently different and are accurately measured. SDMA/SDM systems constitute that } \\
\text { main focus of this contribution. }\end{array}$ \\
\hline $\begin{array}{l}\text { Spatial Diversity } \\
\text { (STBC [11], [14], [22], }\end{array}$ & $\begin{array}{l}\text { In contrast to the } \lambda / 2 \text {-spaced phased array elements of beamforming, in spatial diversity schemes, such as space-time } \\
\text { block or trellis codes [6] the multiple antennas are positioned as far apart as possible, so that the transmitted signals } \\
\text { STTC [6], [12], [14], the different antennas experience independent fading, resulting in the maximum achievable diversity gain. } \\
\text { [23], [24]) and Spac- } \\
\text { Time Spreading (STS) } \\
{[25],[26]}\end{array}$ \\
\hline Beamforming [15] & $\begin{array}{l}\text { Typically } \lambda / 2 \text {-spaced antenna elements are used for the sake of creating a spatially selective transmitter/receiver beam. } \\
\text { Smart antennas using beamforming have been employed for mitigating the effects of cochannel interfering signals and } \\
\text { for providing beamforming gain. }\end{array}$ \\
\hline
\end{tabular}


technique, as detailed in this paper. Despite its conceptual elegance, during its infancy the employment of OFDM has been mostly limited to military applications due to implementational difficulties. However, it has recently been adopted as the Digital Audio Broadcasting (DAB) [17] standard as well as the Digital Video Broadcasting (DVB) [18], [19] and for a range of other high-rate applications, such as wireless local area networks (WLANs) [20], as detailed below. These wide-ranging applications underline its significance as an alternative technique to conventional channel equalization [2] in order to combat signal dispersion.

In the OFDM scheme of Fig. 4 the serial data stream of a traffic channel is passed through a serial-to-parallel convertor, which splits the data-stream into $K$ number of low-rate parallel subchannels. The data symbols of each subchannel are applied to a modulator, where there are $K$ modulators whose carrier frequencies are $f_{0}, f_{1}, \ldots, f_{K}$. The difference between adjacent channels is $\Delta f$ and the overall bandwidth $W$ of the $K$ modulated carriers is $K \Delta f$. The substantial benefit of this approach is that the symbol duration of each of the $K$ subchannels is extended by a factor of $K$, where $K \leq 1024$ may be assumed and, hence, typically each subcarrier's signal is likely to remain unaffected by the multipath propagation. Hence, we can dispense with classic channel equalization [2]. These $K$ modulated carriers are then combined to generate the OFDM signal. We may view the serial-to-parallel (SP) convertor, as applying every Kth symbol to a modulator. This has the effect of interleaving the symbols forwarded to each modulator; hence, symbols $s_{0}, s_{K}, s_{2 K}, \ldots$, are applied to the modulator whose carrier frequency is $f_{0}$. At the receiver the received OFDM signal is demultiplexed into $K$ frequency bands, and the $K$ modulated signals are demodulated. The baseband signals are then recombined using a parallel-to-serial (PS) convertor.

Again, the main advantage of the above OFDM concept is that because the symbol period has been increased, the channel's delay spread becomes a significantly shorter fraction of a symbol period than in the serial system, potentially rendering the system less sensitive to ISI than the conventional serial system. In other words, in the lowrate subchannels the signal is no longer subject to frequency-selective fading, hence, channel equalization may be avoided.

At first sight it may appear to be a disadvantage of the OFDM approach shown in Fig. 4 that an increased complexity is imposed in comparison to a conventional serial modem, since we are employing $K$ modulators and "square-root-Nyquist" filters [2] at the transmitter and $K$ demodulators and "square-root-Nyquist" filters at the receiver. However, each subchannel modulator is operated at a $\mathrm{K}$-times lower symbol-rate and, hence, the system may be viewed as a "parallelized low-speed implementation" of a high-speed serial modem. As a further complexity mitigation technique, it may demonstrated mathematically [2] that this complexity can be further reduced by employing a fast Fourier transform (FFT)-based implementation, where a block of $K$ subchannels' signal is modulated onto the subcarriers in a single step. This is indeed quite plausible, since the $e^{j k f_{0} t}(k=1, \cdots, K)$ FFTkernels correspond to the subchannel modulators of Fig. 4. The subchannel modems can use arbitrary modulation schemes and in recent years high-throughput QAM

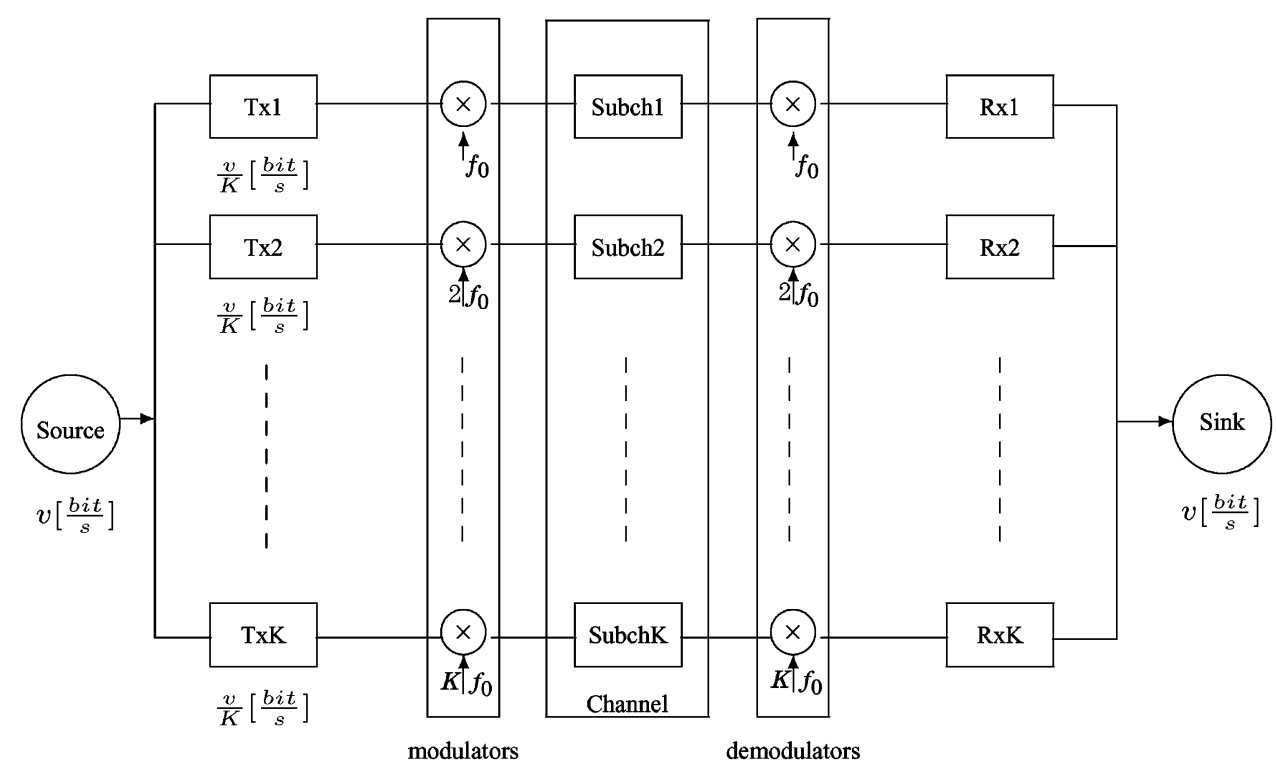

Fig. 4. Simplified block diagram of the orthogonal parallel modem. 
schemes have been favored. For a deeper tutorial exposure the interested reader is referred to [1], [2].

The organization of this paper is as follows. The portrayal of OFDM and its various applications from a historical perspective is provided in Section II. More specifically, Section II-A summarizes both the various international standards based on OFDM and the main research contributions to the OFDM literature, followed by a brief outline of MIMO-aided OFDM systems in Section II-B. Furthermore, in Sections III-A and B we point out the specific limitations of existing detection techniques and channel estimation approaches designed for multiuser MIMO OFDM systems, respectively. As a powerful tool proposed for finding near-optimum solutions to complex nonlinear optimization problems, genetic algorithms (GAs) [27]-[31] that were originally advocated by the evolutionary computing community have recently also been successfully exploited by the wireless communication community, bridging the interdisciplinary gap between the two historically distinct research communities. Aiming for providing efficient solutions to the problems stated in Section III, we highlight various GA-assisted techniques designed for multiuser MIMO OFDM in Sections IV-VI where numerous application examples are provided. Finally, our conclusions and a range of future research options are offered in Section VII.

\section{HISTORIC BACKGROUND}

\section{A. Orthogonal Frequency Division Multiplexing}

In recent years OFDM [1]-[4] has emerged as a promising air-interface technique. In the context of wired environments, OFDM techniques are also known as Discrete MultiTone (DMT) [32] transmissions and are employed in the American National Standards Institute's (ANSI) Asymmetric Digital Subscriber Line (ADSL) [33], High-bit-rate Digital Subscriber Line (HDSL) [34], and Very-high-speed Digital Subscriber Line (VDSL) [35] standards as well as in the European Telecommunication Standard Institute's (ETSI) [36] VDSL applications. In wireless scenarios, OFDM has been advocated by many European standards, such as Digital Audio Broadcasting (DAB) [17], Digital Video Broadcasting for Terrestrial Television (DVB-T) [18], Digital Video Broadcasting for Handheld Terminals (DVB-H) [19], Wireless Local Area Networks (WLANs) [20], and Broadband Radio Access Networks (BRANs) [37]. Furthermore, OFDM has been ratified as a standard or has been considered as a candidate standard by a number of standardization groups of the Institute of Electrical and Electronics Engineers (IEEE), such as the following.

- IEEE 802.11a [38]: An extension to IEEE 802.11 [39] that applies to WLANs and provides a bitrate of up to $54 \mathrm{Mbps}$ in the $5 \mathrm{GHz}$ band. In comparison to IEEE 802.11, where frequency-hopping spread spectrum (FHSS) or direct-sequence spread spectrum (DSSS) are used, IEEE 802.11a employs an OFDM scheme which applies to wireless asynchronous transfer mode (WATM) networks and access hubs.

- IEEE 802.11g [40]: Offers wireless transmission over relatively short distances at 20-54 Mbps in the $2.4 \mathrm{GHz}$ band. It also uses an OFDM scheme.

- IEEE 802.11n [41]: Candidate standard for next generation WLANs, which was created from previous IEEE 802.11 standards by incorporating MIMO techniques. It offers high-throughput wireless transmission at 100-200 Mbps.

- IEEE 802.16 [42]: Defines wireless services operating in the $2-11 \mathrm{GHz}$ band associated with wireless metropolitan area networks (WMANs), providing a communication link between a subscriber and a core network, e.g., the public telephone network and the Internet.

The first OFDM schemes date back to the 1960s, which were proposed by Chang [16] and Saltzberg [43]. In the classic parallel data transmission systems [16], [43], the frequency-domain (FD) bandwidth is divided into a number of nonoverlapping subchannels, each of which hosts a specific carrier widely referred to as a subcarrier. While each subcarrier is separately modulated by a data symbol, the overall modulation operation across all the subchannels results in a frequency-multiplexed signal. Since the modulated signal's spectrum is multiplied by a rectangular window corresponding to the length of the time-domain (TD) OFDM symbol, the subcarriers have to be convolved with resultant FD sinc-function. Similarly to classic ISI-free orthogonal TD Nyquist-signalling, all of the sinc-shaped FD subchannel spectra exhibit zero-crossings at all of the surrounding subcarrier frequencies and, hence, the individual subchannel spectra are orthogonal to each other. This ensures that the subcarrier signals do not interfere with each other, when communicating over perfectly distortionless channels, as a consequence of their orthogonality [1].

The early OFDM schemes [16], [43], [44], [51] required banks of sinusoidal subcarrier generators and demodulators, which imposed a high implementation complexity. This drawback limited the application of OFDM to military systems until 1971, when Weinstein and Ebert [45] suggested that the discrete Fourier transform (DFT) can be used for the OFDM modulation and demodulation processes, which significantly reduces the implementation complexity of OFDM. Since then, more practical OFDM research has been carried out. For example, in the early 1980s Peled and Ruiz [52] proposed a simplified FD data transmission method using a cyclic prefix aided technique and exploited reducedcomplexity algorithms for achieving a significantly lower computational complexity than that of classic 
single-carrier time-domain QAM [2] modems. Around the same era, Keasler et al. [47] invented a high-speed OFDM modem for employment in switched networks, such as the telephone network. Hirosaki designed a subchannelbased equalizer for an orthogonally multiplexed QAM system in 1980 [46] and later introduced the DFT-based implementation of OFDM systems [53], based on which a so-called groupband data modem was developed [54]. Cimini, Jr. [48] and Kalet [55] investigated the performance of OFDM modems in mobile communication channels. Furthermore, Alard and Lassalle [49] applied OFDM in digital broadcasting systems, which was the pioneering work of the European DAB standard [17] established in the mid-1990s. More recent advances in OFDM transmission were summarized in the state-of-theart books [1], [3], [56] as well as a number of overview papers [4], [57]-[60].

OFDM has some key advantages over other widely used wireless access techniques, such as time division multiple access (TDMA) [61], frequency division multiple access (FDMA) [61], and code division multiple access (CDMA) [25], [62]-[65]. The main merit of OFDM is the fact that the radio channel is divided into many narrowband, low-rate, frequency-nonselective subchannels or subcarriers, so that multiple symbols can be transmitted in parallel, while maintaining a high spectral efficiency. Each subcarrier may also deliver information for a different user, resulting in a simple multiple access scheme known as orthogonal frequency division multiple access (OFDMA) [66]-[69]. This enables different media such as video, graphics, speech, text, or other data to be transmitted within the same radio link, depending on the specific types of services and their quality-of-service (QoS) requirements. Furthermore, in OFDM systems different modulation schemes can be employed for different subcarriers or even for different users. For example, the users close to the base station (BS) may have a relatively good channel quality; thus, they can use high-order modulation schemes to increase their data rates. By contrast, for those users that are far from the BS or are serviced in highly loaded urban areas, where the subcarriers' quality is expected to be poor, low-order modulation schemes can be invoked [70].

Besides its implementational flexibility, the low complexity required in transmission and reception as well as the attainable high performance render OFDM a highly attractive candidate for high data-rate communications over time-varying frequency-selective radio channels. Incorporating channel coding techniques into OFDM systems, which results in Coded OFDM (COFDM) [71], [72], allows us to maintain robustness against frequency-selective fading channels, where busty errors are encountered at specific subcarriers in the FD. Additionally, when using a cyclic prefix [52], OFDM exhibits a high resilience against the ISI introduced by multipath propagation.
As a summary of this section, we outline the milestones and the main contributions found in the OFDM literature in Tables 2-4.

\section{B. Multiple-Input Multiple-Output Assisted OFDM}

1) The Benefits of MIMOs: High data-rate wireless communications have attracted significant interest and constitute a substantial research challenge in the context of the emerging WLANs and other indoor multimedia networks. Specifically, the employment of multiple antennas at both the transmitter and the receiver, which is widely referred to as the MIMO technique, constitutes a cost-effective approach to high-throughput wireless communications.

The concepts of MIMOs have been under development for many years for both wired and wireless systems. The earliest MIMO applications in wireless communications date back to the mid-1980s, when Winters [7], [8], [107], [108] published a number of breakthrough contributions, where he introduced a technique of transmitting data from multiple users over the same frequency/ time channel using multiple antennas at both the transmitter and receiver ends. Sparked off by Winters' pioneering work [7], Salz [109] investigated joint transmitter/ receiver optimization using the minimum mean square error (MMSE) criterion. Since then, Winters and others [110]-[116] have made further significant advances in the field of MIMOs. In 1996, Raleigh and Cioffi [117] and Foschini [10] proposed new approaches for improving the efficiency of MIMO systems, which inspired numerous further contributions [118]-[126].

As a key building block of next-generation wireless communication systems, MIMOs are capable of supporting significantly higher data rates than the universal mobile telecommunications system (UMTS) and the high-speed downlink packet access (HSDPA)-based 3G networks [127]. As indicated by the terminology, a MIMO system employs multiple transmitter and receiver antennas for delivering parallel data streams, as illustrated in Fig. 5. Since the information is transmitted through different paths, a MIMO system is capable of exploiting both transmitter and receiver diversity, hence maintaining reliable communications. Furthermore, with the advent of multiple antennas, it becomes possible to jointly process/combine the multiantenna signals and, thus, improves the system's integrity and/or throughput. Briefly, compared to single-input single-output (SISO) systems, the two most significant advantages of MIMO systems are as follows.

- A significant increase of both the system's capacity and spectral efficiency. The capacity of a wireless link increases linearly with the minimum of the number of transmitter or the receiver antennas [117], [118]. The data rate can be increased by spatial multiplexing without consuming more 
Table 2 Milestones in the History of OFDM

\begin{tabular}{|c|c|}
\hline Year & Milestone \\
\hline 1966 & First OFDM scheme proposed by Chang [16] for dispersive fading channels. \\
\hline 1967 & Saltzberg [43] studied a multi-carrier system employing Orthogonal QAM (O-QAM) of the carriers. \\
\hline 1970 & U.S. patent on OFDM issued [44]. \\
\hline 1971 & Weinstein and Ebert [45] applied DFT to OFDM modems. \\
\hline \multirow[t]{2}{*}{1980} & Hirosaki designed a subchannel-based equalizer for an orthogonally multiplexed QAM system [46]. \\
\hline & Keasler et al. [47] described an OFDM modem for telephone networks. \\
\hline 1985 & Cimini [48] investigated the feasibility of OFDM in mobile communications. \\
\hline 1987 & Alard and Lasalle [49] employed OFDM for digital broadcasting. \\
\hline 1991 & ANSI ADSL standard [33]. \\
\hline 1994 & ANSI HDSL standard [34]. \\
\hline 1995 & ETSI DAB standard [17]: the first OFDM-based standard for digital broadcasting systems. \\
\hline 1996 & ETSI WLAN standard [20]. \\
\hline 1997 & ETSI DVB-T standard [18]. \\
\hline \multirow[t]{2}{*}{1998} & ANSI VDSL and ETSI VDSL standards [35], [36]. \\
\hline & ETSI BRAN standard [37]. \\
\hline 1999 & IEEE 802.11a WLAN standard [38]. \\
\hline 2002 & IEEE $802.11 \mathrm{~g}$ WLAN standard [40]. \\
\hline \multirow{3}{*}{2004} & ETSI DVB-H standard [19]. \\
\hline & IEEE 802.16 WMAN standard [42]. \\
\hline & Candidate for IEEE $802.11 \mathrm{n}$ standard for next generation WLAN [41]. \\
\hline 2005 & Candidate for 4G standards in China, Japan and South Korea (CJK) [50]. \\
\hline
\end{tabular}

frequency resources and without increasing the total transmit power.

- Dramatic reduction of the effects of fading due to the increased diversity. This is particularly beneficial, when the different channels fade independently.

A comprehensive overview of MIMO techniques covering channel models, performance limits, coding and transceiver designs can be found in [128].

2) MIMO OFDM: The quality of a wireless link can be described by three basic parameters, namely the transmission rate, the transmission range and the transmission reliability. Conventionally, the transmission rate may be increased by reducing the transmission range and reliability. By contrast, the transmission range may be extended at the cost of a lower transmission rate and reliability, while the transmission reliability may be improved by reducing the transmission rate and range [129]. However, with the advent of MIMO assisted OFDM systems, the above-mentioned three parameters may be simultaneously improved [129]. Initial field tests of broadband wireless MIMO OFDM communication systems have shown that an increased capacity, coverage and reliability is achievable with the aid of MIMO techniques [130]. Furthermore, although MIMOs can potentially be combined with any modulation or multiple access technique, recent research suggests that the implementation of MIMO-aided OFDM is more efficient, as a benefit of the straightforward matrix algebra invoked for processing the MIMO OFDM signals [129].

MIMO OFDM, which is claimed to be invented by Airgo Networks [131], has formed the foundation of all candidate standards proposed for IEEE 802.11n [41]. In recent years, this topic has attracted substantial research efforts, addressing numerous aspects, such as system capacity [132], [133], space/time/frequency coding [134][138], peak-to-average power ratio (PAPR) control [139]-[141], channel estimation [142]-[144], receiver design [145]-[148], etc. Recently, Paulraj et al. [128] and Stüber et al. [149] provided compelling overviews of MIMO OFDM communications. Furthermore, Nortel Networks has developed a MIMO OFDM prototype [150] during late 2004, which demonstrates the superiority of MIMO OFDM over today's networks in terms of the achievable data rate. For the reader's convenience, we have summarized the major contributions on MIMO OFDM in Tables 5, 6, and 7 at a glance. 
3) SDMA-Based MIMO OFDM Systems: As a subclass of MIMO arrangements, recently the SDMA [1], [202]-[204] based techniques have attracted substantial interest. As one of the most promising techniques aiming at solving the capacity problem of wireless communication systems, SDMA enables multiple users to simultaneously share the same bandwidth in different geographical locations. More specifically, the exploitation of the spatial dimension, namely the so-called spatial signature, makes it possible to identify the individual users, even when they are in the same time/frequency/code domains, thus increasing the system's capacity.

In Fig. 6 we illustrate the concept of SDMA systems. As shown in Fig. 6, each user exploiting a single transmitter antenna aided mobile station (MS) simultaneously communicates with the BS equipped with an array of receiver antennas. Explicitly, SDMA can be considered as a specific branch of the family of MIMO systems, where the

Table 3 Main Contributions on OFDM (Part 1)

\begin{tabular}{|c|c|c|}
\hline Year & Author(s) & Contribution \\
\hline 1966 & Chang [16] & Proposed the first OFDM scheme. \\
\hline 1967 & Saltzberg [43] & Studied a multi-carrier system employing O-QAM. \\
\hline 1968 & Chang and Gibby [51] & $\begin{array}{l}\text { Presented a theoretical analysis of the performance of an orthogonal multiplexing data transmission } \\
\text { scheme. }\end{array}$ \\
\hline 1970 & Chang [44] & U.S. patent on OFDM issued. \\
\hline 1971 & Weinstein and Ebert [45] & Applied DFT to OFDM modems. \\
\hline \multirow{3}{*}{1980} & Hirosaki [46] & Designed a subchannel-based equalizer for an orthogonally multiplexed QAM system. \\
\hline & Peled and Ruiz [52] & $\begin{array}{l}\text { Described a reduced-complexity FD data transmission method together with a cyclic prefix } \\
\text { technique. }\end{array}$ \\
\hline & Keasler et al. [47] & Invented an OFDM modem for telephone networks. \\
\hline 1981 & Hirosaki [53] & Suggested a DFT-based implementation of OFDM systems. \\
\hline 1985 & Cimini [48] & Investigated the feasibility of OFDM in mobile communications. \\
\hline 1986 & $\begin{array}{l}\text { Hirosaki, Hasegawa and } \\
\text { Sabato [54] }\end{array}$ & Developed a groupband data modem using an orthogonally multiplexed QAM technique. \\
\hline 1987 & Alard and Lasalle [49] & Employed OFDM for digital broadcasting. \\
\hline 1989 & Kalet [55] & Analyzed multitone QAM modems in linear channels. \\
\hline 1990 & Bingham [4] & Discussed various aspects of early OFDM techniques in depth. \\
\hline 1991 & Cioffi [33] & Introduced the ANSI ADSL standard. \\
\hline $\begin{array}{l}1993- \\
1995\end{array}$ & $\begin{array}{l}\text { Warner [73], Moose [74] } \\
\text { and Pollet [75] }\end{array}$ & Conducted studies on time and frequency synchronization in OFDM systems. \\
\hline $\begin{array}{l}1994- \\
1996\end{array}$ & $\begin{array}{l}\text { Jones [76], Shepherd [77] } \\
\text { and Wulich [78], [79] }\end{array}$ & $\begin{array}{l}\text { Explored various coding and post-processing techniques designed for minimizing the peak power } \\
\text { of the OFDM signal. }\end{array}$ \\
\hline \multirow[t]{2}{*}{1997} & Li and Cimini [80], [81] & Revealed how clipping and filtering affect OFDM systems. \\
\hline & Hara and Prasad [82] & Compared various methods of combining CDMA and OFDM. \\
\hline \multirow[b]{2}{*}{1998} & $\begin{array}{l}\text { Li, Cimini and Sollen- } \\
\text { berger [83] }\end{array}$ & $\begin{array}{l}\text { Designed a robust Minimum Mean Square Error (MMSE) based channel estimator for OFDM } \\
\text { systems. }\end{array}$ \\
\hline & $\begin{array}{l}\text { May, Rohling and En- } \\
\text { gels [71] }\end{array}$ & $\begin{array}{l}\text { Carried out a performance analysis of Viterbi decoding in the context of 64-Differential Amplitude } \\
\text { and Phase-Shift Keying (64-DAPSK) and 64QAM modulated OFDM signals. }\end{array}$ \\
\hline \multirow{5}{*}{1999} & Li and Sollenberger [84] & $\begin{array}{l}\text { Focused on parameter estimation invoked by a MMSE diversity combiner designed for adaptive } \\
\text { antenna array aided OFDM. }\end{array}$ \\
\hline & $\begin{array}{l}\text { Armour, Nix and Bull } \\
{[85]-[87]}\end{array}$ & $\begin{array}{l}\text { Illustrated the combined OFDM-equalization aided receiver and the design of pre-Fast Fourier } \\
\text { Transform (FFT) equalizers. }\end{array}$ \\
\hline & $\begin{array}{l}\text { Prasetyo and Aghvami } \\
{[88],[89]}\end{array}$ & $\begin{array}{l}\text { Simplified the transmission frame structure for achieving fast burst synchronization in OFDM } \\
\text { systems. }\end{array}$ \\
\hline & $\begin{array}{l}\text { Cherriman, Keller and } \\
\text { Hanzo [90] }\end{array}$ & $\begin{array}{l}\text { Evaluated a packetization and packet acknowledgment scheme assisted H. } 263 \text { video codec in OFDM } \\
\text { scenarios. }\end{array}$ \\
\hline & Wong et al. [91] & $\begin{array}{l}\text { Advocated a subcarrier, bit and power allocation algorithm to minimize the total transmit power } \\
\text { of multi-user OFDM. }\end{array}$ \\
\hline
\end{tabular}


Table 4 Main Contributions on OFDM (Part 2)

\begin{tabular}{|c|c|c|}
\hline Year & Author(s) & Contribution \\
\hline \multirow{6}{*}{2000} & Fazel and Fettweis [92] & A collection of state-of-the-art works on OFDM. \\
\hline & Van Nee and Prasad [3] & OFDM for wireless multimedia communications. \\
\hline & $\begin{array}{l}\text { Lee, Keller and Hanzo } \\
\text { [93] }\end{array}$ & Studied enhanced turbo-coded OFDM receivers designed for DVB-T systems. \\
\hline & Keller and Hanzo [58] & Highlighted the adaptive bit allocation and turbo coding schemes in the context of OFDM. \\
\hline & $\begin{array}{l}\text { Lin, Cimini and Chuang } \\
\text { [72] }\end{array}$ & Invoked turbo coding in an OFDM system using diversity. \\
\hline & Keller and Hanzo [94] & Analyzed the design tradeoffs of turbo-coded burst-by-burst adaptive OFDM wideband transceivers. \\
\hline \multirow{3}{*}{$\begin{array}{l}2001- \\
2002\end{array}$} & Keller et al. [95] & $\begin{array}{l}\text { Quantified the effect of time-domain and frequency-domain synchronization errors in OFDM } \\
\text { systems. }\end{array}$ \\
\hline & Lu and Wang [96]-[99] & Considered channel coded STC-assisted OFDM systems. \\
\hline & $\begin{array}{l}\text { Cherriman, Keller and } \\
\text { Hanzo [100] }\end{array}$ & $\begin{array}{l}\text { A range of adaptive OFDM video systems were proposed for interactive communications over } \\
\text { wireless channels. }\end{array}$ \\
\hline 2003 & $\begin{array}{l}\text { Hanzo, Münster, Choi } \\
\text { and Keller [1] }\end{array}$ & OFDM for broadband multi-user communications, WLANs and broadcasting. \\
\hline \multirow{5}{*}{2004} & $\begin{array}{l}\text { Simeone, Bar-Ness and } \\
\text { Spagnolini [101] }\end{array}$ & Demonstrated a subspace tracking algorithm used for channel estimation in OFDM systems. \\
\hline & $\begin{array}{l}\text { J. Zhang, Rohling and P. } \\
\text { Zhang [102] }\end{array}$ & $\begin{array}{l}\text { Adopted an Inter-Carrier Interference (ICI) cancellation scheme to combat the ICI in OFDM } \\
\text { systems. }\end{array}$ \\
\hline & Necker and Stüber [103] & $\begin{array}{l}\text { Exploited a blind channel estimation scheme based on the Maximum Likelihood (ML) principle } \\
\text { in OFDM systems. }\end{array}$ \\
\hline & Doufexi et al. [104] & Reflected the benefits of using sectorized antennas in WLANs. \\
\hline & $\begin{array}{l}\text { Alsusa, Lee and } \\
\text { McLaughlin [105] }\end{array}$ & Proposed packet based multi-user OFDM systems using adaptive subcarrier-user allocation. \\
\hline 2005 & Williams et al. [106] & Evaluated a pre-FFT synchronisation method for OFDM. \\
\hline
\end{tabular}

transmissions of the multiple transmitter antennas cannot be coordinated, simply because they belong to different users. Briefly speaking, the major advantages of SDMA techniques are as follows [205].

- Range extension: With the aid of antenna array, the coverage area of high-integrity reception can be significantly larger than that of any single-antenna aided systems. In a SDMA system, the number of

Transmit antennas

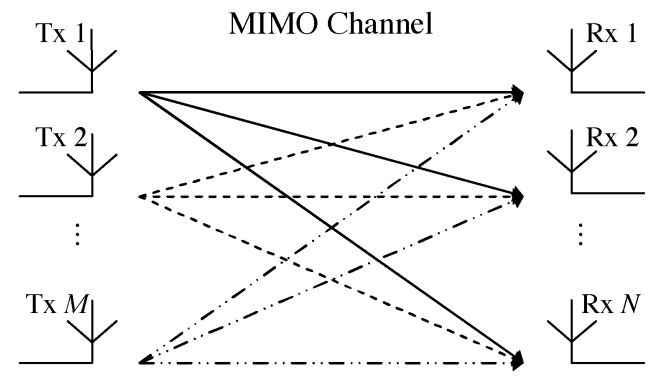

Fig. 5. Schematic of the generic MIMO system employing $M$ transmitter antennas and $N$ receiver antennas. cells required for covering a given geographic area can be substantially reduced. For example, a tenelement array offers a gain of ten, which typically doubles the radius of the cell and, hence, quadruples the coverage area.

- Multipath mitigation: Benefitting from the MIMO architecture, in SDMA systems the detrimental effects of multipath propagations are effectively mitigated. Furthermore, in specific scenarios the multipath phenomenon can even be exploited for enhancing the desired users' signals by employing efficient receiver diversity schemes.

- Capacity increase: Theoretically, SDMA can be incorporated into any existing multiple access standard at the cost of a limited increase in system complexity, while attaining a substantial increase in capacity. For instance, by applying SDMA to a conventional TDMA system, two or more users can share the same time slots, resulting in a doubled or higher overall system capacity.

- Interference suppression: The interference imposed by other systems and by users in other cells can be significantly reduced by exploiting the desired user's unique, user-specific CIRs. 
Table 5 Main Contributions on MIMO OFDM (Part 1)

\begin{tabular}{|c|c|c|}
\hline Year & Author(s) & Contribution \\
\hline \multirow[b]{2}{*}{2001} & Blum et al. [134] & Studied improved space-time coding techniques for MIMO OFDM systems. \\
\hline & Piechocki et al. [151] & $\begin{array}{l}\text { Reported on the performance of spatial multiplexing using ML decoding for a Vertical Bell Labs Layered Space- } \\
\text { Time (V-BLAST) OFDM system. }\end{array}$ \\
\hline \multirow{10}{*}{2002} & $\mathrm{Li}[142]$ & $\begin{array}{l}\text { Exploited optimum training sequence design and simplified channel estimation for improving the performance } \\
\text { and for reducing the complexity of channel parameter estimation in MIMO OFDM systems. }\end{array}$ \\
\hline & $\begin{array}{l}\text { Bolckei, Gesbert and } \\
\text { Paulraj [132] }\end{array}$ & $\begin{array}{l}\text { Analyzed the influence of physical parameters such as the amount of delay spread, cluster angle spread and total } \\
\text { angle spread, as well as system parameters such as the number of antennas and the antenna spacing on both the } \\
\text { ergodic capacity and outage capacity. }\end{array}$ \\
\hline & Chuah et al. [152] & Derived and compared the asymptotic growth rate of capacity in multiple-antenna aided systems. \\
\hline & Catreux et al. [153] & Offered an overview of the challenges and promises of link adaptation in future broadband wireless networks. \\
\hline & Piechocki et al. [154] & $\begin{array}{l}\text { Presented a performance evaluation of spatial multiplexing and space-frequency coded modulation schemes } \\
\text { designed for WLANs. }\end{array}$ \\
\hline & $\begin{array}{l}\text { Molisch, Win and Winters } \\
{[155]}\end{array}$ & Proposed a reduced-complexity method for grouping multiple antennas and space-time codes. \\
\hline & $\begin{array}{l}\text { Li, Winters and Sollen- } \\
\text { berger [145] }\end{array}$ & Invoked space-time coding and Successive Interference Cancellation (SIC) in MIMO OFDM systems. \\
\hline & Stamoulis et al. [156] & Revealed the effects of ICI on MIMO OFDM. \\
\hline & Doufexi et al. [157] & $\begin{array}{l}\text { Characterized the outdoor physical layer performance of a coded MIMO OFDM system using space-time } \\
\text { processing. }\end{array}$ \\
\hline & Giangaspero et al. [146] & Compared two Co-Channel Interference (CCI) cancellation schemes in the context of MIMO OFDM. \\
\hline \multirow{10}{*}{2003} & Li, Letaief and Cao [147] & $\begin{array}{l}\text { Advocated a CCI cancellation method using angle diversity based on null-steering or minimum variance distortion } \\
\text { response beamforming. }\end{array}$ \\
\hline & $\begin{array}{l}\text { Bölcskei, Borgmann and } \\
\text { Paulraj [158] }\end{array}$ & Measured the impact of the propagation environment on the performance of space-frequency coded MIMO OFDM. \\
\hline & $\begin{array}{l}\text { Barhumi, Leus and Moo- } \\
\text { nen [143] }\end{array}$ & $\begin{array}{l}\text { Described a Least-Squares (LS) channel estimation scheme designed for MIMO OFDM systems based on pilot } \\
\text { tones. }\end{array}$ \\
\hline & Ganesan and Sayeed [133] & $\begin{array}{l}\text { Derived a virtual MIMO framework for single-transmitter, single-receiver multipath fading channels that enables } \\
\text { maximal exploitation of channel diversity at both the transmitter and the receiver. }\end{array}$ \\
\hline & Gamal et al. [135] & $\begin{array}{l}\text { Utilized an OFDM technique to transform the MIMO multi-path channel into a MIMO flat block fading channel, } \\
\text { where the associated diversity is exploited by employing space-frequency codes. }\end{array}$ \\
\hline & Moon et al. [139] & $\begin{array}{l}\text { Evaluated the PAPR performance in a MIMO OFDM-based WLAN system using a Space-Time Block Code } \\
\text { (STBC). }\end{array}$ \\
\hline & Cai, Song and Li [159] & $\begin{array}{l}\text { Developed a technique based on the auto-correlation function for estimating the Doppler spread in Rayleigh fading } \\
\text { channels for mobile OFDM systems using multiple antennas. }\end{array}$ \\
\hline & Leus and Moonen [160] & Employed tone-by-tone based equalization techniques in MIMO OFDM systems. \\
\hline & Lee et al. [140] & Investigated the PAPR characteristics in a MIMO OFDM system using the selective mapping approach. \\
\hline & Piechocki et al. [161] & Devised a blind method for joint detection of space-time coded MIMO OFDM. \\
\hline
\end{tabular}

- Compatibility: SDMA is compatible with most of the existing modulation schemes, carrier frequencies and other specifications. Furthermore, it can be readily implemented using various array geometries and antenna types.

The combination of SDMA and OFDM results in SDMA-OFDM systems [1], [202], [206], [207], which exploit the merits of both SDMA and OFDM, having attracted more and more interest [207]-[213]. Tables 8 and 9 summarize the main contributions on SDMA and SDMA-OFDM found in the open literature.

\section{LIMITATION OF EXISTING DETECTION AND CHANNEL ESTIMATION TECHNIQUES DESIGNED FOR MULTIUSER MIMO OFDM}

Undoubtedly, MIMO OFDM has demonstrated a high potential for employment in future high rate wireless communication systems [128], [149]. However, the associated detection and channel estimation techniques found in the multiuser MIMO OFDM literature have various limitations.

\section{A. Multiuser Detection}

Fig. 7 illustrates the most popular multiuser detection (MUD) schemes proposed for MIMO OFDM systems throughout these years. Among the various MUDs, the classic linear least squares (LS) [1], [268] and MMSE [1], [146], [268] MUDs exhibit a low complexity at the cost of a limited performance. By contrast, the high-complexity optimum maximum-likelihood (ML) MUD [1], [202], [207], [268] is capable of achieving the best performance owing to invoking an exhaustive search, which imposes a computational complexity typically increasing exponentially with the number of simultaneous users supported by the MIMO OFDM system and, thus rendering its implementation prohibitive in high-user-load scenarios. 
In the literature, a range of suboptimal nonlinear MUDs have also been proposed, such as for example the MUDs based on successive interference cancellation (SIC) [1], [202], [268] or parallel interference cancellation (PIC) [1], [268] techniques. Explicitly, instead of detecting and demodulating the users' signals in a sequential manner, as the LS and MMSE MUDs do, the PIC and SIC MUDs invoke an iterative processing technique that combines detection and demodulation. More specifically, the output signal generated during the previous detection iteration is demodulated and fed back to the input of the MUD for the next iterative detection step. Similar techniques invoking decision-feedback have been applied also in the context of classic channel equalization. However, since the philosophy of both the PIC and SIC MUDs is based on the principle of removing the effects of the interfering users during each detection stage, they are prone to error propagation occurring during the consecutive detection stages due to the erroneously detected signals of the previous stages [1]. In order to mitigate the effects of error propagation, an attractive design alternative is to simultaneously detect all the users' signals, rather than invoking iterative interference cancellation schemes. Recently, another family of multiuser detection schemes referred to as sphere decoders (SDs) [269]-[275] as well as their derivatives such as the optimized hierarchy reduced search algorithm (OHRSA)aided MUD [276], [277], have also been proposed for multiuser systems, which are capable of achieving ML performance at a lower complexity. Other MUD techniques, for example those based on the minimum biterror rate (MBER) MUD algorithms [211], [212] have also been advocated.

As far as we are concerned, however, most of the above-mentioned techniques were proposed for the systems, where the number of users $L$ is less than or equal to the number of receivers $P$, referred to here as the underloaded or fully loaded scenarios, respectively. Nonetheless, in practical applications it is possible that $L$ exceeds $P$, which is often referred to as a rank-deficient scenario, where we have no control over the number of users roaming in the base station's coverage area. In rankdeficient systems the $(P \times L)$-dimensional MIMO channel matrix representing the $(P \times L)$ number of channel

Table 6 Main Contributions on MIMO OFDM (Part 2)

\begin{tabular}{|c|c|c|}
\hline Year & Author(s) & Contribution \\
\hline \multirow{20}{*}{2004} & $\begin{array}{l}\text { Shin, H. Lee and C. Lee } \\
\text { [144] }\end{array}$ & $\begin{array}{l}\text { Suggested a cyclic comb-type training structure for reducing the Mean-Square Errors (MSEs) at the edge } \\
\text { subcarriers of MIMO OFDM signals. }\end{array}$ \\
\hline & $\begin{array}{l}\text { Xia, Zhou and Giannakis } \\
\text { [162] }\end{array}$ & $\begin{array}{l}\text { Created an adaptive MIMO OFDM transmitter by applying an adaptive two-dimensional coder-beamformer with } \\
\text { the aid of partial channel knowledge. }\end{array}$ \\
\hline & Huang and Letaief [163] & Portrayed an OFDM symbol based space diversity technique. \\
\hline & Butler and Collings [164] & $\begin{array}{l}\text { Employed an approximate log-likelihood decoding approach based on a Zero-Forcing (ZF) receiver for bit- } \\
\text { interleaved coded modulation assisted MIMO OFDM systems. }\end{array}$ \\
\hline & Stüber et al. [149] & Summarized various physical layer research challenges in MIMO OFDM system design. \\
\hline & Paulraj et al. [128] & Provided an overview of MIMO and/or MIMO OFDM systems. \\
\hline & Lu, Yue and Wang [165] & $\begin{array}{l}\text { Identified the performance of an optimized MIMO OFDM scheme using Low Density Parity Check (LDPC) } \\
\text { codes. }\end{array}$ \\
\hline & $\begin{array}{l}\text { Van Zelst and Schenk } \\
{[166]}\end{array}$ & $\begin{array}{l}\text { Implemented MIMO OFDM processing and evaluated its performance by both simulations and experimental test } \\
\text { results. }\end{array}$ \\
\hline & $\begin{array}{l}\text { Pascual-Iserte, Pérez- } \\
\text { Neira and Lagunas [167] }\end{array}$ & $\begin{array}{l}\text { Conducted studies on maximizing the Signal to Noise and Interference Ratio (SNIR) over the subcarriers subject } \\
\text { to a total transmit power constraint. }\end{array}$ \\
\hline & Zeng and $\mathrm{Ng}[168]$ & $\begin{array}{l}\text { Contrived a subspace-based semi-blind method for estimating the channel responses of a multi-user and multi- } \\
\text { antenna OFDM uplink system. }\end{array}$ \\
\hline & Alien et al. [169] & Assessed the performance of spatial diversity in an OFDM WLAN for various antenna topologies. \\
\hline & $\begin{array}{l}\text { Dayal, Brehler and } \\
\text { Varanasi [136] }\end{array}$ & $\begin{array}{l}\text { Introduced space-time channel-sounding training codes designed for multiple-antenna, noncoherent, multiple block } \\
\text { Rayleigh fading channel. }\end{array}$ \\
\hline & Park and Kang [148] & $\begin{array}{l}\text { Adopted a reduced-complexity iterative algorithm for joint Maximum-A-Posteriori (MAP) detection and CCI } \\
\text { suppression in MIMO OFDM systems. }\end{array}$ \\
\hline & Tan and Stüber [170] & Combined cyclic delay diversity and MIMO OFDM for achieving full spatial diversity in flat-fading channels. \\
\hline & $\begin{array}{l}\text { Wang, Shayan and } \\
\text { Zeng [171] }\end{array}$ & $\begin{array}{l}\text { Illustrated the diversity and coding advantages in terms of the minimum Hamming distance and the minimum } \\
\text { squared product distance of the code as well as the relative frequencies. }\end{array}$ \\
\hline & Pan, Letaief and Cao [172] & $\begin{array}{l}\text { Discussed dynamic spatial subchannel allocation in conjunction with adaptive beamforming in broadband OFDM } \\
\text { wireless systems. }\end{array}$ \\
\hline & $\begin{array}{l}\text { Tepedelenlioĝlu and } \\
\text { Challagulla [173] }\end{array}$ & Demonstrated how to achieve high diversity gains in MIMO OFDM systems with the aid of fractional sampling. \\
\hline & Baek et al. [174] & $\begin{array}{l}\text { Addressed a time-domain semi-blind channel estimation approach and a PAPR Reduction scheme for MIMO } \\
\text { OFDM. }\end{array}$ \\
\hline & Dubuc et al. [150] & Outlined Nortel Networks' MIMO OFDM concept prototype and provided measured performance results. \\
\hline & Barriac and Madhow [175] & $\begin{array}{l}\text { Offered guidelines for optimizing the antenna spacing in MIMO OFDM systems using feedback of the covariance } \\
\text { matrix of the downlink channel. }\end{array}$ \\
\hline
\end{tabular}


Table 7 Main Contributions on MIMO OFDM (Part 3)

\begin{tabular}{|c|c|c|}
\hline Year & Author(s) & Contribution \\
\hline \multirow{28}{*}{2005} & $\begin{array}{l}\text { Su, Safar and Liu [137], } \\
\text { [138] }\end{array}$ & $\begin{array}{l}\text { Designed a general space-frequency block code structure capable of providing full-rate, full-diversity MIMO } \\
\text { OFDM transmission. }\end{array}$ \\
\hline & $\begin{array}{l}\text { Zhang, Kavcic and Wong } \\
\text { [176] }\end{array}$ & $\begin{array}{l}\text { Researched an optimal QR decomposition technique designed for a precoded MIMO OFDM system using } \\
\text { successive-cancellation detection. }\end{array}$ \\
\hline & Yao and Giannakis [177] & Proposed a low-complexity blind Carrier Frequency Offset (CFO) estimator for OFDM systems. \\
\hline & Zheng et al. [178] & $\begin{array}{l}\text { Extended Time-Division Synchronous CDMA (TD-SCDMA) to Time-Division Code-Division Multiplexing } \\
\text { OFDM (TD-CDM-OFDM) for future } 4 \mathrm{G} \text { systems. }\end{array}$ \\
\hline & Yang [179] & Reviewed the state-of-the-art approaches in MIMO OFDM air-interface. \\
\hline & Zhang and Letaief [180] & $\begin{array}{l}\text { Aimed at developing an adaptive resource-allocation approach which jointly allocates subcarriers, power and bits } \\
\text { for multi-user MIMO OFDM systems. }\end{array}$ \\
\hline & $\mathrm{Ma}[181]$ & $\begin{array}{l}\text { Established a pilot-assisted modulation scheme for CFO and channel estimation in OFDM transmissions over } \\
\text { frequency-selective MIMO fading channels. }\end{array}$ \\
\hline & $\begin{array}{l}\text { Fozunbal, McLaughlin } \\
\text { and Schafer [182] }\end{array}$ & $\begin{array}{l}\text { Calculated a sphere packing lower bound and a pairwise error upper bound of the error probability of space-time- } \\
\text { frequency coded OFDM systems using multiple antennas for transmission over block-fading channels. }\end{array}$ \\
\hline & Nanda et al. [183] & Built a MIMO WLAN prototype that provides data rates over $200 \mathrm{Mb} / \mathrm{s}$. \\
\hline & Kim et al. [184] & $\begin{array}{l}\text { Invoked a QR-Decomposition combined with the M-algorithm (QRD-M) for joint data detection and channel } \\
\text { estimation in MIMO OFDM. }\end{array}$ \\
\hline & Qiao et al. [185] & Contrived an iterative LS channel estimation algorithm for MIMO OFDM. \\
\hline & $\begin{array}{l}\text { Sampath, Erceg and } \\
\text { Paulraj [186] }\end{array}$ & $\begin{array}{l}\text { Validated the properties of the transmit correlation matrix through field trial results obtained from a MIMO OFDM } \\
\text { wireless system operated in a macro-cellular environment. }\end{array}$ \\
\hline & $\begin{array}{l}\text { Rey, Lamarca and Vazquez } \\
\text { [187] }\end{array}$ & $\begin{array}{l}\text { Used a Bayesian approach to design transmit prefiltering matrices for closed-loop schemes, which is robust to } \\
\text { channel estimation errors. }\end{array}$ \\
\hline & $\begin{array}{l}\text { Sun, Xiong and Wang } \\
{[188]}\end{array}$ & $\begin{array}{l}\text { Targeted at designing CFO estimator aided Expectation Maximization (EM) based iterative receivers for MIMO } \\
\text { OFDM systems. }\end{array}$ \\
\hline & Han and Lee [141] & Provided an overview of multicarrier PAPR reduction techniques. \\
\hline & Lodhi et al. [189] & $\begin{array}{l}\text { Evaluated the complexity and performance of a MC-CDMA system exploiting STBCs and Cyclic Delay Diversity } \\
\text { (CDD). }\end{array}$ \\
\hline & Wang, Han and Liu [190] & Advanced MIMO OFDM channel estimation using a scheme based on estimating the Time-of-Arrivals (TOAs). \\
\hline & $\begin{array}{l}\text { Wen, Wang and Chen } \\
\text { [191] }\end{array}$ & $\begin{array}{l}\text { Reported on a low-complexity multi-user angle-frequency coding scheme based on the Fourier basis structure for } \\
\text { downlink wireless systems. }\end{array}$ \\
\hline & Su, Safar and Liu [192] & Performance analysis of MIMO OFDM systems invoking coding in spatial, temporal and frequency domains. \\
\hline & $\begin{array}{l}\text { Tan, Latinović and Bar- } \\
\text { Ness [193] }\end{array}$ & $\begin{array}{l}\text { Advocated a scheme of cross-antenna rotation and inversion utilizing additional degrees of freedom by employing } \\
\text { multiple antennas in OFDM systems. }\end{array}$ \\
\hline & Park and Cho [194] & $\begin{array}{l}\text { Characterized a MIMO OFDM technique based on the weighting factor optimization for reducing the ICI caused } \\
\text { by time-varying channels. }\end{array}$ \\
\hline & Shao and Roy [195] & $\begin{array}{l}\text { Maximized the diversity gain achieved over frequency-selective channels by employing a full-rate space-frequency } \\
\text { block code for MIMO OFDM systems. }\end{array}$ \\
\hline & Schenk et al. [196] & Quantified how the transmitter/receiver phase noise affects the performance of a MIMO OFDM system. \\
\hline & $\begin{array}{l}\text { Borgmann and } \\
\text { Bölcskei [197] }\end{array}$ & Contributed to the code designs for noncoherent frequency-selective MIMO OFDM fading links. \\
\hline & Tarighat and Sayed [198] & $\begin{array}{l}\text { Examined the effect of IQ imbalances on MIMO OFDM systems and developed a digital signal processing } \\
\text { framework for combating these distortions. }\end{array}$ \\
\hline & Jiang, Li and Hager [199] & $\begin{array}{l}\text { Formulated a joint transceiver design combining the Geometric Mean Decomposition (GMD) with ZF-type } \\
\text { decoders. }\end{array}$ \\
\hline & Choi and Heath [200] & $\begin{array}{l}\text { Constructed a limited feedback architecture that combines beamforming vector quantization and smart vector } \\
\text { interpolation. }\end{array}$ \\
\hline & Baek et al. [201] & Incorporated multiple antennas into high-rate DAB systems. \\
\hline 2006 & Liew and Hanzo [14] & Investigated various space-time codes for OFDM systems. \\
\hline
\end{tabular}

links becomes singular and, hence, noninvertible, thus rendering the degree of freedom of the detector insufficiently high for detecting the signals of all the transmitters in its vicinity. This will catastrophically degrade the performance of numerous known detection approaches, such as for example the Vertical Bell Labs Layered SpaceTime architecture (V-BLAST) [10], [120], [278] detector of [146], the LS/MMSE algorithms of [1], [268] and the QR Decomposition combined with the M-algorithm (QRD-M) algorithm of [184]. However, with the aid of the recently proposed GA-aided MUDs [279]-[284], this problem can be efficiently solved. We will further discuss this issue in Section IV.

\section{B. Channel Estimation}

In MIMO OFDM systems accurate channel estimation is required at the receiver for the sake of invoking both coherent demodulation and interference cancellation. Compared to SISO systems, channel estimation in the MIMO scenario becomes more challenging, since a significantly increased number of independent transmitterreceiver channel links have to be estimated simultaneously for each subcarrier. Moreover, the interfering signals of the other transmitter antennas have to be suppressed.

In the literature, a number of blind channel estimation techniques have been proposed for MIMO OFDM systems [103], [227], [238], [261], [285], where an attempt is made 


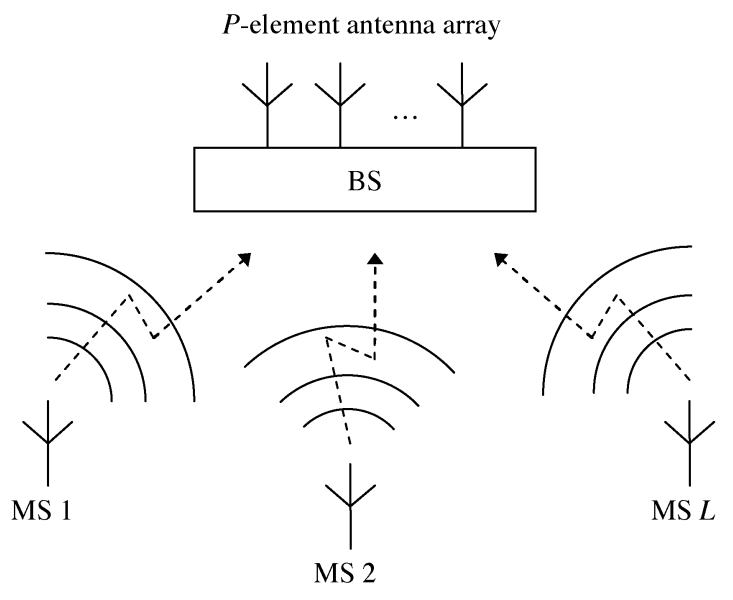

Fig. 6. Illustration of the generic SDMA system employing a $P$-element receiver antenna array for supporting $L$ number of mobile users.

to avoid the reduction of the effective throughout by dispensing with the transmission of known FD channelsounding pilots. However, most of these approaches suffer from either a slow convergence rate or a performance degradation, owing to the inherent limitations of blind search mechanisms. By contrast, the techniques benefiting from explicit training with the aid of known reference/pilot signals, are typically capable of achieving a better performance at the cost of a reduced effective system throughput. For example, Li et al. [286] proposed an approach of exploiting both transmitter diversity and the delay profile characteristics of typical mobile channels, which was further simplified and enhanced in [142], [145], and [287], respectively. Other schemes employed MMSE [136], [288], constrained least-squares (CLS) [209], iterative LS [143], [185], QRD-M [184], [289] as well as second-order statistics (SOS)-based subspace estimation [168] or techniques based on the received signal's time-of-arrival (TOA) [190], etc. Some researchers have focused their attention on designing optimum training patterns or structures [142], [144], [290]. Furthermore, various joint approaches combining channel estimation with data symbol detection at the receiver were also proposed for CDMA [289], [291], SISO OFDM [292] and MIMO OFDM [184], [293] systems. However, in the context of BLAST or SDMA type multiuser MIMO OFDM systems, all channel estimation techniques found in the literature were developed under the assumption of either the underloaded [143], [168], [227], [238], [285], [288], [294] or the fully loaded [144], [184], [190], [209], [261], [293], [295] scenario mentioned above. Unsurprisingly, in rank-deficient MIMO OFDM systems the task of channel estimation becomes extremely challenging, since the associated significant degradation of the rank-deficient MUD's performance will inevitably result in a further degraded performance of the associated channel estimators, especially in decisiondirected type receivers, which are quite sensitive to error propagation [1].

\section{GENETIC ALGORITHM ASSISTED MULTIUSER DETECTION FOR MIMO OFDM}

In rank-deficient multiuser MIMO OFDM systems, the channel's output phasor constellation often becomes linearly nonseparable, rendering multiuser detection a challenging multidimensional optimization problem. As expected, the higher the number of users to be supported, the more challenging the optimization task becomes, simply due to the exponentially increased number of dimensions to be estimated. While most classic techniques [1] suffer from specific limitations in rank-deficient scenarios, we will demonstrate that the family of GAs, which originates from the evolutionary computing research community, can be efficiently incorporated into MIMO OFDM systems and, hence, constitutes a promising solution to the challenging problems discussed in Section III.

\section{A. MIMO SDMA-OFDM Model}

Fig. 8 shows an uplink MIMO SDMA-OFDM system model, where each of the $L$ simultaneous mobile users employs a single transmit antenna, while the BS's receiver exploits $P$ antennas. At the kth subcarrier of the $n$th OFDM symbol received by the $P$-element receiver antenna array, we have the received complex signal vector $\mathbf{x}[n, k]$, which is constituted by the superposition of the independently faded signals associated with the $L$ mobile users and contaminated by the AWGN, expressed as

$$
\mathbf{x}=\mathbf{H s}+\mathbf{n}
$$

where the $(P \times 1)$-dimensional vector $\mathbf{x}$, the $(L \times 1)$ dimensional vector $\mathbf{s}$ and the $(P \times 1)$-dimensional vector $\mathbf{n}$ are the received, transmitted and noise signals, respectively. Here we have omitted the indices $[n, k]$ for each vector for the sake of notational convenience. Specifically, the vectors $\mathbf{X}, \mathbf{s}$, and $\mathbf{n}$ are given by

$$
\begin{aligned}
\mathbf{x} & =\left[x_{1}, x_{2}, \cdots, x_{P}\right]^{T} \\
\mathbf{s} & =\left[s^{(1)}, s^{(2)}, \cdots, s^{(L)}\right]^{T} \\
\mathbf{n} & =\left[n_{1}, n_{2}, \cdots, n_{P}\right]^{T}
\end{aligned}
$$

where $(\cdot)^{T}$ denotes transpose. The $(P \times L)$-dimensional matrix $\mathbf{H}$, which contains the frequency-domain channel 
Table 8 Main Contributions on SDMA (Part 1)

\begin{tabular}{|c|c|c|}
\hline Year & Author(s) & Contribution \\
\hline 1982 & Yeh and Reudink [214] & $\begin{array}{l}\text { Illustrated that high spectrum efficiencies can be achieved in mobile radio systems using a modest number of } \\
\text { space diversity branches. }\end{array}$ \\
\hline 1983 & Ko and Davis [215] & Early studies on SDMA in the context of satellite communication networks. \\
\hline 1989 & $\begin{array}{l}\text { Swales, Beach and Ed- } \\
\text { wards [216], [217] }\end{array}$ & Devised a multi-beam adaptive BS antenna in an attempt to mitigate the problem of limited radio resources. \\
\hline 1990 & $\begin{array}{l}\text { Agee, Schell and Gardner } \\
{[218]}\end{array}$ & Invoked narrowband antenna arrays for blind adaptive signal extraction. \\
\hline 1991 & Anderson et al. [219] & Adopted adaptive antenna techniques to increase the channel capacity. \\
\hline 1992 & $\begin{array}{l}\text { Balaban and Salz [220], } \\
{[221]}\end{array}$ & $\begin{array}{l}\text { Provided a comprehensive characterization of space diversity reception combined with various equalization } \\
\text { techniques. }\end{array}$ \\
\hline \multirow[b]{2}{*}{1994} & $\mathrm{Xu}$ et al. [222] & Offered preliminary results of experimental studies on SDMA systems. \\
\hline & $\begin{array}{l}\text { Talwar, Viberg and } \\
\text { Paulraj [223] }\end{array}$ & Described an approach for separating and estimating multiple co-channel signals with the aid of an antenna array. \\
\hline \multirow{3}{*}{1995} & Van Der Veen et al. [224] & $\begin{array}{l}\text { Blindly identified Finite Impulse Response (FIR) channels using oversampling and the finite-alphabet property of } \\
\text { digital signals. }\end{array}$ \\
\hline & $\begin{array}{l}\text { Khalaj, Paulraj and Kailath } \\
{[225]}\end{array}$ & $\begin{array}{l}\text { Estimated the spatio-temporal characteristics of the radio channel in coherent direct-sequence spread-spectrum } \\
\text { systems. }\end{array}$ \\
\hline & $\begin{array}{l}\text { Anand, Mathew and Reddy } \\
{[226]}\end{array}$ & $\begin{array}{l}\text { Established a method of blind separation of co-channel Binary Phase-Shift Keying (BPSK) signals arriving at an } \\
\text { antenna array. }\end{array}$ \\
\hline \multirow[b]{2}{*}{1997} & Liu and $\mathrm{Xu}[227]$ & Addressed the SDMA uplink blind channel and sequence estimation problem. \\
\hline & $\begin{array}{l}\text { Tsoulos, Beach and } \\
\text { McGeehan [228] }\end{array}$ & $\begin{array}{l}\text { Reported the research of the TSUNAMI project that demonstrated the benefits of SDMA in wireless communi- } \\
\text { cations. }\end{array}$ \\
\hline \multirow{10}{*}{1998} & Deneire and Slock [229] & $\begin{array}{l}\text { Derived a subspace fitting and linear prediction method using cyclic statistics of fractionally sampled channels } \\
\text { for channel identification in multi-user and multi-antenna systems. }\end{array}$ \\
\hline & $\begin{array}{l}\text { Tsoulos, McGeehan and } \\
\text { Beach [230], [231] }\end{array}$ & Provided an experimental demonstration of both transmit and receive beamforming supporting SDMA user access. \\
\hline & Barroso et al. $[232]$ & $\begin{array}{l}\text { Introduced a blind algorithm referred to as Array Channel Division Multiple Access (AChDMA) for advanced } \\
\text { SDMA in mobile communications systems. }\end{array}$ \\
\hline & $\begin{array}{l}\text { Demmerle and Wiesbeck } \\
{[233]}\end{array}$ & Designed a biconical multi-beam antenna structure for SDMA communications. \\
\hline & Lindmark [234] & Built a dual-polarized antenna array for a SDMA system working in the $1850-1990 \mathrm{MHz}$ band. \\
\hline & Suard et al. [235] & Investigated the channel capacity enhancement of a SDMA system. \\
\hline & Jeng et al. [236] & Presented extensive experimental results of spatial signature variation using a smart antenna testbed. \\
\hline & $\begin{array}{l}\text { Petrus, Ertel and Reed } \\
\text { [237] }\end{array}$ & $\begin{array}{l}\text { Proved that capacity improvement can be achieved using adaptive arrays at the BS of an Advanced Mobile Phone } \\
\text { Service (AMPS) system. }\end{array}$ \\
\hline & $\begin{array}{l}\text { Xavier, Barroso and } \\
\text { Moura }[238]\end{array}$ & Targeted at designing a closed-form estimator for the SDMA MIMO channel based on second-order statistics. \\
\hline & Farsakh and Nossek [239] & $\begin{array}{l}\text { Developed an approach for jointly calculating array weights in such a way that all users receive their signal at a } \\
\text { given SINR level. }\end{array}$ \\
\hline \multirow{6}{*}{1999} & Tsoulos [240] & Provided an overview of smart antennas in the context of current and future personal communication systems. \\
\hline & Piolini and Rolando [241] & Analyzed a channel-assignment algorithm for SDMA mobile systems. \\
\hline & $\begin{array}{l}\text { Vandenameele et al. } \\
\text { [202], [206], [207] }\end{array}$ & Advocated a combined SDMA-OFDM approach that couples the capabilities of the two techniques. \\
\hline & $\begin{array}{l}\text { Galvan-Tejada and Gar- } \\
\text { diner [242], [243] }\end{array}$ & $\begin{array}{l}\text { Calculated the theoretical blocking probability resulting from SDMA technology in two different channel allocation } \\
\text { schemes. }\end{array}$ \\
\hline & Tsoulos [244] & Focused on TDMA air interface techniques combined with SDMA schemes. \\
\hline & $\begin{array}{l}\text { Vornefeld, C. Walke and } \\
\text { B. Walke [245] }\end{array}$ & Applied SDMA techniques to WATM systems. \\
\hline
\end{tabular}

transfer functions (FD-CHTFs) of the $L$ users, is given by

$$
\mathbf{H}=\left[\mathbf{H}^{(1)}, \mathbf{H}^{(2)}, \cdots, \mathbf{H}^{(L)}\right]
$$

where $\mathbf{H}^{(l)}(l=1, \cdots, L)$ is the vector of the FD-CHTFs associated with the transmission paths from the lth user's transmit antenna to each element of the P-element receiver antenna array, which is expressed

$$
\mathbf{H}^{(l)}=\left[H_{1}^{(l)}, H_{2}^{(l)}, \cdots, H_{P}^{(l)}\right]^{T}, \quad l=1, \cdots, L .
$$

In (1)-(6), we assume that the complex signal $s^{(l)}$ transmitted by the lth user has zero-mean and a variance of $\sigma_{l}^{2}$. The AWGN signal $n_{p}$ also exhibits a zero-mean and a variance of $\sigma_{n}^{2}$. The FD-CHTFs $H_{p}^{(l)}$ of the different receivers or users are independent, stationary, complex Gaussian distributed processes with zero-mean and unit variance [1].

\section{B. Classic SDMA-OFDM MUDs and Problem Formulation}

1) MMSE MUD [1], [268]: Naturally, the problem formulation and the corresponding objective or cost-function 
as well as the optimization procedure used has a crucial impact on the attainable performance as well as complexity of the MUD. Initially we will briefly outline the computationally simple MMSE SDMA-OFDM MUD, followed by a number of other classic MUDs. As seen in Fig. 9, the multiuser signals received by the different array elements are multiplied by an individual complex-valued array-weight $w^{(l)}$, followed by their summation. The plausible criterion used for array weight adjustment is that of minimizing the difference $\epsilon(t)$ between the combiner's output signal $y(t)$ and a reference signal $r(t)$, which is known to both the transmitter and to the receiver. This signal may be interpreted as a unique userspecific channel-sounding pilot signal employed for example by classic channel equalizers for estimating the CIR. For example the classic steepest descent algorithm my be used for adjusting the array weights on a step-by- step basis for each subcarrier of each user, until the power of the error signal is minimized [1]. Naturally, each user's signal is detected with the aid of a different set of array weights. As expected, the combiner's performance improves upon increasing the number of array elements $P$ and it degrades, when increasing number of users $L$.

2) SIC MUD [1], [268]: In SIC-aided SDMA-OFDM MUDs the following operations are carried out for all subcarriers [1].

a) Arrange the detection order of users upon invoking an estimate of the total signal power received by each individual users on the different antenna elements and detect the highest-power user's signal using the above-mentioned MMSE detector, since it is the least contaminated by multiuser interference (MUI).

Table 9 Main Contributions on SDMA (Part 2)

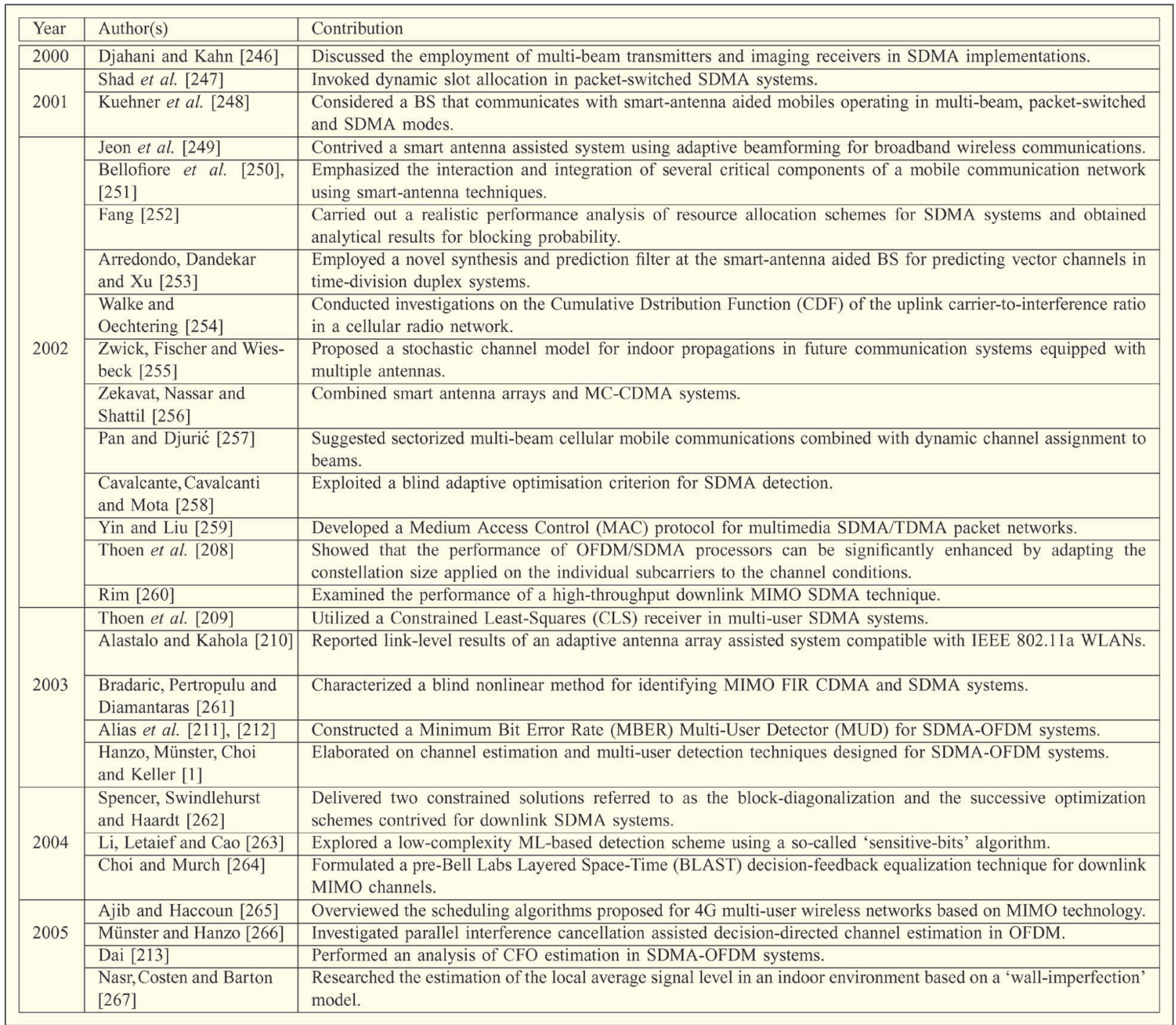




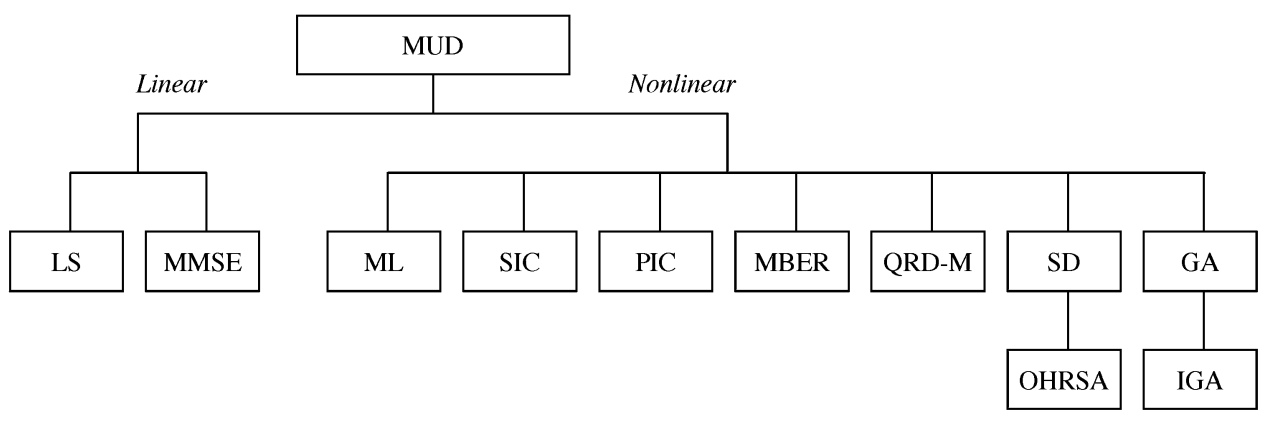

Fig. 7. Various MUDs.

b) Then re-generate the modulated signal of this user from his/her detected date and subtract it from the composite multiuser signal.

c) Detect the next user according to the above procedure by means of MMSE combining, until the lowest-power signal is also detected,

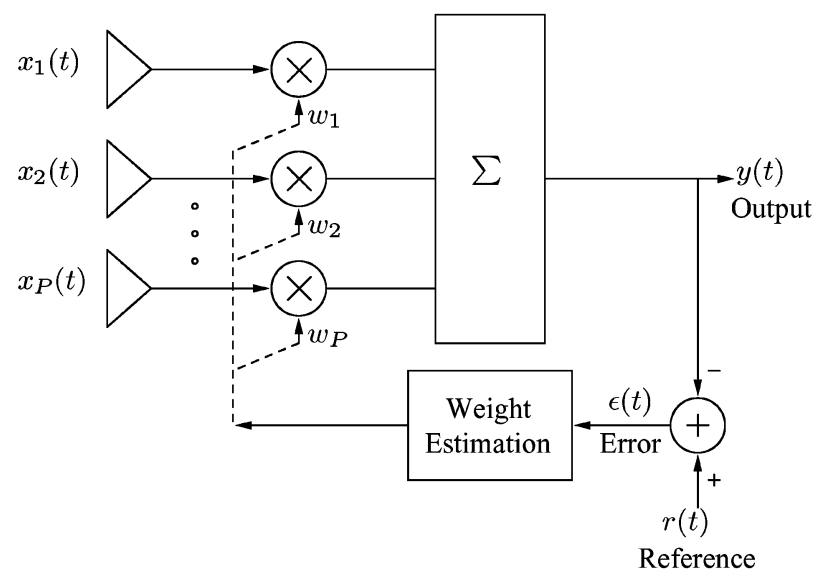

Fig. 9. MMSE SDMA-OFDM MUD schematic. which has by now been "decontaminated" from the MUI.

d) Since the lowest-power user's signal is detected last, a high diversity gain is achieved by the MMSE combiner, which, hence, mitigates not only the the effects of MUI but also that of channel fades.

e) It has to be note, however that the SIC MUD is potentially prone to user-power classification errors as well as to interuser error propagation, since in case of detection errors the wrong remodulated signal is deducted from the composite multiuser signal. Hence, this technique is beneficial in near-far scenarios encountered in the absence of accurate power control.

3) PIC MUD [1], [268]: By contrast, PIC dispenses with user-power classification all together, hence avoiding the above-mentioned potential mis-classification problem. It has benefits in more accurately powercontrolled scenarios. Its philosophy is briefly summarized as follows [1].

a) First detection iteration for all subcarriers:

- on the basis of the composite received multiuser signal vector $\mathbf{x}$ generate an 


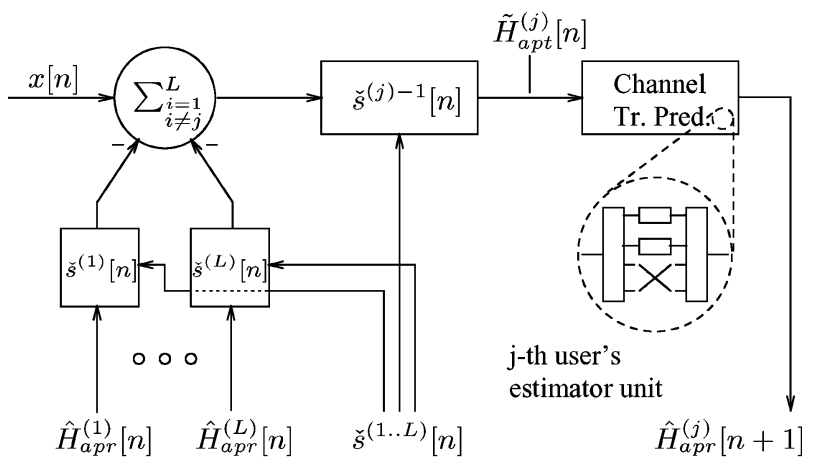

Fig. 10. Illustration of the PIC-assisted channel transfer function estimation -or prediction block - associated with the $j$ th user and any of the $P$ receiver antenna elements [1].

estimate of all user signals $y^{(l)}$ emplying the above-mentioned MMSE detection;

b) if channel encoding was used, decode, slice, and channel encode again as well as remodulate the each user's signal on an OFDM subcarrier basis.

c) Second detection iteration for all subcarriers:

- reconstruct the signal vectors $\check{x}^{(l)}, l \in$ $\{1, \ldots, L\}$ of all users;

- generate an estimate $\tilde{y}^{(k)}$ of all user signals by subtracting the signal vectors $\check{x}^{(l)}, l \neq k$ of all the other users followed by the abovementioned MMSE combining.

d) Channel decode and slice the extracted user signals in order to generate $b^{(l)}$.

e) Despite the absence of power-classification errors the PIC technique is also sensitive to interuser error propagation.

The above-mentioned PIC MUD steps are also summarized in the schematic of Fig. 10, where a FD-CHTF predictor is shown at the top right hand corner, which improves the achievable performance, as it will be detailed below. The channel estimation research of the community was summarized in Section III-B and in the light of these state-of-the-art efforts here a powerful decision-directed channel estimator (DDCE) will be characterized as an example of joint MUD and DDCE. Observe in Fig. 10 that initially the a priori FD-CHTF estimate generated with the aid of a relatively low FD pilot overhead of about $5 \%$ is employed by all the $L$ users. as exemplified at the top right hand corner of Fig. 10 for user $j$. This a priori FD-CHTF allows us to carry out a tentative detection for all users.

The stylized vertical boxes in the circle of Fig. 10 indicate that a complexity reduction technique may be used by the FD-CHTF prediction process. Specifically, the FD-CHTF is first transformed to the TD with the aid of the inverse FFT (IFFT), in order to predict a reduced number of $K_{0} \leq K$ significant CIR taps, instead of having to predict all the $K$ FD-CHTFs of the subcarriers. The large cross at the right of Fig. 10 indicates that the insignificant CIR taps indeed are obliterated, which allows us to reduce the amount of noise imposed. By contrast, the significant CIR taps are predicted, as portrayed in Fig. 11 and then transformed back to the FD using the FFT. Again, even upon taking into account the complexity of the IFFT and FFT, the DDCE complexity is reduced, when $K$ is high.

Then, all but the $j$ th users' received signals are regenerated by taking into account their specific a priori FD-CHTF estimates and the resultant $L-1$ signals are subtracted from the the received composite signal of the $L$ users, which allows the system to reliably detect the "de-contaminated' signal of user $j$. Then the full power of DDCE is exploited, which allows us to use all the reliably detected $K$ subcarrier signals as pilot signals, since in the absence of transmission errors all the transmitted subcarrier data is known. Hence, a simple division of the received signals by the detected signals delivers the a posteriori FD-CHTFs, which can now be fed into the predictor of Fig. 11 for future prediction.

In Fig. 12 we focused our attention on the SDMAOFDM scenario of four simultaneous users, each equipped with one transmit antenna, while at the BS a four-element antenna array is employed. The channel between each transmit-receive antenna pair-characterized in terms of its sample-spaced CIR-and the OFDM parameters are fixed to those used by the indoor WATM system of [2]. Two of the above-mentioned detection techniques were invoked, namely the MMSE and the SIC MUD techniques. The corresponding BER results - following the

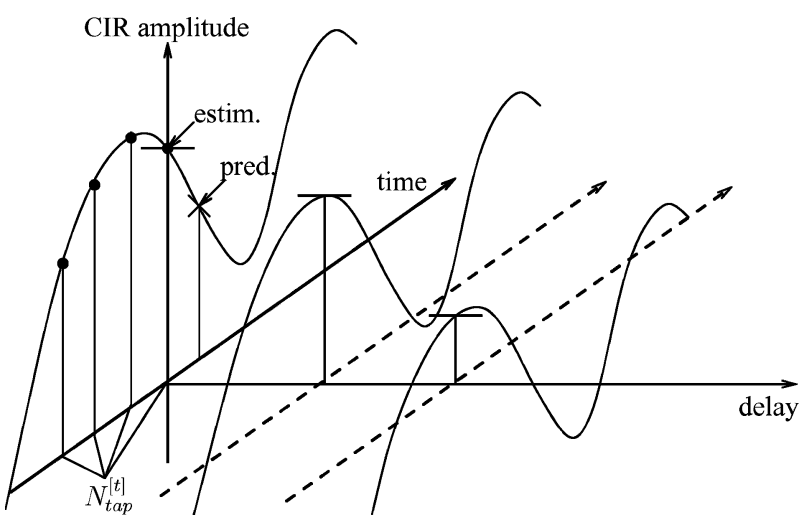

Fig. 11. Stylized illustration of the estimation and prediction filter, both operating in the CIR-related domain using $N_{\text {tap }}^{[t]}$ number of previous a posteriori CIR-related tap estimates. In the context of estimation filtering improved a posteriori tap estimates are generated for the current OFDM symbol period, while in the context prediction filtering a posteriori tap estimates are computed for the next OFDM symbol period [1]. 


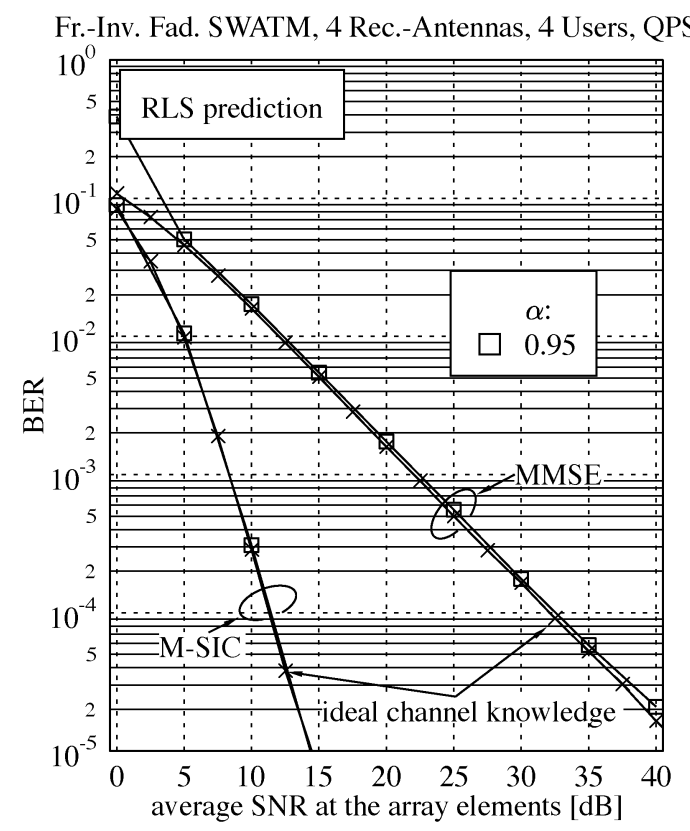

Fig. 12. BER versuS SNR performance associated with the CIR-related tap-by-tap based RLS-adaptive PIC-assisted DDCE of Fig. 10 in the context of a scenario of four receiver antennas at the BS and four simultaneous users, each equipped with one transmit antenna; the channel between each transmitter-receiver antenna pair is characterized in terms of its CIR and the OFDM parameters of the indoor WATM channel- and system scenario of [2] the OFDM symbol normalized Doppler frequency was $F_{D}=0.007$; furthermore, the number of significant CIR-related taps was $K_{0}=12$ and the number of subcarriers was $K=512$; MMSE- as well as M-SIC $(M=2)$ detection was employed at the receiver and both an ideal, error-free reference and an imperfect, error-contaminated reference was invoked in the DDCE; the RLS-specific forgetting factor was set to $\alpha=\alpha_{\mathrm{R} L S}=0.95$.

initial CIR-tap predictor adaptation-are portrayed in Fig. 12 [1].

In the context of the BER performance assessment shown in Fig. 12 we observe that with the aid of the imperfect channel estimates produced by the recursive least square (RLS) adaptive PIC-assisted DDCE almost the same performance is achieved, as in case of employing ideal channel knowledge. This is particularly true for the more powerful SIC MUD, which produces relatively reliable symbol decisions and, hence, also a reliable remodulated reference for the RLS-adaptive PIC-assisted DDCE. By contrast, a slight BER degradation is observed, when using the less powerful MMSE detection scheme, particularly for the range of SNRs up to $5 \mathrm{~dB}$. For higher SNRs the BER performance is also almost identical to that when using perfect channel estimates.

4) $M L$ MUD [1], [268]: The highest-complexity, highestperformance optimum ML MUD [1] uses an exhaustive search for finding the most likely transmitted signals. More explicitly, for a ML-detection assisted SDMA-
OFDM system supporting $L$ simultaneous users, a total of $2^{m L}$ metric evaluations has to be invoked, where $m$ denotes the number of bits per symbol (BPS), in order to detect the $L$-user symbol vector $\hat{\mathbf{s}}_{\mathrm{ML}}$ that consists of the most likely transmitted symbols of the $L$ users at a specific subcarrier, given by

$$
\hat{\mathbf{s}}_{\mathrm{ML}}=\arg \left\{\min _{\check{\mathbf{s}} \in \mathcal{M}^{L}}\|\mathbf{x}-\mathbf{H} \check{\mathbf{s}}\|^{2}\right\}
$$

where the $(P \times 1)$-dimensional received signal vector $\mathbf{x}$ and the $(P \times L)$-dimensional FD-CHTF matrix $\mathbf{H}$ are defined by (2) and (5), respectively. The set $\mathcal{M}^{L}$ in (7), which is constituted by $2^{m L}$ number of trial vectors, is formulated as

$$
\begin{aligned}
\mathcal{M}^{L}=\left\{\check{\mathbf{s}}=\left[\check{s}^{(1)}, \check{s}^{(2)}, \cdots, \check{s}^{(L)}\right]^{T} \mid\right. \\
\left.\check{s}^{(1)}, \check{s}^{(2)}, \cdots, \check{s}^{(L)} \in \mathcal{M}_{c}\right\}
\end{aligned}
$$

where $\mathcal{M}_{c}$ denotes the set containing the $2^{m}$ number of legitimate complex constellation points associated with the specific modulation scheme employed. Explicitly, the number of metric evaluations required for detecting the optimum vector increases exponentially with the number of users $L$.

5) SD-Aided MUD [296]: Another powerful MUD is constituted by the SD [296], which is capable of approaching the performance of the ML MUD at a fraction of its complexity by invoking a focussed search in the vicinity of the likely ML solution. The complexity of the MUD is controlled with the aid of the search-sphere's radius. Damen et al. [297] proposed a powerful SD, which was suitable for rank-deficient MIMO MUDs by detecting the signals received from more transmitters than the number of receive antennas and this schemes was also further developed by by Cui and Tellambura [298]. The complex-valued SD (CSD) was contrived by Hochwald and ten Brink [299] and its was revisited again by Damen et al. in [300] as well as by Pham et al. in [301]. Cui and Tellambura contrived CSD-based joint channel estimation and data detection scheme in [302], while the so-called Multistage Sphere Decoding (MSD) technique was advocated by Cui and Tellambura in [303]. Zhao and Giannakis [304] were capable of reducing the search radius required, which resulted in a reduced complexity.

The radical objective of the rest of this paper is to stimulate further interdisciplinary research for the sake of solving the optimization problem of (7) by 
retaining the optimum ML solution with a high probability, despite searching only a fraction of the entire search space. Finding reduced-complexity nearoptimum MUDs is vital in the light of high-throughput QAM modems employing a high number of antennas. In cases of for example 64 QAM transmissions the number of optimization metric evaluations per subcarrier may become excessive, since it increases exponentially according to $M^{L}$, where $M$ is the number of QAM constellation points. In order to exemplify the optimization problem considered, in Fig. 13 we portray the BER surface of the $L=P=2$ SDMA-OFDM system employing 128subcarriers for transmission over the Short Wireless Asynchronous Transfer Mode (SWATM) CIR given on page 78 of [1] at a SNR value of $10 \mathrm{~dB}$, assuming the optimum value was used for the weight $w$ [56]. Observe that in this scenario the lowest possible BER, which is referred to as the MBER solution has about five orders of magnitude lower BER than the MMSE solution. A powerful direct MBER SDMA-OFDM system was proposed in [212], where the philosophy was that of commencing the search for example from the MMSE solution and estimating the gradient of the BER surface, which was then used for example by the classic Conjugate Gradient Algorithm (CGA) for finding the MBER point on the surface. Naturally, a whole host of random guided search techniques, such as GAs may also be used for exploring a fraction of the search space without getting trapped in local minima, as suggested in [212]. As a last resort, if the search fails to converge, the MMSE solution may be retained throughout the search.

Owing to space limitations we are unable to explore the entire suite of near-optimum search techniques contrived with the above-mentioned objective and, hence, below we will consider the specific family of GA-aided array weight optimization in the interest of finding the MBER point seen in Fig. 13.

\section{Overview of GA-Aided MUDs}

The philosophy of GAs [27]-[31] was first invoked by Holland [27] during the 1960s. Since then, a growing interest in GAs resulted in a rapid development in this area [28], [305], [306]. The random guided search based GAs have been shown to perform well in numerous global search and optimization problems, such as machine learning [307]-[309], adaptive processes modelling [310], [311] as well as many diverse optimization problems [27], [28], [312] which may not be conveniently be solved by using traditional search methods. Currently there are in excess of 12000 diverse GA-related contributions at IEEE Xplore, which indicates the intense interest of numerous research communities in both the theory and applications of GAs. Our objective is here to stimulate the cross-pollination of these diverse communities.

Specifically, in the context of wireless communications, GA-based multiuser detection has been proposed by Juntti et al. [313] and Wang et al. [314], where the analysis was based on the AWGN channel in the absence of diversity techniques. The proposal by Ergün and Hacioglu [315] utilized GAs as the first stage of a multistage MUD, in order to provide good initial guesses for the subsequent stages. Its employment in Rayleigh fading channels was considered by Yen et al. in [25], [316], and [317] in diverse scenarios, both with and without the aid of diversity techniques, respectively. However, apart from

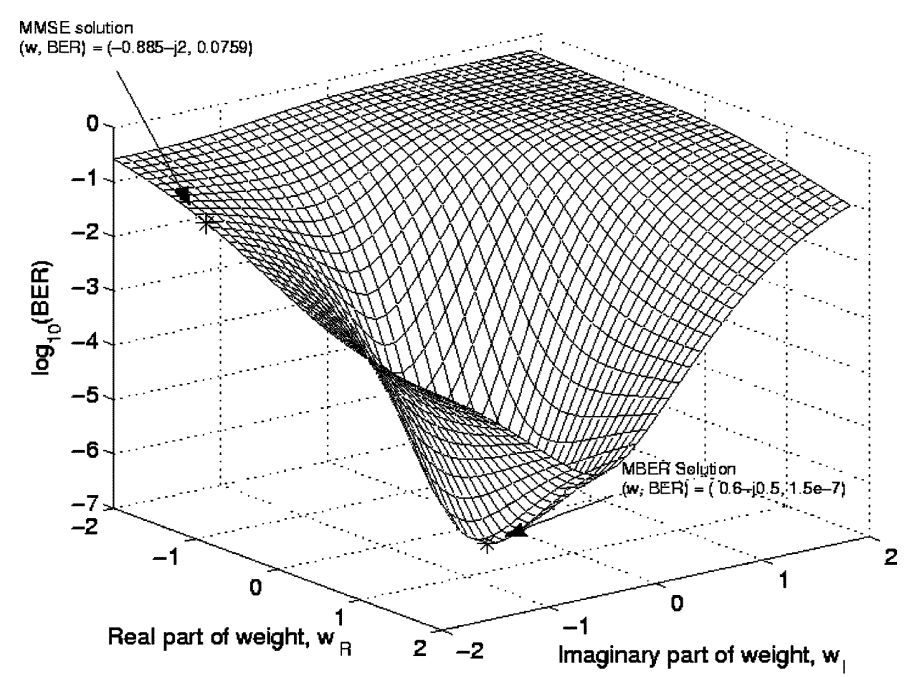

Fig. 13. An example of the BER cost function surface for subcarrier 62 in the $L=P=2$ SDMA-OFDM system employing 128-subcarrier in the SWATM channel at a SNR value of $10 \mathrm{~dB}$ [212]. 
Table 10 Contributions Towards the Development of GA-Aided MuDs

\begin{tabular}{|c|c|c|}
\hline Year & Author(s) & Contribution \\
\hline 1975 & Holland [27] & Originally proposed GAs for studying the adaptive process of natural evolution in an artificial system software. \\
\hline 1989 & Goldberg [28] & Goldberg further developed GAs in the context of optimisation and machine learning. \\
\hline 1996 & Mitchell [29] & A further advancement of GAs in machine learning. \\
\hline 1997 & $\begin{array}{l}\text { Juntti, Schlosser and Lille- } \\
\text { berg [313] }\end{array}$ & First known study of the application of GAs in MUDs. \\
\hline 1998 & $\begin{array}{l}\text { Wang, Lu and Antoniou } \\
{[314]}\end{array}$ & $\begin{array}{l}\text { Proposed a detector for multi-user communications, which is based on the maximum-likelihood decision rule and } \\
\text { employs a GA for detecting the user bits sequentially. }\end{array}$ \\
\hline 2000 & Ergun and Hacioglu [315] & $\begin{array}{l}\text { Suggested a hybrid approach that combines GAs with a multistage multi-user detector (MSD) in the context of } \\
\text { a CDMA system. }\end{array}$ \\
\hline \multirow[b]{2}{*}{2001} & Abedi and Tafazolli [319] & Proposed and characterised a genetic implementation of the optimal MUD. \\
\hline & Yen and Hanzo [317] & $\begin{array}{l}\text { Employed a novel CDMA multi-user receiver based on GAs, for jointly estimating the transmitted symbols and } \\
\text { the fading channel coefficients of all users. }\end{array}$ \\
\hline \multirow[b]{2}{*}{2002} & $\mathrm{Ng}$, Yen and Hanzo [321] & $\begin{array}{l}\text { Advocated a turbo trellis coded modulation assisted GA-aided reduced complexity MUD (TTCM-GA-MUD) that } \\
\text { is capable of providing a considerable coding gain without any bandwidth expansion, while maintaining a low } \\
\text { complexity compared to the optimum MUD. }\end{array}$ \\
\hline & Chiang and Chang [325] & $\begin{array}{l}\text { Improved the GA and MSD using eugenic population. Unlike the elitist selection which ensures that only the } \\
\text { fittest member of the parents' population is retained in the next generation, the eugenic GA combines the offspring } \\
\text { and members of the parents' population into a joint population. Then, the fitness values of all the elements in the } \\
\text { joint population are evaluated and the high-fitness chromosomes are selected to produce the eugenic population. }\end{array}$ \\
\hline \multirow{3}{*}{2003} & Yen and Hanzo [316] & Advocated a spatial diversity reception-assisted CDMA multi-user detector based on GAs. \\
\hline & Du and Chan [322] & Invoked a GA for sub-optimal detection in STBC-aided multi-user detection systems. \\
\hline & $\begin{array}{l}\text { Shayesteh, Menhaj and } \\
\text { Nobary [320] }\end{array}$ & $\begin{array}{l}\text { Proposed a modified genetic algorithm for multi-user detection in DS-CDMA systems, which attains a performance } \\
\text { comparable to that of the optimum detector with at a lower complexity. }\end{array}$ \\
\hline \multirow[b]{2}{*}{2004} & $\begin{array}{l}\text { Wolfgang, Ahmad, Chen } \\
\text { and Hanzo [326] }\end{array}$ & Introduced a novel GA-assisted MBER beamforming technique. \\
\hline & $\begin{array}{l}\text { Alias, Chen and Hanzo } \\
\text { [327] }\end{array}$ & $\begin{array}{l}\text { Employed GAs to determine the MUD's weights for an SDMA OFDM system directly optimizing the MBER } \\
\text { criterion. }\end{array}$ \\
\hline
\end{tabular}

the transceiver research conducted in the context of CDMA systems [25], [318]-[321], GAs have not been exploited to their full potential in wireless communication systems until recently, when they were also applied to STBC-aided MUD scenarios [322], beamforming MIMO detection problems [323] and SDMA-based MIMO OFDM systems [56], [279]-[284], [324]. These promising GA-aided applications are summarized at a glance in Table 10.

The family of GA-based random guided optimization methods is reminiscent of the rules of evolution and to the survival of the fittest "individuals" in nature, where an "individual" in our specific optimization problem is a particular $L$-dimensional MUD weight vector of the $L$ users. Using GA parlance, these individuals representing the MUD weights will then use the genetic operation of "mating" and "mutation" for the sake of developing a new set of GA individuals-MUD weights-for the next generation in the GA. Specifically, the initial "population" represented by the set of individuals would typically contain the MMSE solution, which is routinely retained throughout the entire search for the sake of maintaining an adequate MUD performance even in the low-probability event of poor GA-aided convergence.

\section{GA-Assisted MUDs for MIMO SDMA-OFDM}

The optimum ML-based decision metric of (7) can be used in GA-aided MUDs designed for the sake of detecting the estimated transmitted symbol vector $\hat{\mathbf{s}}_{\mathrm{GA}}$. In the context of the SDMA-OFDM system employing $P$ receiver antenna elements, the decision metric required for the $p$ th receiver antenna, namely the antenna-specific objective function (OF) [25] can be derived from (7), yielding

$$
\Omega_{p}(\check{\mathbf{s}})=\left|x_{p}-\mathbf{H}_{p} \check{\mathbf{s}}\right|^{2}
$$

where $x_{p}$ is the received symbol at the input of the $p$ th receiver at a specific OFDM subcarrier, while $\mathbf{H}_{p}$ is the $p$ th row of the channel transfer function matrix $\mathbf{H}$. Therefore, the decision rule for the optimum MUD associated with the $p$ th antenna is to choose the specific $L$-symbol vector $\check{\mathbf{S}}$, which minimizes the OF given in (9). Thus, the estimated transmitted symbol vector of the $L$ users based on the knowledge of the received signal at the $p$ th receiver antenna and a specific subcarrier is given by

$$
\hat{\mathbf{s}}_{\mathrm{GA}_{p}}=\arg \left\{\min _{\check{\mathbf{s}}}\left[\Omega_{p}(\check{\mathbf{s}})\right]\right\} .
$$

Since there is a total of $P$ number of receiver antennas, the combined OF can be formulated as [279], [280]

$$
\Omega(\check{\mathbf{s}})=\sum_{p=1}^{P} \Omega_{p}(\check{\mathbf{s}})=\|\mathbf{x}-\mathbf{H} \check{\mathbf{s}}\|^{2}
$$

Hence, the decision rule of the GA-aided MUD is to find the specific estimated transmitted $L$-symbol vector $\hat{\mathbf{s}}_{\mathrm{GA}}$ 
that minimizes $\Omega(\mathbf{s})$ in (11) for every OFDM subcarrier considered.

The GAs can be combined with the MMSE MUD for the sake of contriving a more powerful concatenated MMSE-GA MUD [279], [280], which is capable of achieving a similar performance to that attained by the optimum ML MUD at a significantly lower computational complexity, especially at high user loads. The schematic of a concatenated MMSE-GA MUD aided multiuser SDMA-OFDM system is given in Fig. 14. At the transmitter end, as seen at the top of Fig. 14, the forward error correction (FEC) coded signals $s^{(l)}(l=1, \cdots, L)$ of the $L$ users are forwarded to the IFFT-based OFDM modulators, followed by their transmission over the SDMA MIMO channel of Fig. 8 by the userspecific MSs. At the BS, FFT-based OFDM demodulation is invoked at each element of the receiver antenna array shown at the bottom of Fig. 14. The demodulated outputs $x_{p}(p=1, \cdots, P)$ are then forwarded to the concatenated MMSE-GA MUD for separating the different users' signals, followed by the independent FEC decoders.

1) Concatenated MMSE-GA MUD: In the concatenated MMSE-GA MUD [279], [280], the GA's optimization process exploits the symbol estimates generated by the MMSE MUD as initial information. More specifically, the MMSE-based estimated signal vector $\hat{\mathbf{s}}_{\mathrm{MMSE}} \in \mathbb{C}^{L \times 1}$ is obtained by linearly combining the signals received by the $P$ different receiver antenna elements with the aid of the MMSE array weight matrix, as follows [1]

$$
\hat{\mathbf{s}}_{\mathrm{MMSE}}=\mathbf{W}_{\mathrm{MMSE}}^{\mathrm{H}} \mathbf{x}
$$

where $(\cdot)^{H}$ denotes the Hermitian transpose, and $\mathbf{W}_{\text {MMSE }} \in \mathbb{C}^{P \times L}$ is the MMSE-based weight matrix

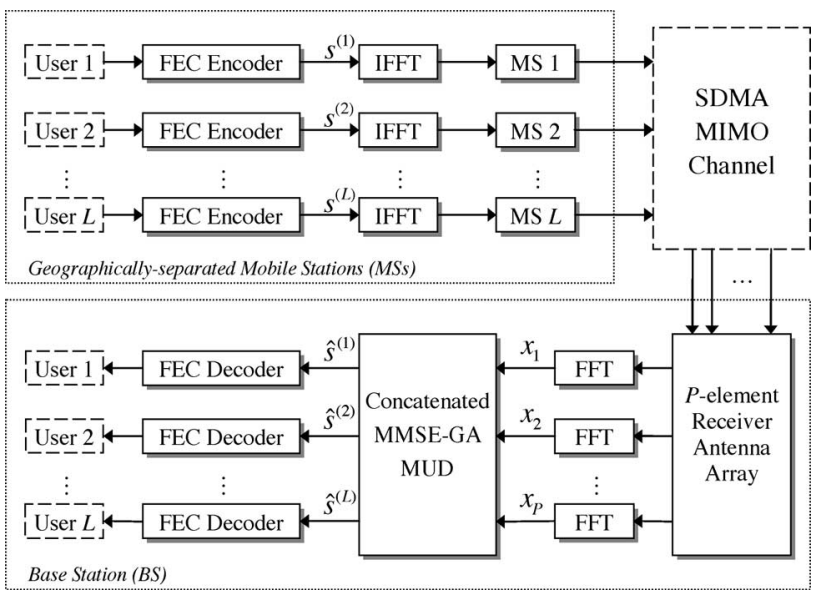

Fig. 14. Schematic of the MMSE-GA-concatenated multiuser detected SDMA-OFDM uplink system. given by [1]

$$
\mathbf{W}_{\mathrm{MMSE}}=\left(\mathbf{H} \mathbf{H}^{H}+\sigma_{n}^{2} \mathbf{I}\right)^{-1} \mathbf{H}
$$

while $\mathbf{I}$ is the identity matrix and $\sigma_{n}^{2}$ is the AWGN noise variance. When the MMSE-estimated $L$-user vector $\hat{\mathbf{s}}_{\text {MMSE }}$ becomes available, the $y=1^{\text {st }}$ GA generation can be created, which contains a population of $X$ individuals. The $x$ th $(x=1, \cdots, X)$ individual is a vector expressed as

$$
\begin{aligned}
\tilde{\mathbf{s}}_{(y, x)} & =\left[\tilde{s}_{(y, x)}^{(1)}, \tilde{s}_{(y, x)}^{(2)}, \cdots, \tilde{s}_{(y, x)}^{(L)}\right], \\
x & =1, \cdots, X ; y=1, \cdots, Y
\end{aligned}
$$

where $Y$ is the maximum generation index. Note that we have the genes $\tilde{s}_{(y, x)}^{(l)} \in \mathcal{M}_{c}(l=1, \cdots, L)$, implying that a complex-valued symbol representation of the individuals is employed. Commencing with the first generation, the GA's search procedure illustrated in Fig. 15 can be invoked.

More explicitly, the GA-based optimization selects some of the $L$-symbol candidates from a total of $X$ legitimate individuals in order to create a so-called mating pool of $T$ number of $L$-symbol individuals [25], as shown in Fig. 15. The selection process follows some specific criterion, for example the strategy of Pareto Optimality [28], [280]. Two individuals in the mating pool are then selected as parents, based on their corresponding fitness values calculated with the aid of (11) according to the socalled fitness-proportionate selection scheme [25]. More precisely, first the fitness value $f_{(y, x)}$ associated with the $x$ th individual of the $y$ th generation is calculated by

$$
f_{(y, x)}=\Omega_{y, T}-\Omega\left(\tilde{\mathbf{s}}_{(y, x)}\right)+c
$$

where

$$
\Omega_{y, T}=\max _{t \in\{1, \cdots, T\}}\left\{\Omega\left(\tilde{\mathbf{s}}_{(y, t)}\right)\right\}
$$

is the maximum objective score $(O S)^{1}$ achieved by evaluating the $T$ number of individuals in the mating pool at

\footnotetext{
${ }^{1}$ Note that the individual having the maximum OS out of the pool of the $T$ candidates is considered as the worst solution in the context of the current mating pool, since the GA searches for the optimum solution which minimizes (11).
} 


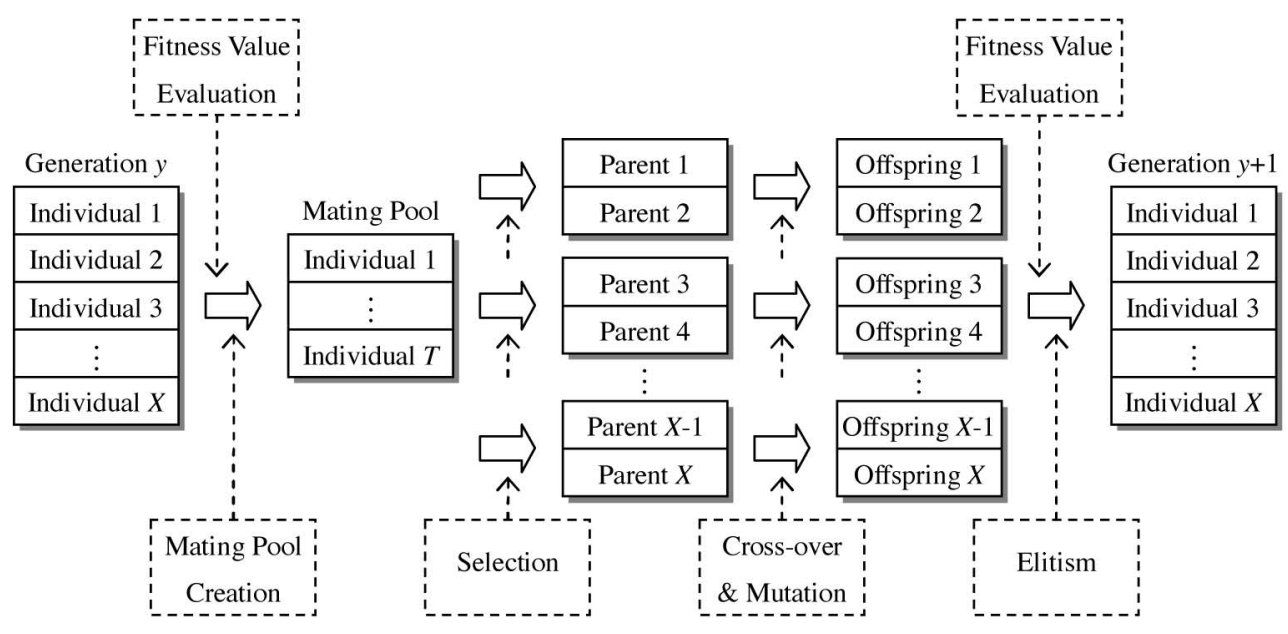

Fig. 15. The GA-based search procedure during one generation.

the $y$ th generation, and $c$ is a small positive constant, which is used for the sake of ensuring the positiveness of $f_{(y, x)}$. Then the fitness-proportionate selection probability $p_{x}$ of the $x$ th individual can be formulated as

$$
p_{x}=\frac{f_{(y, x)}}{\sum_{t=1}^{T} f_{(y, t)}} .
$$

When two L-symbol parents are selected, the so-called cross-over, mutation and elitism operations [25] are invoked for offering a chance of evolving the parents' one or more element symbols to other symbols of the set $\mathcal{M}_{c}$, resulting in two offspring. The above operation is repeated, until a new population consisting of $X$ offspring is created. Furthermore, the so-called incest prevention [25] technique was invoked during the selection process, which only allows different individuals to be selected for the crossover operation. This "genetic evolution-like" process of generating new $L$-symbol offspring continues over $Y$ consecutive GA generations, while the individual having the highest fitness value will be considered as the detected L-user transmitted symbol vector associated with the specific OFDM subcarrier considered. It is worth pointing out that in the MMSE-GA MUD the different users' signals are jointly detected, which is different from that of the SIC or PIC MUDs, where each user's estimated transmitted signal is inferred by removing the interference imposed by the others. Therefore, there is no error propagation between the different users' signal detections in the MMSE-GA MUD.

2) Example: As an example, let us consider a MIMO SDMA-OFDM system using the concatenated MMSE-GA
MUD. The channel is assumed to be "OFDM-symbolinvariant," implying that the taps of the impulse response are assumed to be constant for the duration of one OFDM symbol, but they are faded at the beginning of each OFDM symbol. The simulation results were obtained using a 4 QAM scheme communicating over the threepath SWATM CIR given on page 78 of [1], assuming that the channels' transfer functions are perfectly known. Each of the paths experienced independent Rayleigh fading having the same normalized Doppler frequencies of $f_{d}^{\prime}=1.235 \times 10^{-5}$. The OFDM modem employed $K=512$ subcarriers and a cyclic prefix of 64 samples, which was longer than the maximum channel delay spread. The corresponding parameters of the channel are summarized in Table 11. The FEC scheme was a half-rate iterative Turbo Trellis Coded Modulation (TTCM) scheme [6] using a code memory $\nu=3$, while the number of iterations was set to 4 . Hence, the total number of trellis states was $2^{3} \cdot 4 \cdot 2=64$, since there were two 8-state decoders which were invoked in four iterations. The generator polynomial expressed in octal format for the TTCM scheme considered was [13 6], while the codeword length and channel interleaver depth were fixed to 1024 symbols. The various techniques and parameters used in our simulations discussed in this section are summarized in Table 12.

Table 11 Sampling Rate $1 / T_{s}$, Maximum Path Delay $\tau_{\max }$, Maximum Doppler Frequency $f_{d}$, Normalized Doppler Frequency $f_{d}^{\prime}$, Number of Paths $n$, FFT Length $K$ and Cyclic Prefix Length $c p$ of the SWATM Channel [1]

\begin{tabular}{|c|c|c|c|c|c|c|}
\hline $1 / T_{s}$ & $\tau_{\max }$ & $f_{d}$ & $f_{d}^{\prime}$ & $n$ & $K$ & $c p$ \\
\hline \hline $225 \mathrm{MHz}$ & $48.9 \mathrm{~ns}$ & $2278 \mathrm{~Hz}$ & $1.235 \times 10^{-5}$ & 3 & 512 & 64 \\
\hline
\end{tabular}


Table 12 The Various Techniques and Parameters Used in the Example of Section IV-D2

\begin{tabular}{|c|c|c|}
\hline \multirow{7}{*}{ TTCM } & Modem & 4QAM \\
\hline & Code rate & 0.5 \\
\hline & Code memory $\nu$ & 3 \\
\hline & Octal generator polynomial & {$\left[\begin{array}{ll}13 & 6\end{array}\right]$} \\
\hline & Codeword length & 1024 symbols \\
\hline & Channel interleaver depth & 1024 symbols \\
\hline & Number of turbo iterations & 4 \\
\hline \multirow{10}{*}{ GA } & Population initialization method & MMSE \\
\hline & Mating pool creation strategy & Pareto-Optimality [28] \\
\hline & Selection method & Fitness-Proportionate \\
\hline & Cross-over & Uniform cross-over \\
\hline & Mutation & $M$-ary mutation \\
\hline & Mutation probability $p_{m}$ & 0.1 \\
\hline & Elitism & Enabled \\
\hline & Incest prevention & Enabled \\
\hline & Population size $X$ & Varied \\
\hline & Generations $Y$ & Varied \\
\hline
\end{tabular}

Fig. 16 shows the BER performance of the TTCMassisted MMSE-GA-SDMA-OFDM prototype system, where $L=6$ users are supported with the aid of $P=6$ receiver antenna elements. The performance of the TTCM-assisted MMSE-detected SDMA-OFDM system, the TTCM-aided optimum ML-detected system, and the uncoded single-user scheme employing either a single receiver or invoking maximum ratio combining (MRC) when communicating over an AWGN channel are also provided for reference, respectively. The numbers in the round brackets seen in the legends of Fig. 16 represent the total complexity, which is quantified as the number of evaluations of the OF formulated in (11) required by the MMSE-GA ${ }^{2}$ or ML MUD. Explicitly, it is observed from Fig. 16 that the BER performance of the TTCM-assisted MMSE-SDMA-OFDM system was significantly improved with the aid of the GA having a sufficiently large GA population size $X$ and/or a larger number of GA generations $Y$, approaching the optimum ML-aided performance. This improvement was achieved, since a larger population may contain a higher variety of $L$-symbol individuals, and similarly, a larger number of generations implies that again, a more diverse set of individuals may be evaluated, thus extending the GA's search space, which may be expected to increase the chance of finding a lowerBER solution.

\footnotetext{
${ }^{2}$ The complexity of the simple MMSE MUD has been ignored, since it is used for providing a single initial solution for the GA's initial population and imposes a significantly lower complexity than that of its concatenated GA-aided counterpart.
}

TTCM-MMSE-GA-SDMA-OFDM, L6/P6, 4QAM, SWATM

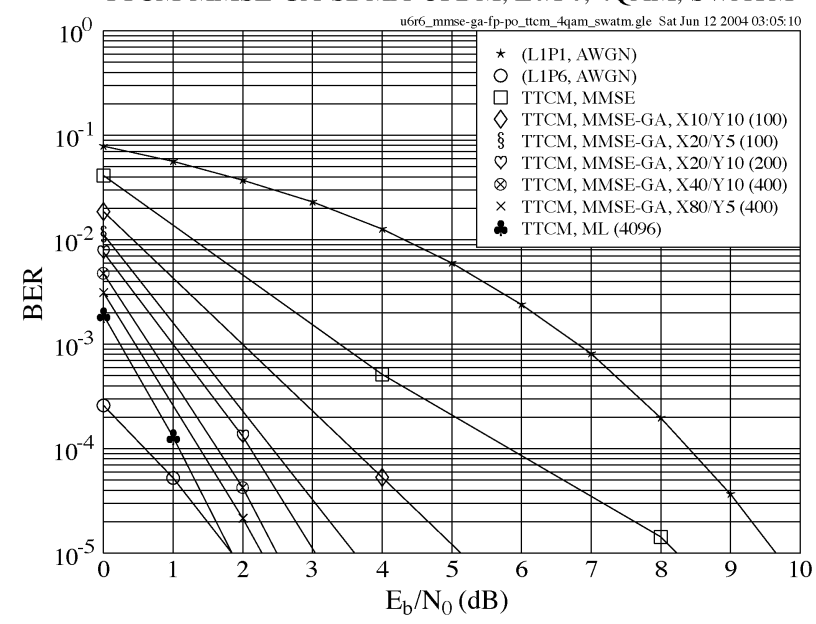

Fig. 16. BER versus $E_{b} / N_{0}$ performance of the TTCM-assisted MMSE-GA-SDMA-OFDM system employing a 4 QAM scheme for transmission over the SWATM channel, where $L=6$ users are supported with the aid of $P=6$ receiver antenna elements. The basic simulation parameters are given in Table 12.

Moreover, it can be seen in Fig. 16 that the nearoptimum performance of the GA-aided TTCM-SDMAOFDM system was achieved at a significantly lower computational complexity of 400 OF evaluations than that imposed by the ML-aided system, which is 4096 OF evaluations. As a further investigation, in Fig. 17 we

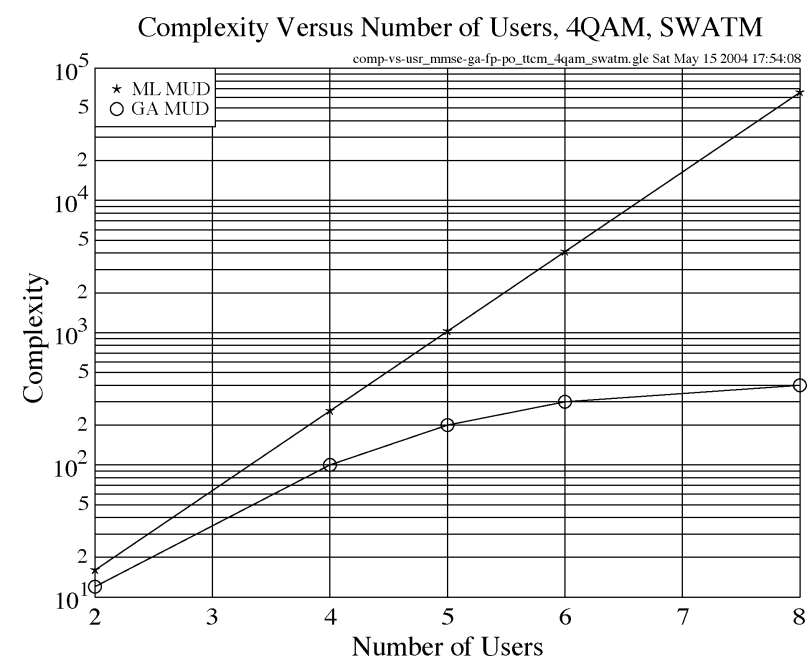

Fig. 17. Comparison of the MUD complexity in terms of the number of OF evaluations, versus the number of users performance of the 4 QAM TTCM-MMSE-GA-SDMA-OFDM and TTCM-ML-SDMA-OFDM systems. The number of receiver antenna elements employed is equivalent to the number of users supported, i.e., $L=P$. 


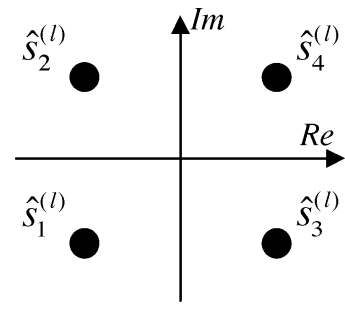

Fig. 18. A 4 QAM constellation.

compare both the ML- and the GA-aided schemes in terms of their complexity, i.e., the number of OF computations. At a specific user load, we always select an appropriate GA-aided scheme for comparison, which suffers from less than $1 \mathrm{~dB} E_{b} / N_{0}$ loss at the BER of $10^{-5}$ compared to the ML-aided system. As shown in Fig. 17, the ML-aided system imposes an exponentially increasing complexity on the order of $O\left(2^{m L}\right)$, when the number of users increases, while the complexity of the GA-aided system required for maintaining a near-optimum performance increases only slowly.

\section{E. Enhanced GA MUDs}

In order to further improve the achievable performance of MIMO SDMA-OFDM systems, the enhanced GA-based MUDs of [281], [282] can be employed, which improve the detector's performance by each or both of the following two aspects.

- Optimizing the GA mutation operator for the sake of finding a better configuration that may improve the GA's performance in the context of the SDMAOFDM system.

- Invoking an iterative detection framework so that the system's performance may be improved iteration by iteration.

1) Improved Mutation Scheme: In the context of GAassisted multiuser SDMA-OFDM systems, when the number of users $L$ increases or a high-throughput modulation scheme is used, the total search space consisting of $2^{m L}$ number of $L$-user symbol vectors would become excessive. In such cases, the role of mutation may become vital for the success of the overall system, since the GA may get trapped in local optima without appropriate assistance of the mutation scheme.

In conventional GAs [25], [279], [280], [316], [317], the so-called uniform mutation (UM) [29] technique is widely used. To elaborate a little further, let us assume a multiuser SDMA-OFDM system employs the 4 QAM signal constellation shown in Fig. 18. Recall furthermore that each of the $L$ genes of a GA individual is represented by a legitimate symbol in $\mathcal{M}_{c}$. During the genetic evolution, when a gene is subjected to mutation, it will be substituted by a different symbol in $\mathcal{M}_{c}$ based on a uniform mutation-induced transition probability $p_{m t}^{(i j)},{ }^{3}$ which quantifies the probability of the ith legitimate symbol becoming the $j$ th. For the sake of brevity, from now on we refer to this probability as the transition probability. Conventionally, the UM operator mutates a gene to another legitimate symbol according to a uniformly distributed $p_{m t}^{(i j)}$ value, which depends on the specific modem employed. For example, for the 4 QAM modem of Fig. 18, we will have ${ }^{4} p_{m t}^{(i j)}=1 /(4-1)=1 / 3$. However, this fixed uniform transition probability fails to reflect the realistic channel conditions that the system is subjected to. More specifically, when considering a specific received symbol, the adjacent constellation symbols are more likely to be the transmitted symbol, than the more distant ones. Hence, it may be more reasonable to consider only the neighboring symbols as the potential mutation candidates, and assign a modified biased transition probability, which is dependent on both the Euclidean distance from the original symbol and on the SNR. In other words, the GA's search space may be substantially reduced with the aid of a biased mutation, which pays less attention to the constellation points that are far from the received symbol, and thus increasing the GA's efficiency.

By contrast, the novel mutation scheme of [281], [282] provides an simple and efficient solution, which is referred to as biased Q-function-based mutation (BQM). According to $\mathrm{BQM}$, for an original gene to be mutated, a SNR-related biased transition probability $p_{m t}^{(i j)}$ will be assigned to each of the target candidate symbols in $\mathcal{M}_{c}$. The calculation of $p_{m t}^{(i j)}$ may be carried out with the aid of the widely known $Q$-function [328]

$$
Q(x)=\frac{1}{\sqrt{2 \pi}} \int_{x}^{\infty} e^{-t^{2} / 2} d t, \quad x \geq 0 .
$$

For the sake of easy explanation, let us first consider a simple 1-D scenario. In Fig. 19 we plotted the 1-D real component of the constellation symbols $\hat{s}_{i}^{(l)}$ in the context of the 4 QAM modem constellation. The horizontal axis is then divided into two zones, each of which represents one specific 1-D constellation symbol $s_{R i}(i=1, \ldots, 2)$, as separated by the vertical dashed line of Fig. 19. If $s_{R 1}$ is the original gene to be mutated, the

\footnotetext{
${ }^{3}$ Note that the mutation probability $p_{m}$ of Table 12 is different from the probability $p_{m t}^{(i)}$ of mutating to a specific symbol in $\mathcal{M}_{c}$. The former denotes the probability of how likely it is that a gene will mutate, while the latter specifies, how likely it is that a specific symbol in $\mathcal{M}_{c}$ becomes the mutated gene.

${ }^{4}$ Note that a gene is not allowed to mutate to itself.
} 


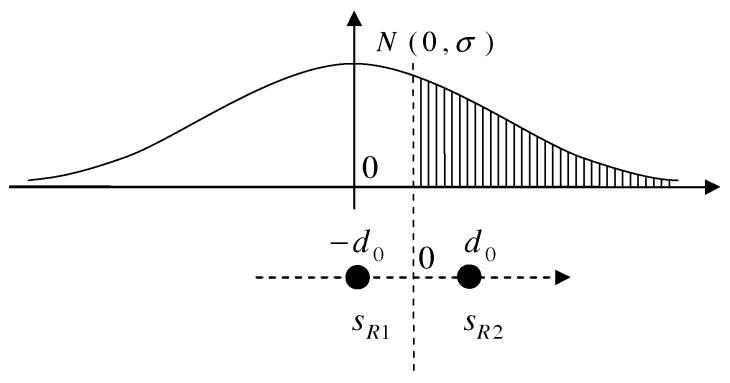

Fig. 19. Illustration of the 1-D transition probability $p_{m t}^{(i j)}$ for 4 QAM.

Gaussian distribution $N(0, \sigma)$ may be centered at the position of $s_{R 1}$, where $\sigma$ is the noise variance at a given $\mathrm{SNR}$ level. In this specific example, $s_{R 2}$ is the only mutation target and the 1-D transition probability of mutating from $s_{R 1}$ to $s_{R 2}$, i.e., $p_{m t}^{(12)}$, is characterized by the shadow area shown in Fig. 19, which is given by

$$
p_{m t}^{(12)}=Q\left(\frac{d_{0}}{\sigma}\right)
$$

where $d_{0}$ is half of the distance between the neighboring constellation symbols. Similarly, we have $p_{m t}^{(\underline{21})}=Q\left(d_{0} / \sigma\right)$. Furthermore, we also have a certain probability for the original gene to remain unchanged, which can also be expressed as $p_{m t}^{(11)}=p_{m t}^{(22)}=1-Q\left(d_{0} / \sigma\right)$. Hence, the corresponding 2-D symbol transition probability $p_{m t}^{(i j)}$ can be derived by combining the 1-D real and imaginary transition probabilities. For the specific 1-D-based 4 QAM example of Fig. 19, we plot the corresponding 2-D constellation in Fig. 20. In Fig. 20, for instance, the 2-D transition probability of mutating from the constellation symbol $\hat{s}_{1}^{(l)}$ to $\hat{s}_{2}^{(l)}$, namely $p_{m t}^{(12)}$, can be calculated by

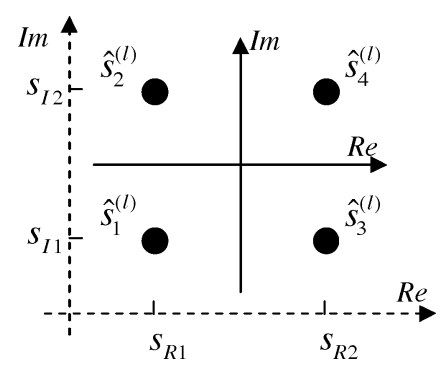

Fig. 20. Illustration of the 2-D transition probability $p_{m t}^{(i j)}$ for 4 QAM, which is the product of the relevant 1-D transition probabilities. $s_{R i}$ and $s_{I i}(i=1,2)$ denote the 1-D constellation symbols in the context of the real and imaginary components of the 4 QAM constellation symbols, respectively. multiplying the two relevant 1-D transition probabilities according to

$$
p_{m t}^{(12)}=p_{m t}^{(11)} \cdot p_{m t}^{(12)}=\left(1-Q\left(\frac{d_{0}}{\sigma}\right)\right) \cdot Q\left(\frac{d_{0}}{\sigma}\right)
$$

while the associated 2-D probability of remaining in the current state is $p_{m t}^{(11)}=p_{m t}^{(11)} \cdot p_{m t}^{(\underline{11})}=\left(1-Q\left(d_{0} / \sigma\right)\right)^{2}$. In order to remove the effect of the probability of mutating a symbol to itself, the 2 -D transition probability $p_{m t}^{(i j)}(i \neq j)$ should be normalized with $p_{m t}^{(i i)}$ by following the principles of conditional probability theory [329].

Note that the BQM scheme can be effectively simplified, when only a subset of all the theoretically possible mutation target symbols are considered [281], [282]. This is especially beneficial for systems employing high-order modems consisting of a large number of legitimate symbols. Furthermore, it is pointed out that the BQM scheme only requires a modest "once-for-all" calculation, since the associated transition probabilities can be derived by offline precomputation for typical SNR levels, which can then be stored in the BS's memory for reuse [281], [282].

2) GA-Aided Hybrid Iterative Detection: The performance of the multiuser SDMA-OFDM system employing the BQM-aided GA MUD can be further improved, when an enhanced iterative detection architecture is utilized [281], [282], which is referred to as the MMSE-initialized Iterative GA (IGA) MUD. The concept of the MMSE-IGA MUD is illustrated in Fig. 21, where TTCM is employed as the embedded FEC decoder. More specifically, the received length- $P$ symbol vector $\mathbf{x}$ of (2) is first detected by the MMSE MUD, which outputs the L MMSE-detected symbols $\hat{s}_{\mathrm{MMSE}}^{(l)}(l=1, \ldots, L)$ of the $L$ users, and forwards them to $L$ number of independent TTCM decoders. The TTCM-decoded $L$-symbol vector, which is more reliable than the MMSE MUD's output, is then fed into the concatenated GA MUD for assisting the creation of the initial population. Then the genetically enhanced output symbol vector $\hat{\mathbf{s}}_{\mathrm{GA}}$, which may be expected to become more reliable, will be fed back to the TTCM decoders in order to further improve the signal's quality, invoking a number of iterations. Following the last iteration, the final GA solution will be decoded by the TTCM decoders, and the hard-decision version of the estimated information bits of the $L$ independent users is forwarded to the output, which is only enabled at the final iteration by the switch seen in Fig. 21.

Therefore, two improvements have been achieved by the MMSE-IGA MUD. Firstly, a more accurate initial knowledge of the transmitted signals, namely the output of 


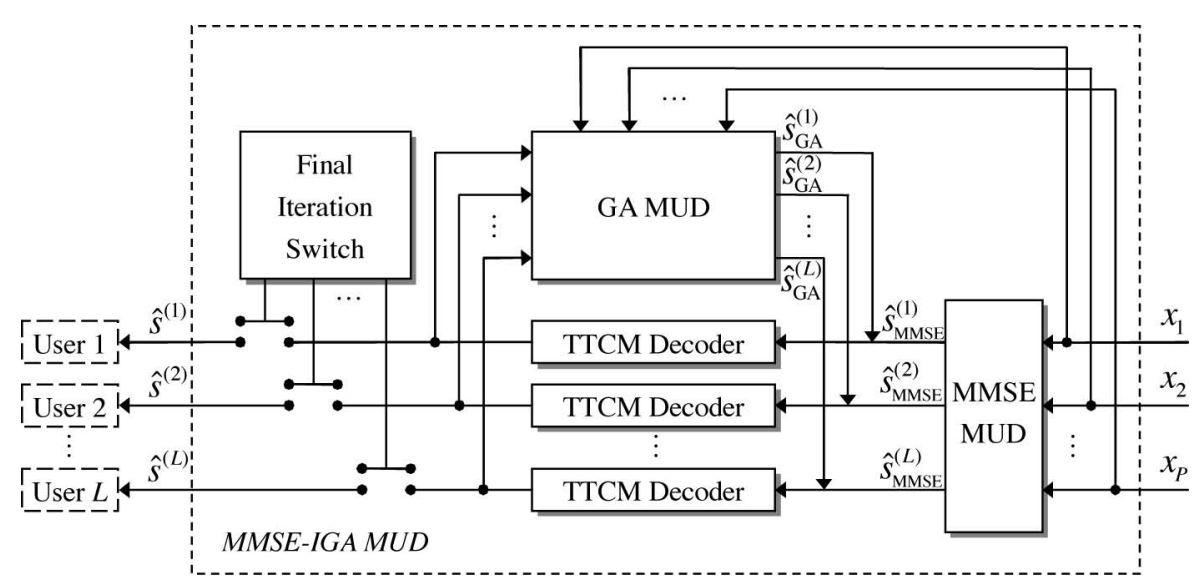

Fig. 21. Structure of an example IGA MUD used at the BS.

the TTCM decoders rather than that of the MMSE MUD, is supplied for the GA MUD. This reliable improvement, therefore, offers a better starting point for the GA's search. Secondly, the iterative processing ensures that the detected $L$-user symbol vector can be optimized in two dimensions. During every iteration, on one hand, each $L$ symbol vector at a specified subcarrier slot is optimized by the GA in the context of the user domain. On the other hand, the entire TTCM-coded frame of each user is optimized by the TTCM decoder in the context of the TTCM-related codeword domain, or more specifically the FD. Therefore, as the iterative processing continues, an information exchange takes place between the two domains and, thus, an improved system performance may be expected.

3) Example: Let us consider our prototype system of Section IV-D2, again, but this time with the employment of the BQM technique and the MMSE-IGA MUD [281], [282]. Note that for the sake of fairness, we halved the number of TTCM decoding iterations for the IGA-aided scheme, so that the total TTCM-related complexity remains approximately the same as in the noniterative system. The other parameters used for generating the simulation results in this section were the same as those specified in Table 12. Fig. 22 shows the BER performance achieved by the various configurations considered. The numbers in the round brackets seen in the legends of Fig. 22 denote the associated number of IGA MUD iterations and the total GA or ML complexity, respectively. It is observed from Fig. 22 that firstly, an improved performance can be achieved, when the GA commences its operation from a better initial population by exploiting the TTCM decoders' outputs, rather than by directly exploiting the MMSE-detected estimates, regardless of the different mutation schemes used. For example, at the same GA complexity of 100 OF evaluations, the single- iteration IGA MUD assisted systems outperformed their noniterative GA aided counterparts. Secondly, the BQMaided systems achieved a better performance than the UM-aided schemes, since BQM is more efficient in guiding the GA towards the optimum solution, as seen in Fig. 22. More explicitly, the system employing the BQMaided two-iteration IGA MUD was capable of achieving a virtually indistinguishable performance from that of the optimum ML-aided system.

When a high-throughput modem such as for example 16 QAM is employed, BQM may significantly outperform UM, as evidenced in Fig. 23, where six users were

TTCM-MMSE-IGA-SDMA-OFDM, L6/P6, 4QAM, SWATM

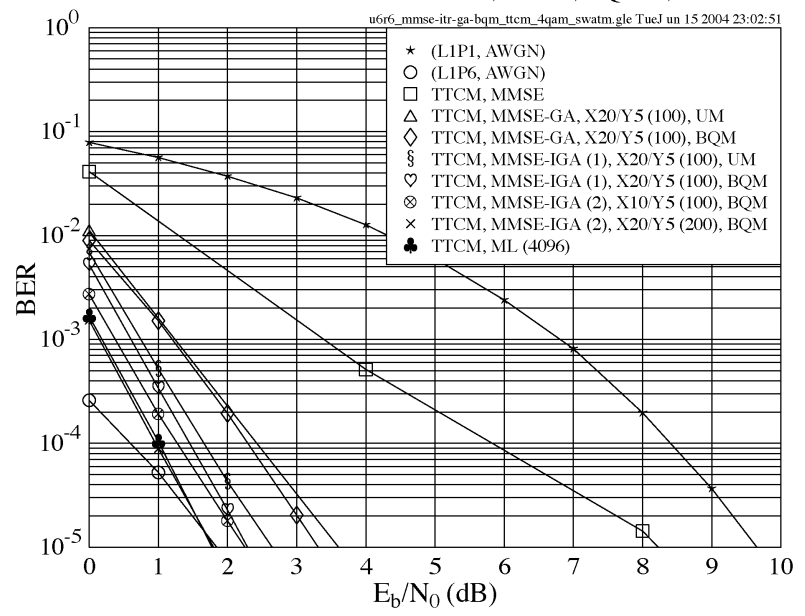

Fig. 22. BER versus $E_{b} / N_{0}$ performance comparison of the iterative or noniterative TTCM-assisted MMSE-GA-SDMA-OFDM system using UM or BQM, while employing a 4 QAM scheme for transmission over the SWATM channel, where $L=6$ users are supported with the aid of $P=6$ receiver antenna elements, respectively. 
TTCM-MMSE-IGA-SDMA-OFDM, L6/P6, 16QAM, SWATM

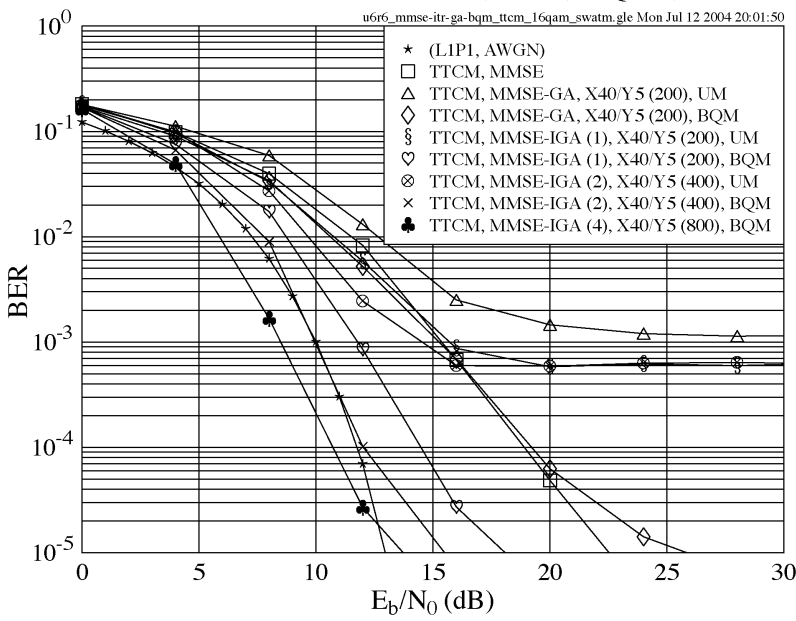

Fig. 23. BER versus $E_{b} / N_{0}$ performance comparison of the iterative or noniterative TTCM-assisted MMSE-GA-SDMA-OFDM system using UM or BQM, while employing a 16 QAM scheme for transmission over the SWATM channel, where $L=6$ users are supported with the aid of $P=6$ receiver antenna elements, respectively.

supported. ${ }^{5}$ As seen in Fig. 23, even when the IGA was employed, the UM-aided scheme yielded a high residual error floor due to the less efficient mutation strategy. By contrast, BQM significantly improved the GA's performance by lowering the error floor by about two orders of magnitude. Furthermore, when the number of IGA MUD iterations was increased, the performance of the BQMaided system can be dramatically improved, while the UMaided scheme still suffered from an error floor. This suggests that the BQM-aided scheme is capable of substantially benefitting from both a more meritorious initial GA population and from a higher number of IGA MUD iterations.

Recall that in Section III-A we pointed out that most of the existing detection techniques found in the open literature have not considered the so-called rank-deficient scenarios, where the number of users exceeds the number of the receiver antennas. For example, it is seen in Fig. 24 that the linear MMSE MUD suffered from a significant performance degradation, when the number of receiver antennas was fixed to $P=6$ and the number of users increased from $L=6$ to $L=8$, as a result of an insufficient degree of freedom for separating the different users in the rank-deficient scenarios. However, in such cases the BQMaided MMSE-IGA MUD [281], [282] was still capable of maintaining a near-ML performance. For instance, when we had $L=8$, the two-iteration based BQM-IGA MUD

\footnotetext{
${ }^{5}$ Note that in this case the associated complexity of the ML-aided scheme is as high as on the order of $O\left(2^{m L}\right)=O\left(2^{4 \cdot 6}\right)=O(16777216)$, which imposes an excessive complexity and, hence, cannot be simulated.
}

reduced the BER measured at $3 \mathrm{~dB}$ by four orders of magnitude in comparison to the MMSE-aided benchmark system, as evidenced by Fig. 24. This result characterizes the robustness of the BQM-IGA MUD, which has successfully suppressed the high MUI experienced in rank-deficient scenarios.

As an investigation on the GA's convergence characteristics, in Fig. 25 the performance of the $8 \times 6$ rankdeficient TTCM-aided MMSE-BQM-IGA-SDMA-OFDM system is illustrated, while using two IGA MUD iterations at a fixed $E_{b} / N_{0}$ value of $2 \mathrm{~dB}$. More specifically, at the lefthand side of Fig. 25 the population size $X$ was varied with the number of generations fixed at $Y=5$, while at the right-hand side of Fig. 25 the effect of a different number of generations $Y$ was evaluated at a fixed population size of $X=20$. Explicitly, as $X$ or $Y$ increases, a consistently reduced BER is observed, which approaches the optimum ML performance. Furthermore, we show in Fig. 26 the corresponding probability distribution function (PDF) curves of the IGA-aided system's BER performance using a similar configuration as that associated with Fig. 25, except that the population size was fixed at $X=40$. Each of the five PDFs was plotted based on the statistical distribution of the BER results generated by 300 independent simulation runs. As shown in Fig. 26, the peak of the PDF curve, which indicates the IGA-aided system's most likely attainable BER performance, is "shifted" closer to the average ML performance represented by the vertical dashed line seen at the left side of the figure, as the number of generations $Y$ increases. This fact demonstrates that the performance of the IGA MUD will converge to the

TTCM-MMSE-IGA-SDMA-OFDM, LX/P6, 4QAM, SWATM

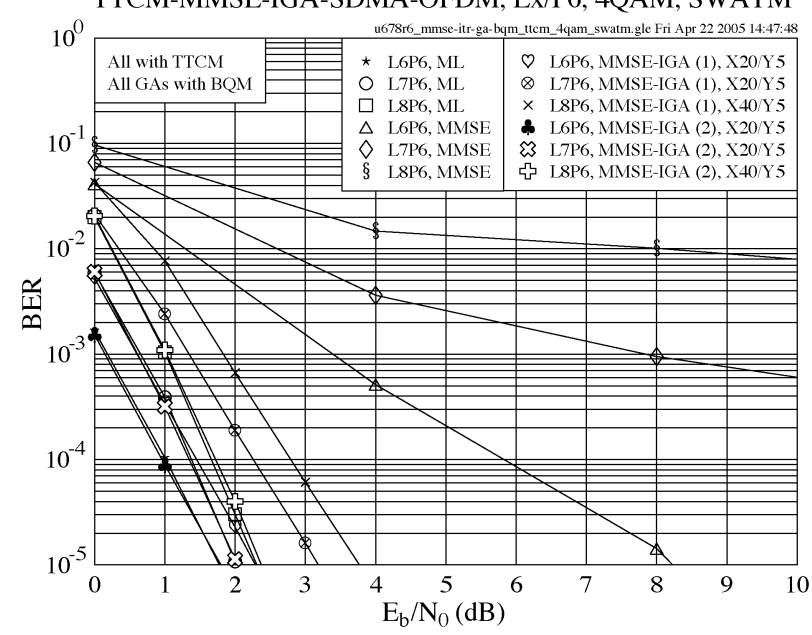

Fig. 24. BER versus $E_{b} / N_{O}$ performance comparison of the TTCM-assisted MMSE-IGA-SDMA-OFDM system using BQM, while employing a 4 QAM scheme for transmission over the SWATM channel, where $L=6,7,8$ users are supported with the aid of $P=6$ receiver antenna elements, respectively. 
TTCM-MMSE-IGA-SDMA-OFDM, L8/P6, 4QAM, SWATM

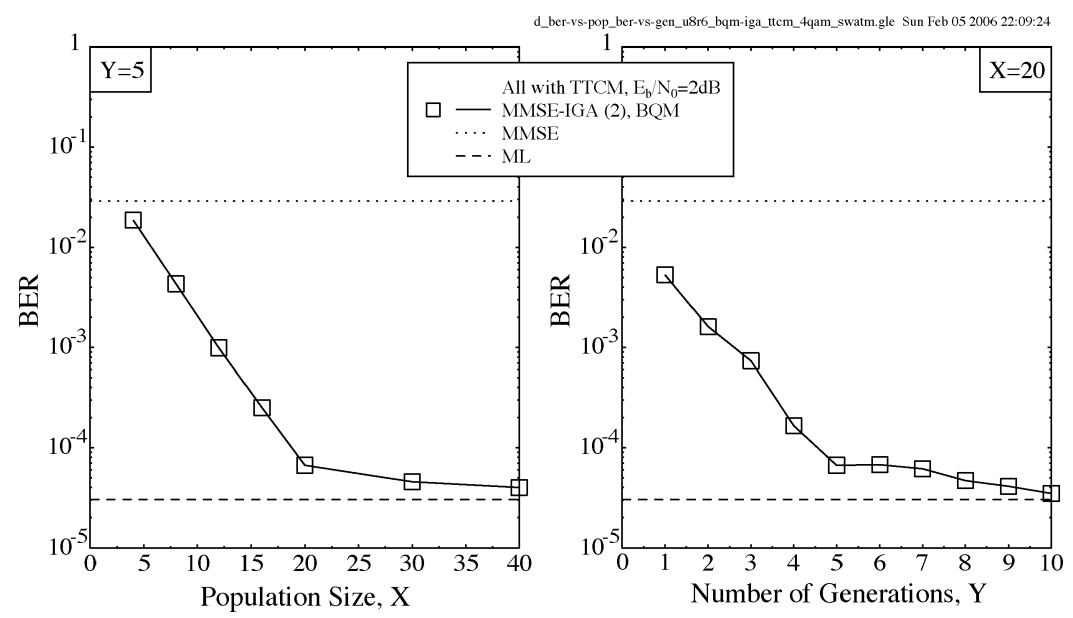

Fig. 25. BER versus $E_{b} / N_{0}$ performance of the TTCM-assisted MMSE-IGA-SDMA-OFDM system using BQM, while employing a 4 QAM scheme for transmission over the SWATM channel, where $L=8$ users are supported with the aid of $P=6$ receiver antenna elements. An $E_{b} / N_{0}$ value of $2 \mathrm{~dB}$ was assumed. Left: a variable population size $X$ with the number of generations fixed at $Y=5$; right: a different number of generations $Y$ with the population size fixed at $X=20$.

optimum one in rank-deficient scenarios, provided that a sufficiently high value of $Y$ is used. We also point out that in underloaded or fully loaded scenarios, a similar convergence having the same trend is expected.

In order to characterize the advantage of the BQM-IGA scheme in terms of the performance-versus-complexity tradeoff, in Table 13 we summarize the computational

TTCM-MMSE-IGA-SDMA-OFDM, L8/P6, 4QAM, SWATM

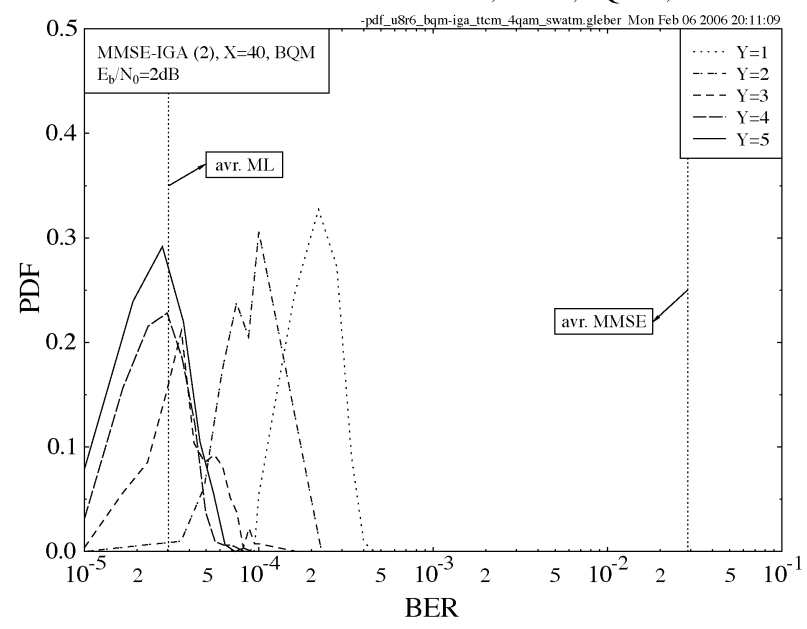

Fig. 26. PDF versus BER performance of the TTCM-assisted MMSE-IGA-SDMA-OFDM system using BQM, while employing a 4 QAM scheme for transmission over the SWATM channel, where $L=8$ users are supported with the aid of $P=6$ receiver antenna elements. An $E_{b} / N_{0}$ value of $2 \mathrm{~dB}$ was assumed. complexity imposed by the different MUDs assuming an $E_{b} / N_{0}$ value of $3 \mathrm{~dB}$, where the associated complexity was quantified in terms of the number of complex additions and multiplications imposed by the different MUDs on a per user basis. As observed in Table 13, the complexity of the ML MUD is significantly higher than that of the MMSE MUD or the IGA MUD, especially in highly rank-deficient scenarios. By contrast, the IGA MUD reduced the BER by up to five orders of magnitude in comparison to the MMSE MUD at a moderate complexity.

\section{GA-BASED DETECTION PROVIDING SOFT OUTPUTS}

GAs have demonstrated their power in both multiuser CDMA [25], [315], [316], [330], [331] and multiuser SDMA-OFDM [279]-[282] systems. However, all GAbased detection schemes found in the open literature were only capable of providing a hard-decision output for the channel decoder, which inevitably limits the system's achievable performance, until the recent emergence of the "soft" GAs [283], [284]. The GAs of [283], [284] benefit from their so-called population-based soft solutions and, hence, they are capable of outperforming their counterparts based on hard-decision outputs, while achieving a similar performance to that attained by soft-decision assisted optimum ML detection, especially in rankdeficient scenarios.

\section{A. Generating Soft Outputs}

In order to generate the soft information, the soft-bit value or log-likelihood ratio (LLR) associated with each bit 
Table 13 Comparison of MUD Complexity in Terms of Number of Complex Additions and Multiplications Measured at $E_{b} / N_{0}=3 \mathrm{~dB}$ on a Per User Basis in the 4 QAM TTCM-SDMA-OFDM System

\begin{tabular}{|c|c|c|c|c|c|c|c|c|c|}
\hline \multirow[b]{2}{*}{ MUD } & \multicolumn{3}{|c|}{$L=6$} & \multicolumn{3}{|c|}{$L=7$} & \multicolumn{3}{|c|}{$L=8$} \\
\hline & + & $x$ & BER & + & $x$ & BER & + & $x$ & BER \\
\hline ML & $2.8 \times 10^{4}$ & $2.7 \times 10^{4}$ & $1.8 \times 10^{-7}$ & $1.1 \times 10^{5}$ & $1.1 \times 10^{5}$ & $5.1 \times 10^{-7}$ & $4.3 \times 10^{5}$ & $4.2 \times 10^{5}$ & $8.5 \times 10^{-7}$ \\
\hline IGA & $8.1 \times 10^{2}$ & $7.9 \times 10^{2}$ & $2.2 \times 10^{-7}$ & $8.7 \times 10^{2}$ & $8.5 \times 10^{2}$ & $6.2 \times 10^{-7}$ & $1.8 \times 10^{3}$ & $1.7 \times 10^{3}$ & $9.8 \times 10^{-7}$ \\
\hline MMSE & $7.1 \times 10^{1}$ & $9.0 \times 10^{1}$ & $1.5 \times 10^{-3}$ & $7.1 \times 10^{1}$ & $8.8 \times 10^{1}$ & $7.5 \times 10^{-3}$ & $7.1 \times 10^{1}$ & $8.7 \times 10^{1}$ & $2.2 \times 10^{-2}$ \\
\hline
\end{tabular}

of each user's transmitted symbol at each OFDM subcarrier has to be calculated. This can be achieved by evaluating the GA's OF [283], [284]. More explicitly, in order to calculate the LLR of the $\left(m_{B}\right)$ th bit of the $l(l=1, \cdots, L)$ th user at the specific subcarrier considered, the $X$ number of individuals in the GA's final generation are divided into two groups, where the first (or second) group is constituted by those individuals that have a value of one (or zero) at the $\left(m_{B}\right)$ th bit of the lth user's estimated transmitted symbol. The resultant lowest OS calculated in each of the two groups is then compared to the average variance of the OSs denoted as $\omega$, and the smaller of the two will be used for calculating the corresponding LLR, which can, therefore, assist the channel decoder in improving the system's performance. Furthermore, the GA generating the above-mentioned populationbased soft outputs only imposes a modest complexity increase in comparison to the conventional hard-decision aided individual-based GAs [25], [279]-[282], [315]-[317], [330], [331]. This is because the only additional operation required by the proposed scheme is to compare $\omega$ to the OSs, which are already available, since the results of the OF evaluation carried out by the conventional GAs can be readily used.

\section{B. Example}

We consider a turbo convolutional (TC)-coded SDMassisted MIMO OFDM using soft GAs of [283], [284]. The soft GA's basic parameters were the same as those specified in Table 12, except that the mutation scheme was fixed to $\mathrm{BQM}$, while the population size and the number of generations were fixed to $X=500$ and $Y=5$, respectively. The specific TC code configuration and the parameters of the 8-path dispersive fading channel model of [332] used in the simulations are summarized in Table 14. Channel estimation was assumed to be perfect.

Fig. 27 characterizes the BER performance of the half-rate TC-aided QPSK GA-SDM-OFDM system employing both the conventional individual-based harddecoded GAs [25], [279]-[282], [315]-[317], [330], and the GA using population-based soft information [283], [284], respectively. For the sake of benchmarking, the BER performances of the systems using hard-decoded and/or soft-information aided MMSE and/or ML detec- tion are also provided. We had $m_{t}=8$ transmit antennas and $n_{r}=6$ receiver antennas for all schemes, implying a rank-deficient scenario having an effective throughput of $(2 \cdot 8 \cdot(1 / 2))=8$ BPS. As observed in Fig. 27, the MMSE-detected system suffered from a high error floor owing to the singular channel matrix associated with the rank-deficient scenario, while the system aided by the GA providing soft-outputs was capable of attaining an indistinguishable performance from that of the optimum soft-ML detected arrangement in the same scenario. Explicitly, an $E_{b} / N_{0}$ gain of about $2 \mathrm{~dB}$ was achieved by the GA using population-based soft outputs over its counterpart using the individual-based hard-decoded outputs.

\section{GA-AIDED JOINT CHANNEL ESTIMATION AND MULTIUSER DETECTION}

Apart from efficient multiuser detection, GAs can also be employed for channel estimation in wireless communications. More specifically, Yen and Hanzo [317] proposed a GA-aided multiuser CDMA single-antenna receiver, which jointly estimates the transmitted symbols and fading channel coefficients of all the users. A blind ML

Table 14 Basic Simulation Parameters Used in Section V-B

\begin{tabular}{||l|l|l|}
\hline \multirow{4}{*}{ TC code } & Modem & QPSK \\
\cline { 2 - 3 } & Code rate & $1 / 2$ \\
\cline { 2 - 3 } & Constraint length & 3 \\
\cline { 2 - 3 } & Octal generator polynomial & {$\left[\begin{array}{ll}7 \quad 5\end{array}\right]$} \\
\cline { 2 - 3 } & Turbo interleaver length & 124 bits \\
\hline \hline \multirow{4}{*}{ Channel } & CIRs & BUG [332] \\
\cline { 2 - 3 } & Paths & 8 \\
\cline { 2 - 3 } & Maximum path delay & $40 \mu \mathrm{s}$ \\
\cline { 2 - 3 } & Symbol duration & $160 \mu \mathrm{s}$ \\
\cline { 2 - 3 } & Subcarriers & 128 \\
\cline { 2 - 3 } & Channel interleaver length & 248 bits \\
\hline
\end{tabular}




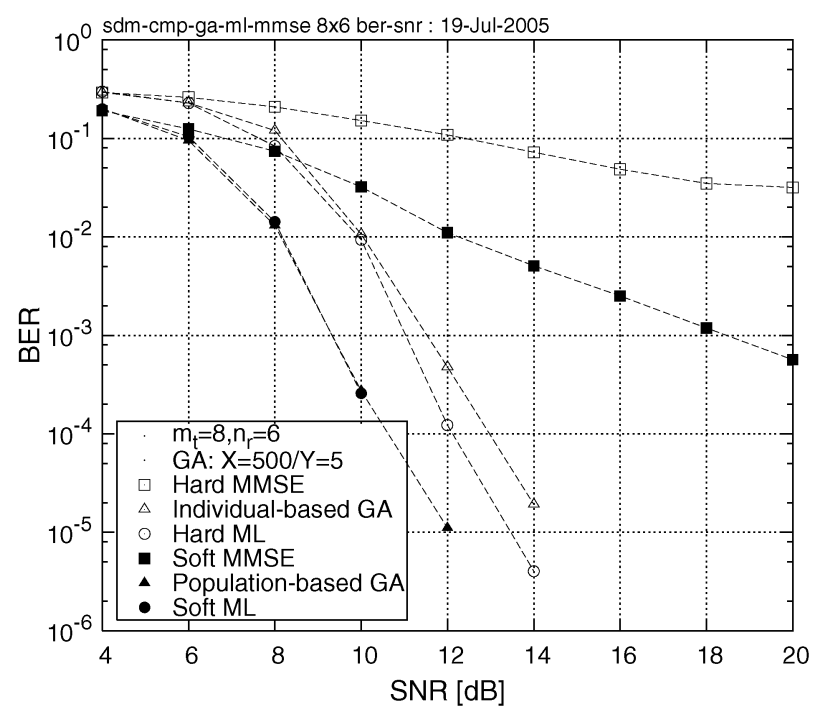

Fig. 27. BER performance comparison of the six-receiver TC-SDM-OFDM system using the individual-based hard-decoded and population-based soft-output GA detection, while employing a QPSK scheme having an effective throughput of 8 BPS.

equalization scheme based on concatenated channel and data estimation employing a micro genetic algorithm $(\mu \mathrm{GA})$ and the Viterbi algorithm (VA) was proposed in [333]. In [334] and [335], GA-based approaches were used for finding optimum training sequences for channel estimation in OFDM systems. However, as we have pointed out in Section III-B, in the open literature no channel estimation technique was proposed for rankdeficient BLAST or SDMA type multiuser MIMO OFDM systems until recently, when a new GA-assisted iterative joint channel estimation and multiuser detection (GAJCEMUD) approach [56], [324] emerged. In addition to the multiuser data symbols, the GA-JCEMUD technique of [56], [324] also incorporates the FD-CHTFs to be estimated for the MIMO-aided multiuser channel links into the overall genetic optimization process. Compared with the GA-aided pure MUDs [279]-[284], the GAJCEMUD does not result in an increase in computational complexity, since the latter exploits the same OF, namely that of (11) as the formers.

As an example of illustrating the performance of the GA-JCEMUD technique [56], [324], a visual comparison of the true and estimated FD-CHTFs of a two-path Rayleigh fading channel is portrayed in Fig. 28, where a pilot overhead of $\epsilon=5.0 \%$, an OFDM-symbol normalized Doppler frequency of $F_{D}=0.003$, and a $L \times P=4 \times 2$ rank-deficient scenario were assumed. More specifically, the $L=4$ users' FD-CHTFs associated with a specific receiver antenna element during a block of 40 consecutive OFDM symbols are plotted at a SNR value of $20 \mathrm{~dB}$. Each dot of the curves plotted in Fig. 28 represents a complexvalued FD-CHTF value at a specific subcarrier. By observing the perfect channel-knowledge based illustration at the top of Fig. 28, we can see that the FD-CHTF at each subcarrier evolves over the duration of the 40 OFDM symbols, where the thickness of the ring-shaped formations indicates the amount of FD-CHTF change during this time interval. The full perimeter of the ring is constituted by the $K=64$ spoke-like formations corresponding to the 64 OFDM subcarriers. Explicitly, the radii of the FD-CHTF rings associated with the four user-receiver channel links are significantly different. This is because each individual link is subjected to independent fading, and although the Doppler frequency encountered at the four links was assumed to be identical, their short-term envelope fluctuation observed over the 40 OFDM symbol durations
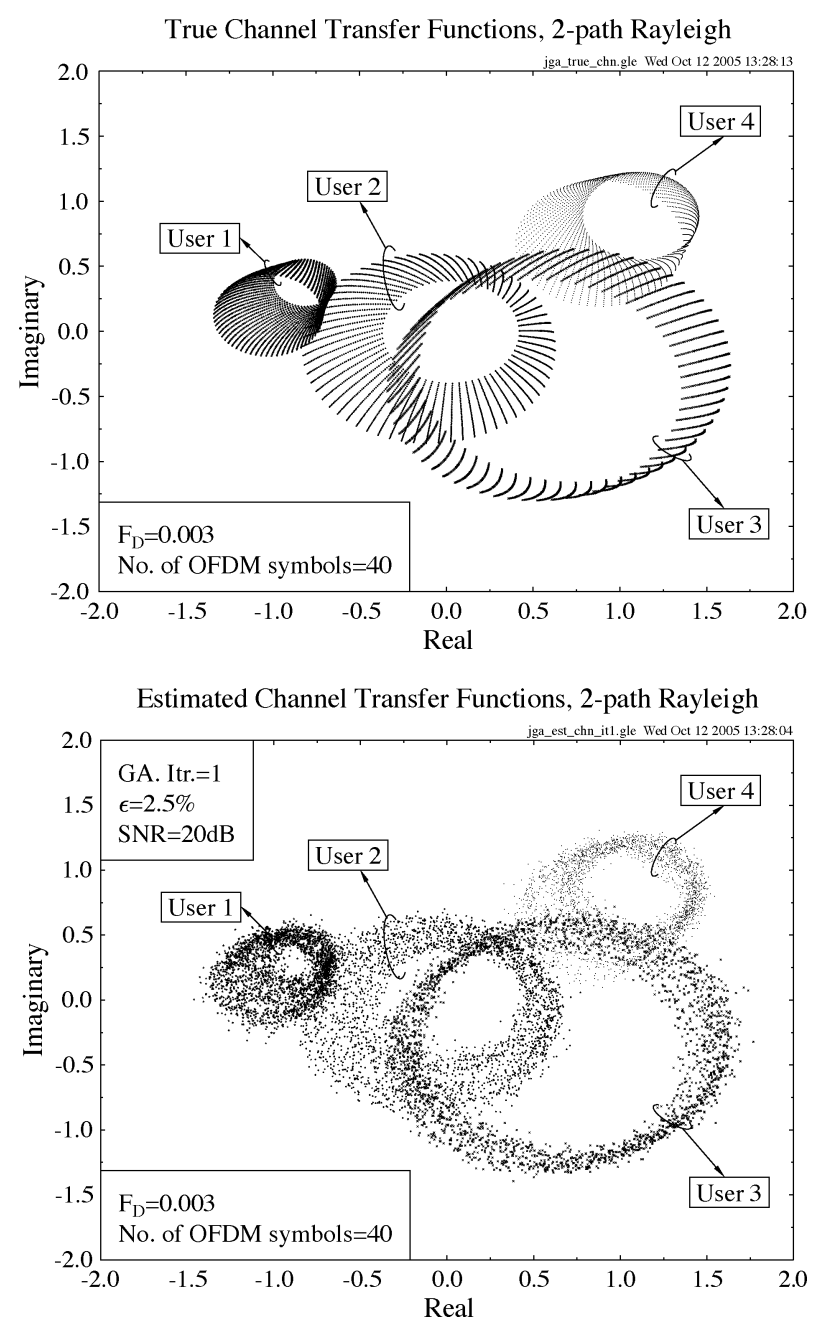

Fig. 28. Channel estimation performance of the iterative GA-JCEMUD assisted SDMA-OFDM system in the overloaded scenario, where $L=4$ users were supported with the aid of $P=2$ receiver antenna elements. The estimated FD-CHTFs $\hat{H}_{1}^{(l)}[n, k](I=1, \cdots, L ; k=1, \cdots, K)$ associated with $n=1, \cdots, 40$ consecutive OFDM symbols at the $p=1^{\text {st }}$ receiver antenna are plotted at a SNR value of $20 \mathrm{~dB}$, and compared with the true FD-CHTFs. 
is different. However, by comparing the subfigures at the top and bottom of Fig. 28, we can see that the FD-CHTF estimates closely match their true values, resulting in a similar FD-CHTF contour for each of the four channel links. This implies that the proposed GA-JCEMUD [56], [324] is capable of simultaneously capturing the fading envelope changes of each individual user-receiver link. Since an equally good performance was attained over all the userreceiver links, this fact demonstrates the global robustness of this approach in rank-deficient MIMO scenarios.

\section{SUMMARY AND CONCLUSION}

\section{A. Summary of Paper}

The combination of MIMOs and OFDM has emerged as a promising solution for future high-rate wireless communication systems. Our discourse commenced with a historical review of the 40-year OFDM literature in Section II. More specifically, the milestones in the history of OFDM were presented in Section II-A, where the key events and contributions across several decades were summarized in Tables 2-4. Furthermore, in Section II-B1 an overview of the advances in MIMO techniques was provided, followed by the introduction of combined MIMO OFDM systems in Section II-B2, where the associated contributions found in the literature were outlined in Tables 5-7. Moreover, in Section II-B3 we highlighted a subclass of MIMO arrangements, namely the SDMA-based techniques, summarizing the associated historical contributions in Tables 8 and 9 .

Based on the state-of-the-art review of Section II, in Section III we further discussed the specific limitations of existing techniques designed for multiuser MIMO OFDM systems. More specifically, in Section III-A and Section III-B we focussed on the detection techniques and the channel estimation approaches, respectively. Explicitly, we pointed out that in the open literature there was a paucity of information on multiuser MIMO OFDM detectors, which are capable of supporting a higher number of users than the number of receiver antennas. Recall that in such rank-deficient scenarios a particular challenge is imposed by the insufficient degree of freedom owing to having a singular channel matrix. These challenging difficulties, however, may be overcome with the advent of the advocated GA-assisted hybrid techniques. Originating from the evolutionary computing research community, GAs have shown their compelling power in many computationally demanding applications [27]-[31]. Furthermore, they can also be applied to MIMO OFDM systems, resulting in attractive and efficient solutions to the above-mentioned problems.

Firstly, the GA MUDs [279]-[282] introduced in Section IV have exhibited an excellent performance versus complexity tradeoff by providing a similar performance to that attained by the optimum ML MUD at a significantly lower computational complexity, especially at high user loads. Moreover, the combined employment of $\mathrm{BQM}$ and the channel decoder aided iterative detection framework [281], [282] is capable of further enhancing the GA MUD's achievable performance. For example, in the low-throughput fully loaded six-user 4 QAM MIMO SDMA-OFDM system of Fig. 22, a twoiteration BQM-IGA MUD associated with a population size of $X=20$ and $Y=5$ generations was capable of achieving the same performance as the optimum MLaided system at a complexity of 200 OF evaluations, which is only about $5 \%$ of the MUD-related complexity imposed by the optimum ML MUD. On the other hand, in high-throughput six-user systems employing for example a 16 QAM modem, a two-iteration BQM-IGA MUD associated with $X=40$ and $Y=5$ achieved an $E_{b} / N_{0}$ gain of about $7 \mathrm{~dB}$ over the MMSE MUD benchmarker at the BER of $10^{-5}$, as observed in Fig. 23. Furthermore, the associated $E_{b} / N_{0}$ gain was attained at a modest complexity of 400 OF evaluations, which is only $0.00238 \%$ of the excessive complexity imposed by the ML MUD that cannot be simulated in this case. In addition, the BQM-IGA MUD is capable of providing a near-optimum performance even in the so-called rankdeficient scenarios, while many conventional detection techniques suffer from an excessively high error floor. In the example of Fig. 24, where we had $L=8$ users and $P=6$ receivers, the two-iteration based BQM-IGA MUD reduced the BER recorded at an $E_{b} / N_{0}$ value of $3 \mathrm{~dB}$ by four orders of magnitude in comparison to the classic MMSE MUD aided benchmarker system. The BER PDF curves in Fig. 26 also demonstrated the GA's robust convergence in terms of MUI suppression. Fig. 17 and Table 13 further illustrated the performance versus complexity benefits of the GA-based MUDs.

With the aid of the GAs' population-based soft outputs [283], [284] introduced in Section V, the performance of the channel coded MIMO OFDM systems using conventional hard-decision aided individual-based GAs [25], [279]-[282], [315]-[317], [330], [331] can be improved at the cost of a modest complexity increase. For example, in the TC-coded rank-deficient SDM-OFDM system characterized in Fig. 27, it was shown that the performance of the currently known GA-assisted systems can be improved by about $2 \mathrm{~dB}$ with the aid of the GA's soft solution, approaching the optimum performance of the soft-information assisted ML detector.

Finally, in Section VI we reviewed the GA-assisted iterative joint channel estimation and symbol detection approach of [56], [324], which provides an efficient solution to the challenging channel estimation problem in rank-deficient multiuser MIMO OFDM systems. Benefitting from its inherent genetically guided optimization, the GA-JCEMUD of Section VI exhibits a robust performance even in rank-deficient scenarios, as exemplified in Fig. 28. 


\section{B. Future Research}

Based on the solutions discussed throughout this paper, we demonstrated a number of significant benefits brought about by the GAs for wireless communications. It is worth pointing out that the proposed GAs may be further improved in various ways. For example, the value of the mutation probability can be adapted according to the number of users and/or the GA's generation index. Furthermore, the GA's population-based soft output [283], [284] can be improved, if the OSs of all meritorious individuals are stored throughout all generations, which may be used for improving the reliability of the GA's soft output. Additionally, the GA individual's symbol chromosome, which consists of the multiple users' hard-decoded symbol estimates, may also be represented by the soft bit estimates, enabling the GA to benefit from the soft information provided by the channel decoders during the external iterative processing. This is expected to improve the performance of the iterative GAs, such as those used in the IGA MUD of [281], [282] and in the GA-JCEMUD of [56], [324]. Moreover, the joint channel estimation and symbol detection approach of [56], [324] can also be further enhanced by introducing a soft-input soft-output mechanism. More specifically, not only the GA-optimized FD-CHTF estimates, but also the GA-optimized symbol estimates can be forwarded to the first-stage MUD for assisting in the initial symbol detection invoked in the next iteration.
Generally speaking, GAs belong to the family of evolutionary algorithms (EAs) [336], [337], which invokes the principles of natural evolution. With the aid of the recent advances in artificial intelligence (AI), a range of other problem-solving methods have also emerged. One of these techniques is constituted by the family of neural networks (NNs) [338], [339], which is based on the models that mimick the operation of how biological neurons are connected in the human brain. Specifically, NNs are also applicable to the field of multiuser detection. For example, the so-called radial basis function (RBF) [340]-[342] based NNs have been proposed for multiuser detection in CDMA type systems [343]-[345]. However, hardly any research has been conducted in the context of RBF-assisted multiuser OFDM systems [346]. Explicitly, exploiting RBF with or without the aid of EAs/GAs for employment in OFDM, MIMO-OFDM, and SDMA-OFDM systems constitutes a promising novel research area.

\section{Acknowledgment}

The work reported in this paper has formed part of the Wireless Enablers 2.2 of the Core 3 Research Programme of the Virtual Centre of Excellence in Mobile and Personal Communications, Mobile VCE, www.mobilevce.com, whose funding support, including that of EPSRC, is gratefully acknowledged. Fully detailed technical reports on this research are available to Industrial Members of Mobile VCE.

\section{REFERENCES}

[1] L. Hanzo, M. Münster, B. J. Choi, and T. Keller, OFDM and MC-CDMA for Broadband Multi-User Communications, WLANs and Broadcasting. Piscataway, NJ: IEEE Press/Wiley, 2003.

[2] L. Hanzo, S. X. Ng, T. Keller, and W. T. Webb, Quadrature Amplitude Modulation: From Basics to Adaptive Trellis-Coded, Turbo-Equalised and Space-Time Coded OFDM, CDMA and MC-CDMA Systems, 3rd ed. Piscataway, NJ: IEEE Press/Wiley, 2004.

[3] R. V. Nee and R. Prasad, OFDM for Wireless Multimedia Communications. London, U.K.: Artech House, 2000.

[4] J. A. C. Bingham, "Multicarrier modulation for data transmission: An idea whose time has come," IEEE Commun. Mag., vol. 28, no. 5, pp. 5-14, May 1990.

[5] W. C. Chung, N. J. Aug., and D. S. Ha, "Signaling and multiple access techniques for ultra wideband 4G wireless communication systems," Wireless Commun., vol. 12, pp. 46-55, Apr. 2005.

[6] L. Hanzo, T. H. Liew, and B. L. Yeap, Turbo Coding, Turbo Equalisation and Space-Time Coding for Transmission Over Fading Channels. Piscataway, NJ: IEEE Press/Wiley, 2002.

[7] J. H. Winters, "Optimum combining in digital mobile radio with cochannel interference," IEEE J. Sel. Areas Commun., vol. SAC-2, no. 4, pp. 528-539, Jul. 1984.

[8] J. H. Winters, "On the capacity of radio communication systems with diversity in a Rayleigh fading environment," IEEE J. Sel. Areas Commun., vol. SAC-5, no. 5, pp. 871-878, Jun. 1987.

[9] S. Ng and L. Hanzo, "On the MIMO channel capacity of multi-dimensional signal sets," in Proc. 60th IEEE Conf. Vehicular Technology, Sep. 2006, vol. 3, pp. 1594-1598.

[10] G. J. Foschini, "Layered space-time architecture for wireless communication in a fading environment when using multi-element antennas," Bell Labs Tech. J., vol. Autumn, pp. 41-59, Oct. 1996.

[11] S. M. Alamouti, "A simple transmit diversity technique for wireless communications," IEEE J. Sel. Areas Commun., vol. 16, no. 8 pp. 1451-1458, Oct. 1998.

[12] V. Tarokh, A. Naguib, N. Seshadri, and A. R. Calderbank, "Space-time codes for high data rate wireless communication: Performance criteria in the presence of channel estimation errors, mobility, and multile paths," IEEE Trans. Commun., vol. 47, no. 2, pp. 199-207, Feb. 1999.

[13] T. Liew and L. Hanzo, "Space-time block codes and concatenated channel codes for wireless communications," Proc. IEEE, vol. 90, no. 2, pp. 187-219, Feb. 2002.
[14] T.-H. Liew and L. Hanzo, "Space-time trellis and space-time block coding versus adaptive modulation and coding aided OFDM for wideband channels," IEEE Trans. Veh. Technol., vol. 55, no. 1, pp. 173-187, Jan. 2006.

[15] J. Blogh and L. Hanzo. (2002). Third-Generation Systems and Intelligent Networking. Piscataway, NJ, IEEE Press/Wiley. [Online]. Available: http:// www-mobile.ecs.soton.ac.uk

[16] R. W. Chang, "Synthesis of band-limited orthogonal signals for multichannel data transmission," Bell System Tech. J., vol. 45 , pp. 1775-1796, Dec. 1966.

[17] European Telecommunication Standard Institute, Digital Audio Broadcasting (DAB); $D A B$ to Mobile, Portable and Fixed Receivers, ETSI ETS 300401 ed.1, Feb. 1995.

[18] European Telecommunication Standard Institute, Digital Video Broadcasting (DVB); Framing Structure, Channel Coding and Modulation for Digital Terrestrial Television (DVB-T), ETSI ETS 300744 ed.1, Mar. 1997.

[19] European Telecommunication Standard Institute, Digital Video Broadcasting (DVB); Transmission System for Handheld Terminals (DVB-H), ETSI EN 302304 V1.1.1, Nov. 2004.

[20] European Telecommunication Standard Institute, Radio Equipment and Systems (RES); HIgh PErformance Radio Local Area 
Network (HIPERLAN) Type 1; Functional Specification, ETSI ETS 300652 ed.1, Oct. 1996.

[21] M. Münster and L. Hanzo, "Parallel interference cancellation assisted decision-directed channel estimation for OFDM systems using multiple transmit antennas," IEEE Trans. Wireless Commun., vol. 4, no. 5, pp. 2148-2162, Sep. 2005.

[22] V. Tarokh, H. Jafarkhani, and A. R. Calderbank, "Space-time block codes from orthogonal designs," IEEE Trans. Inf. Theory, vol. 45, no. 5 , pp. 1456-1467, May 1999.

[23] T. Liew, B. Yeap, C. Wong, and L. Hanzo, "Turbo coded adaptive modulation versus space-time trellis codes for transmission over dispersive channels," IEEE Trans. Wireless Commun., vol. 3, no. 6, pp. 2019-2029, Nov. 2004.

[24] B. L. Yeap and L. Hanzo, "Reduced complexity I/Q turbo detection for space-time trellis coded systems," IEEE Trans. Veh. Technol., vol. 53, no. 4, pp. 278-1286, Jul. 2004.

[25] L. Hanzo, L.-L. Yang, E.-L. Kuan, and K. Yen, Single- and Multi-Carrier DS-CDMA: Multi-User Detection, Space-Time Spreading, Synchronisation and Standards. Piscataway, NJ: IEEE Press/Wiley, 2003.

[26] L.-L. Yang and L. Hanzo, "Performance of broadband multicarrier DS-CDMA using space-time spreading assisted transmit diversity," IEEE Trans. Wireless Commun., vol. 4, no. 3, pp. 885-894, May 2005.

[27] J. Holland, Adaptation in Natural and Artificial Systems. Ann Arbor, MI: Univ. Michigan Press, 1975.

[28] D. E. Goldberg, Genetic Algorithms in Search, Optimization, and Machine Learning. Reading, MA: Addison-Wesley, 1989.

[29] M. Mitchell, An Introduction to Genetic Algorithms. Cambridge, MA: MIT Press, 1996.

[30] D. Whitley, "A genetic algorithm tutorial," Statist. Computing, vol. 4, pp. 65-85, Jun. 1994.

[31] S. Forrest, "Genetic algorithms: Principles of natural selection applied to computation," Science, vol. 261, pp. 872-878, Aug. 1993.

[32] J. L. Holsinger, "Digital communication over fixed time-continuous channels with memory-With special application to telephone channels," MIT-Lincoln Lab., Cambridge, MA, Tech. Rep. 366, Oct. 1964.

[33] A Multicarrier Primer, ANSI T1E1.4/91-157, Nov. 1991, J. M. Cioffi.

[34] ANSI Committee T1-Telecommunications, "A technical report on high-bit-rate digital subscriber lines (HDSL)," Tech. Rep. 28, Feb. 1994.

[35] Very-High-Speed Digital Subscriber Lines: System Requirements, ANSI T1E1.4 VDSL SR: 98-043R5, Sep. 1998.

[36] European Telecommunication Standard Institute, Transmission and Multiplexing (TM); Access Transmission Systems on Metallic Access Cables; Very High Speed Digital Subscriber Line (VDSL); Part 1: Functional requirements, ETSI TS 101 270-1 V1.1.2, Jun. 1998.

[37] European Telecommunication Standard Institute, Broadband Radio Access Networks (BRAN); Inventory of Broadband Radio Technologies and Techniques, ETSI TR 101173 V1.1.1, May 1998.

[38] Institute of Electrical and Electronics Engineers, IEEE Standard 802.11a: Wireless
LAN Medium Access Control (MAC) and Physical Layer (PHY) Specifications: High-Speed Physical Layer in the $5 \mathrm{GHz}$ Band, 1999.

[39] Institute of Electrical and Electronics Engineers, IEEE Standard 802.11: Wireless Lan Medium Access Control (MAC) and Physical Layer (PHY) Specifications, Nov. 18, 1997.

[40] Institute of Electrical and Electronics Engineers, IEEE Standard 802.11g: Wireless LAN Medium Access Control (MAC) and Physical Layer (PHY) Specifications 2003.

[41] Institute of Electrical and Electronics Engineers, IEEE Candidate Standard 802.11n: Wireless LAN Medium Access Control (MAC) and Physical Layer (PHY) Specifications, 2004. [Online]. Available: http:/grouper.ieee.org/groups/802/11/ Reports/tgn_update.htm

[42] Institute of Electrical and Electronics Engineers, IEEE Standard 802.16: Air Interface for Fixed Broadband Wireles Access Systems, 2004.

[43] B. R. Saltzberg, "Performance of an efficient parallel data transmission system," IEEE Trans. Commun., vol. COM-15, no. 6 , pp. 805-811, Dec. 1967.

[44] R. W. Chang, "Orthogonal frequency division multiplexing," U.S. Patent 3488 445, filed Nov. 14, 1966, issued Jan. 6, 1970.

[45] S. B. Weinstein and P. M. Ebert, "Data transmission by frequency-division multiplexing using the Discrete Fourier Transform," IEEE Trans. Commun., vol. COM-19, no. 5, pp. 628-634, Oct. 1971.

[46] B. Hirosaki, "An analysis of automatic equalizers for orthogonally multiplexed QAM systems," IEEE Trans. Commun., vol. COM-28, no. 1, pp. 73-83, Jan. 1980.

[47] W. E. Keasler, D. L. Bitzer, and P. T. Tucker, "High-speed modem suitable for operating with a switched network," U.S. Patent 4206 320, filed Aug. 21, 1978, issued Jun. 3, 1980

[48] L. J. Cimini, Jr., "Analysis and simulation of a digital mobile channel using orthogonal frequency division multiplexing," IEEE Trans. Commun., vol. COM-33, no. 7 , pp. 665-675, Jul. 1985.

[49] M. Alard and R. Lassalle, "Principles of modulation and channel coding for digital broadcasting for mobile receivers," EBU Tech. Rev., pp. 168-190, Aug. 1987.

[50] M. Yabusaki, "Asia pacific viewpoint and activities: Introduction," presented at the 4G Forum, London, U.K., May 27, 2003.

[51] R. W. Chang and R. A. Gibby, "A theoretical study of performance of an orthogonal multiplexing data transmission scheme," IEEE Trans. Commun., vol. COM-16, no. 4 pp. 529-540, Aug. 1968.

[52] A. Peled and A. Ruiz, "Frequency domain data transmission using reduced computational complexity algorithms," in Proc. 1980 IEEE Int. Conf. Acoustics, Speech, and Signal Processing (ICASSP '80), Denver, CO, Apr. 9-11, 1980, vol. 5, pp. 964-967.

[53] B. Hirosaki, "An orthogonally multiplexed QAM system using the discrete fourier transform," IEEE Trans. Commun., vol. 29, no. 7, pp. 982-989, Jul. 1981

[54] B. Hirosaki, S. Hasegawa, and A. Sabato, "Advanced groupband data modem using orthogonally multiplexed QAM technique,"
IEEE Trans. Commun., vol. COM-34, no. 6, pp. 587-592, Jun. 1986.

[55] I. Kalet, "The multitone channel," IEEE Trans. Commun., vol. 37, no. 2, pp. 119-124, Feb. 1989.

[56] L. Hanzo and T. Keller, An OFDM and MC-CDMA Primer. Piscataway, NJ: IEEE Press/Wiley, 2006.

[57] Z. Wang and G. B. Giannakis, "Wireless multicarrier communications," Signal Process. Mag., pp. 29-48, May 2000.

[58] T. Keller and L. Hanzo, "Adaptive multicarrier modulation: A convenient framework for time-frequency processing in wireless communications," Proc. IEEE, vol. 88, no. 5, pp. 611-640, May 2000.

[59] L. Hanzo, B. J. Choi, and M. Münster, "A stroll along multi-carrier boulevard towards next-generation plaza-OFDM background and history," IEEE Veh. Technol. Soc. Newslett., vol. 51, pp. 4-10, Nov. 2004.

[60] L. Hanzo, B. J. Choi, and M. Münster, "A stroll along multi-carrier boulevard towards next-generation plaza-Space-time coded adaptive OFDM and MC-CDMA comparison," IEEE Veh. Technol. Soc. Newslett., vol. 51, pp. 10-19, Nov. 2004.

[61] R. Steele and L. Hanzo, Mobile Radio Communications: Second and Third Generation Cellular and WATM Systems, 2nd ed. Piscataway, NJ: IEEE Press/ Wiley, 1999.

[62] A. J. Viterbi, CDMA: Principles of Spread Spectrum Communication. Reading, MA Addison-Wesley, 1995.

[63] K. S. Zigangirov, Theory of Code Division Multiple Access Communication. Piscataway, NJ: IEEE Press/Wiley, 20040-471-45712-4.

[64] L. E. Miller and J. S. Lee, CDMA Systems Engineering Handbook. London, U.K.: Artech House, 1998.

[65] J. S. Lee, "Overview of the technical basis of Qualcomm's CDMA cellular telephone system design: A view of North American TIA/EIA IS-95," in Proc. 1994 Int. Conf. Communications Systems (ICCS '94), Singapore, Nov. 14-18, 1994, vol. 2, pp. 353-358.

[66] I. Koffman and V. Roman, "Broadband wireless access solutions based on OFDM access in IEEE 802.16," IEEE Commun. Mag., vol. 40, no. 4, pp. 96-103, Apr. 2002.

[67] R. Laroia, S. Uppala, and J. Li, "Designing a mobile broadband wireless access network," IEEE Signal Process. Mag., vol. 21, no. 5, pp. 20-28, Sep. 2004

[68] P. Xia, S. Zhou, and G. B. Giannakis, "Bandwidth- and power-efficient multicarrier multiple access," IEEE Trans. Commun., vol. 51, no. 11, pp. 1828-1837, Nov. 2003

[69] Z. Cao, U. Tureli, and Y. Yao, "Deterministic multiuser carrier-frequency offset estimation for interleaved OFDMA uplink," IEEE Trans. Commun., vol. 52, no. 9 , pp. 1585-1594, Sep. 2004.

[70] R. Bercovich, "OFDM enhances the 3G high-speed data access," in Proc. GSPx 2004 Conf., Santa Clara, CA, Sep. 27-30, 2004. [Online]. Available: http://www.techonline. com/pdf/pavillions/gspx/2004/1084.pdf

[71] T. May, H. Rohling, and V. Engels, "Performance analysis of Viterbi decoding for 64-DAPSK and 64-QAM modulated OFDM signals," IEEE Trans. Commun. vol. 46, no. 2, pp. 182-190, Feb. 1998.

[72] L. Lin, L. J. Cimini, Jr., and C.-I. Chuang, "Comparison of convolutional and turbo 
codes for OFDM with antenna diversity in high-bit-rate wireless applications," IEEE Commun. Lett., vol. 4, no. 9, pp. 277-279, Sep. 2000.

[73] W. D. Warner and C. Leung, "OFDM/FM frame synchronization for mobile radio data communication," IEEE Trans. Veh. Technol., vol. 42, no. 3, pp. 302-313, Aug. 1993.

[74] P. H. Moose, "A technique for orthogonal frequency division multiplexing frequency offset correction," IEEE Trans. Commun., vol. 42, no. 10, pp. 2908-2914, Oct. 1994.

[75] T. Pollet, M. V. Bladel, and M. Moeneclaey, "BER sensitivity of OFDM systems to carrier frequency offset and Wiener phase noise," IEEE Trans. Commun., vol. 43, no. 234, pp. 191-193, Feb./Mar./Apr. 1995.

[76] A. E. Jones, T. A. Wilkinson, and S. K. Barton, "Block coding scheme for reduction of peak to mean envelope power ratio of multicarrier transmission schemes," Electron. Lett., vol. 30, pp. 2098-2099, Dec. 1994.

[77] S. J. Shepherd, P. W. J. V. Eetvelt, C. W. Wyatt-Millington, and S. K. Barton, "Simple coding scheme to reduce peak factor in QPSK multicarrier modulation," Electron. Lett., vol. 31, pp. 1131-1132, Jul. 1995.

[78] D. Wulich, "Reduction of peak to mean ratio of multicarrier modulation using cyclic coding," Electron. Lett., vol. 32, pp. 432-433, Feb. 1996.

[79] D. Wulich, "Peak factor in orthogonal multicarrier modulation with variable levels," Electron. Lett., vol. 32, pp. 1859-1861, Sep. 1996.

[80] X. Li and L. J. Cimini, Jr., "Effects of clipping and filtering on the performance of OFDM," in Proc. 1997 IEEE 47th Vehicular Technology Conf. (VTC '97 Spring), Phoenix, AZ, May 4-7, 1997, vol. 3, pp. 1634-1638.

[81] X. Li and L. J. Cimini, Jr., "Effects of clipping and filtering on the performance of OFDM," IEEE Commun. Lett., vol. 2, no. 5, pp. 131-133, May 1998.

[82] S. Hara and R. Prasad, "Overview of multicarrier CDMA," IEEE Commun. Mag., vol. 35, no. 12, pp. 126-133, Dec. 1997.

[83] Y. Li, L. J. Cimini, Jr., and N. R. Sollenberger, "Robust channel estimation for OFDM systems with rapid dispersive fading channels," IEEE Trans. Commun., vol. 46, no. 7, pp. 902-915, Jul. 1998.

[84] Y. Li and N. R. Sollenberger, "Adaptive antenna arrays for OFDM systems with cochannel interference," IEEE Trans. Commun., vol. 47, no. 2, pp. 217-229, Feb. 1999.

[85] S. Armour, A. Nix, and D. Bull, "Pre-FFT equaliser design for OFDM," Electron. Lett., vol. 35, pp. 539-540, Apr. 1999.

[86] S. Armour, A. Nix, and D. Bull, "Performance analysis of a pre-FFT equalizer design for DVB-T," IEEE Trans. Consumer Electron., vol. 45, no. 3, pp. 544-552, Aug. 1999.

[87] S. Armour, A. Nix, and D. Bull, "Complexity evaluation for the implementation of a pre-FFT equalizer in an OFDM receiver," IEEE Trans. Consumer Electron., vol. 46, no. 3, pp. 428-437, Aug. 2000.

[88] B. Y. Prasetyo and A. H. Aghvami, "Simplified frame structure for MMSE-based fast burst synchronisation in OFDM systems," Electron. Lett., vol. 35, pp. 617-618, Apr. 1999.
[89] B. Y. Prasetyo, F. Said, and A. H. Aghvami, "Fast burst synchronisation technique for OFDM-WLAN systems," Inst. Electr. Eng. Proc.-Communications, vol. 147, pp. 292-298, Oct. 2000.

[90] P. Cherriman, T. Keller, and L. Hanzo, "Orthogonal frequency-division multiplex transmission of H.263 encoded video over highly frequency-selective wireless networks," IEEE Trans. Circuits Syst. Video Technol., vol. 9, no. 5, pp. 701-712, Aug. 1999.

[91] C. Y. Wong, R. S. Cheng, K. B. Lataief, and R. D. Murch, "Multiuser OFDM with adaptive subcarrier, bit, and power allocation," IEEE J. Sel. Areas Commun., vol. 17, no. 10, pp. 1747-1758, Oct. 1999.

[92] K. Fazel and G. Fettweis, Multi-carrier Spread Spectrum and Related Topics. Norwell, MA: Kluwer, 20000-7923-9973-0.

[93] C.-S. Lee, T. Keller, and L. Hanzo, "OFDM-based turbo-coded hierarchical and non-hierarchical terrestrial mobile digital video broadcasting," IEEE Trans. Broadcast., vol. 46 , no. 1, pp. 1-22, Mar. 2000.

[94] T. Keller and L. Hanzo, "Adaptive modulation techniques for duplex OFDM transmission," IEEE Trans. Veh. Technol., vol. 49, no. 5, pp. 1893-1906, Sep. 2000

[95] T. Keller, L. Piazzo, P. Mandarini, and L. Hanzo, "Orthogonal frequency division multiplex synchronization techniques for frequency-selective fading channels," IEEE J. Sel. Areas Commun., vol. 19, no. 6, pp. 999-1008, Jun. 2001.

[96] B. Lu, X. Wang, and K. R. Narayanan, "LDPC-based space-time coded OFDM systems over correlated fading channels: Performance analysis and receiver design," in Proc. 2001 IEEE Int. Symp. Information Theory (ISIT '01), Jun. 24-29, 2001, vol. 1, p. 313.

[97] B. Lu, X. Wang, and K. R. Narayanan, "LDPC-based space-time coded OFDM systems over correlated fading channels: Performance analysis and receiver design," IEEE Trans. Commun., vol. 50, no. 1, pp. 74-88, Jan. 2002.

[98] B. Lu, X. Wang, and Y. Li, "Iterative receivers for space-time block coded OFDM systems in dispersive fading channels," in Proc. 2001 IEEE Global Telecommunications Conf. (GLOBECOM '01), Nov. 25-29, 2001, vol. 1, pp. 514-518.

[99] B. Lu, X. Wang, and Y. Li, "Iterative receivers for space-time block-coded OFDM systems in dispersive fading channels," IEEE Trans. Wireless Commun., vol. 1, no. 2, pp. 213-225, Apr. 2002.

[100] P. J. Cherriman, T. Keller, and L. Hanzo, "Subband-adaptive turbo-coded OFDM-based interactive video telephony," IEEE Trans. Circuits Syst. Video Technol., vol. 12, no. 10, pp. 829-839, Oct. 2002.

[101] O. Simeone, Y. Bar-Ness, and U. Spagnolini, "Pilot-based channel estimation for OFDM systems by tracking the delay-subspace," IEEE Trans. Wireless Commun., vol. 3, no. 1, pp. 315-325, Jan. 2004.

[102] J. Zhang, H. Rohling, and P. Zhang, "Analysis of ICI cancellation scheme in OFDM systems with phase noise," IEEE Trans. Broadcast., vol. 50, no. 2, pp. 97-106, Jun. 2004.

[103] M. C. Necker and G. L. Stüber, “Totally blind channel estimation for OFDM on fast varying mobile radio channels," IEEE Trans. Wireless Commun., vol. 3, no. 5, pp. 1514-1525, Sep. 2004.
[104] A. Doufexi, S. Armour, A. Nix, P. Karlsson, and D. Bull, "Range and throughput enhancement of wireless local area networks using smart sectorised antennas," IEEE Trans. Wireless Commun., vol. 3, no. 5, pp. 1437-1443, Sep. 2004.

[105] E. Alsusa, Y. Lee, and S. McLaughlin, "Channel-adaptive sectored multicarrier packet based systems," Electron. Lett., vol. 40, pp. 1194-1196, Sep. 2004.

[106] C. Williams, M. A. Beach, and S. McLaughlin, "Robust OFDM timing synchronisation," Electron. Lett., vol. 41 , pp. 751-752, Jun. 2005.

[107] J. H. Winters, "Wireless PBX/LAN system with optimum combining," U.S. Patent 4639 914, filed Dec. 6, 1984, issued Jan. 27, 1987.

[108] J. H. Winters, "Optimum combining for indoor radio systems with multiple users," IEEE Trans. Commun., vol. COM-35, no. 11, pp. 1222-1230, Nov. 1987.

[109] J. Salz, "Digital transmission over cross-coupled linear channels," AT\&T Tech. J., vol. 64, pp. 1147-1159, Jul.-Aug. 1985.

[110] S. Cheng and S. Verdu, "Gaussian multiaccess channels with ISI: Capacity region and multiuser water-filling," IEEE Trans. Inf. Theory, vol. 39, no. 3, pp. 773-785, May 1993.

[111] A. Duel-Hallen, “Equalizers for multiple input/multiple output channels and PAM systems with cyclostationary input sequences," IEEE J. Sel. Areas Commun., vol. 10, no. 3, pp. 630-639, Apr. 1992.

[112] J. H. Winters, J. Salz, and R. D. Gitlin, "The impact of antenna diversity on the capacity of wireless communication systems," IEEE Trans. Commun., vol. 5, no. 234, pp. 1740-1751, Feb./Mar./Apr. 1994.

[113] J. Yang and S. Roy, "On joint transmitter and receiver optimization for multiple-input multiple-output (MIMO) transmission systems," IEEE Trans. Commun., vol. 42, no. 12, pp. 3221-3231, Dec. 1994.

[114] J. Yang and S. Roy, "Joint transmitter-receiver optimization for multi-input multi-output systems with decision feedback," IEEE Trans. Inf. Theory, vol. 40, no. 5, pp. 1334-1347, Sep. 1994.

[115] J. H. Winters, "The diversity gain of transmit diversity in wireless systems with Rayleigh fading," IEEE Trans. Veh. Technol., vol. 47, no. 1, pp. 119-123, Feb. 1998.

[116] J. H. Winters and J. Salz, "Upper bounds on the bit-error rate of optimum combining in wireless systems," IEEE Trans. Commun., vol. 46, no. 12, pp. 1619-1624, Dec. 1998.

[117] G. G. Raleigh and J. M. Cioffi, "Spatio-temporal coding for wireless communications," in Proc. 1996 IEEE Global Telecommunications Conf. (GLOBECOM '96), Nov. 18-22, 1996, vol. 3, pp. 1809-1814.

[118] G. G. Raleigh and J. M. Cioffi, "Spatio-temporal coding for wireless communication," IEEE Trans. Commun., vol. 46, no. 3, pp. 357-366, Mar. 1998.

[119] G. J. Foschini and M. J. Gans, "On limits of wireless communications in a fading environment when using multiple antennas," Wireless. Personal Commun., vol. 6, pp. 311-335, Mar. 1998.

[120] G. J. Foschini, G. D. Golden, R. A. Valenzuela, and P. W. Wolniansky, "Simplified processing for high spectral efficiency wireless communication 
employing multi-element arrays," IEEE J. Sel. Areas Commun., vol. 17, no. 11, pp. 1841-1852, Nov. 1999.

[121] B. Lu and X. Wang, "Iterative receivers for multiuser space-time coding systems," IEEE J. Sel. Areas Commun., vol. 18, no. 11, pp. 2322-2335, Nov. 2000.

[122] S. Y. Kung, Y. Wu, and X. Zhang, "Bezout space-time precoders and equalizers for MIMO channels," IEEE Trans. Signal Process., vol. 50, no. 10, pp. 2499-2514, Oct. 2002.

[123] F. Petré, G. Leus, L. Deneire, M. Engels, M. Moonen, and H. D. Man, "Space-time block coding for single-carrier block transmission DS-CDMA downlink," IEEE J. Sel. Areas Commun., vol. 21, no. 3, pp. 350-361, Apr. 2003.

[124] L. Zhang, L. Gui, Y. Qiao, and W. Zhang, "Obtaining diversity gain for DTV by using MIMO structure in SFN," IEEE Trans. Broadcast., vol. 50, no. 1, pp. 83-90, Mar. 2004.

[125] X. Zhu and R. D. Murch, "Layered space-frequency equalization in a single-carrier MIMO system for frequency-selective channels," IEEE Trans. Wireless Commun., vol. 3, no. 3, pp. 701-708, May 2004.

[126] M. R. McKay and I. B. Collings, "Capacity and performance of MIMO-BICM with zero-forcing receivers," IEEE Trans. Commun., vol. 53, no. 1, pp. 74-83, Jan. 2005.

[127] J. Hoadley. (2005, Sep. 19). Building Future Networks With MIMO and OFDM, Telephonyonline.com. [Online]. Available: http://telephonyonline.com/wireless/ technology/mimo_ofdm_091905/

[128] A. J. Paulraj, D. A. Gore, R. U. Nabar, and H. Bölcskei, "An overview of MIMO communications-A key to gigabit wireless," Proc. IEEE, vol. 92, no. 2, pp. 198-218, Feb. 2004.

[129] Datacomm Research Company, Using MIMO-OFDM Technology to Boost Wireless LAN Performance Today, White Paper, St. Louis, MO, Jun. 2005.

[130] H. Sampath, S. Talwar, J. Tellado, V. Erceg, and A. J. Paulraj, "A fourth-generation MIMO-OFDM broadband wireless system: Design, performance, and field trial results," IEEE Commun. Mag., vol. 40, no. 9 , pp. 143-149, Sep. 2002.

[131] Airgo Networks. [Online]. Available: http:// www.airgonetworks.com

[132] H. Bölcskei, D. Gesbert, and A. J. Paulraj, "On the capacity of OFDM-based spatial multiplexing systems," IEEE Trans. Commun. vol. 50, no. 2, pp. 225-234, Feb. 2002.

[133] A. Ganesan and A. M. Sayeed, "A virtual input-output framework for transceiver analysis and design for multipath fading channels," IEEE Trans. Commun., vol. 51, no. 7, pp. 1149-1161, Jul. 2003

[134] R. S. Blum, Y. Li, J. H. Winters, and Q. Yan, "Improved space-time coding for MIMO-OFDM wireless communications," IEEE Trans. Commun., vol. 49, no. 11, pp. 1873-1878, Nov. 2001.

[135] H. E. Gamal, A. R. Hammons, Jr., Y. Liu M. P. Fitz, and O. Y. Takeshita, "On the design of space-time and space-frequency codes for MIMO frequency-selective fading channels," IEEE Trans. Inf. Theory, vol. 49, no. 9, pp. 2277-2292, Sep. 2003.

[136] P. Dayal, M. Brehler, and M. K. Varanasi, "Leveraging coherent space-time codes for noncoherent communication via training,"
IEEE Trans. Inf. Theory, vol. 50, no. 9 pp. 2058-2080, Sep. 2004.

[137] W. Su, Z. Safar, M. Olfat, and K. J. R. Liu, "Obtaining full-diversity space-frequency codes from space-time codes via mapping," IEEE Trans. Signal Process., vol. 51, no. 11, pp. 2905-2916, Nov. 2003.

[138] W. Su, Z. Safar, and K. J. R. Liu, "Full-rat full-diversity space-frequency codes with optimum coding advantage," IEEE Trans. Inf. Theory, vol. 51, no. 1, pp. 229-249, Jan. 2005.

[139] J. H. Moon, Y. H. You, W. G. Jeon, K. W. Kwon, and H. K. Song, "Peak-to-average power control for multiple-antenna HIPERLAN/2 and IEEE802.11a systems," IEEE Trans. Consumer Electron., vol. 49, no. 4, pp. 1078-1083, Nov. 2003.

[140] Y. L. Lee, Y. H. You, W. G. Jeon, J. H. Paik, and H. K. Song, "Peak-to-average power ratio in MIMO-OFDM systems using selective mapping," IEEE Commun. Lett., vol. 7, no. 12, pp. $575-577$, Dec. 2003.

[141] S. H. Han and J. H. Lee, "An overview of peak-to-average power ratio reduction techniques for multicarrier transmission," Wireless Commun., vol. 12, pp. 56-65, Apr. 2005.

[142] Y. Li, "Simplified channel estimation for OFDM systems with multiple transmit antennas," IEEE Trans. Wireless Commun. vol. 1, no. 1, pp. 67-75, Jan. 2002

[143] I. Barhumi, G. Leus, and M. Moonen, "Optimal training design for MIMO OFDM systems in mobile wireless channels," IEEE Trans. Signal Process. vol. 51, no. 6, pp. 1615-1624, Jun. 2003.

[144] M. Shin, H. Lee, and C. Lee, "Enhanced channel-estimation technique for MIMO-OFDM systems," IEEE Trans. Veh. Technol., vol. 53, no. 1, pp. 262-265, Jan. 2004

[145] Y. Li, J. H. Winters, and N. R. Sollenberger, "MIMO-OFDM for wireless communications: Signal detection with enhanced channel estimation," IEEE Trans. Commun., vol. 50, no. 9, pp. 1471-1477, Sep. 2002.

[146] L. Giangaspero, L. Agarossi, G. Paltenghi, S. Okamura, M. Okada, and S. Komaki, "Co-channel interference cancellation based on MIMO OFDM systems," Wireless Commun., vol. 9, pp. 8-17, Dec. 2002.

[147] J. Li, K. B. Letaief, and Z. Cao, "Co-channel interference cancellation for space-time coded OFDM systems," IEEE Trans. Wireless Commun., vol. 2, no. 1, pp. 41-49, Jan. 2003.

[148] S. Y. Park and C. G. Kang, "Complexity-reduced iterative MAP receiver for interference suppression in OFDM-based spatial multiplexing systems," IEEE Trans. Veh. Technol., vol. 53, no. 5, pp. 1316-1326, Sep. 2004.

[149] G. L. Stüber, J. R. Barry, S. W. McLaughlin, Y. Li, M. A. Ingram, and T. G. Pratt, "Broadband MIMO-OFDM wireless communications," Proc. IEEE, vol. 92, no. 2, pp. 271-294, Feb. 2004.

[150] C. Dubuc, D. Starks, T. Creasy, and Y. Hou, "A MIMO-OFDM prototype for next-generation wireless WANs," IEEE Commun. Mag., vol. 42, no. 12 , pp. $82-87$, Dec. 2004.

[151] R. J. Piechocki, P. N. Fletcher, A. Nix, N. Canagarajah, and J. P. McGeehan, "Performance evaluation of BLAST-OFDM enhanced Hiperlan/2 using simulated and measured channel data," Electron. Lett. vol. 37, pp. 1137-1139, Aug. 2001.

[152] C.-N. Chuah, D. N. C. Tse, J. M. Kahn, and R. A. Valenzuela, "Capacity scaling in MIMO wireless systems under correlated fading," IEEE Trans. Inf. Theory, vol. 48, no. 3, pp. 637-650, Mar. 2002.

[153] S. Catreux, V. Erceg, D. Gesbert, and R. W. Heath, Jr., "Adaptive modulation and MIMO coding for broadband wireless data networks," IEEE Commun Mag., vol. 40, no. 6, pp. 108-115, Jun. 2002.

[154] R. Piechocki, P. Fletcher, A. Nix, N. Canagarajah, and J. McGeehan, "A measurement based feasibility study of space-frequency MIMO detection and decoding techniques for next generation wireless LANs," IEEE Trans. Consumer Electron., vol. 48, no. 3, pp. 732-737, Aug. 2002.

[155] A. F. Molisch, M. Z. Win, and J. H. Winters "Space-time-frequency (STF) coding for MIMO-OFDM systems," IEEE Commun. Lett., vol. 6, no. 9, pp. 370-372, Sep. 2002.

[156] A. Stamoulis, S. N. Diggavi, and N. Al-Dhahir, "Intercarrier interference in MIMO OFDM," IEEE Trans. Signal Process., vol. 50, no. 10, pp. 2451-2464, Oct. 2002

[157] A. Doufexi, M. Hunukumbure, A. Nix, M. A. Beach, and S. Armour, "COFDM performance evaluation in outdoor MIMO channels using space/polarisation-time processing techniques," Electron. Lett., vol. 38, pp. 1720-1721, Dec. 2002.

[158] H. Bölcskei, M. Borgmann, and A. J. Paulraj, "Impact of the propagation environment on the performance of space-frequency coded MIMO-OFDM," IEEE J. Sel. Areas Commun. vol. 21, no. 3, pp. 427-439, Apr. 2003.

[159] J. Cai, W. Song, and Z. Li, "Doppler spread estimation for mobile OFDM systems in Rayleigh fading channels," IEEE Trans. Consumer Electron., vol. 49, no. 4, pp. 973-977, Nov. 2003.

[160] G. Leus and M. Moonen, "Per-tone equalization for MIMO OFDM systems," IEEE Trans. Signal Process., vol. 51, no. 11, pp. 2965-2975, Nov. 2003.

[161] R. J. Piechocki, A. Nix, J. P. McGeehan, and S. M. D. Armour, "Joint blind and semi-blind detection and channel estimation," Inst. Electr. Eng.-Communications, vol. 150, pp. 419-426, Dec. 2003.

[162] P. Xia, S. Zhou, and G. B. Giannakis, "Adaptive MIMO-OFDM based on partial channel state information," IEEE Trans. Signal Process., vol. 52, no. 1, pp. 202-213, Jan. 2004.

[163] D. Huang and K. B. Letaief, "Symbol-based space diversity for coded OFDM systems," IEEE Trans. Wireless Commun., vol. 3, pp. 117-127, Jan. 2004.

[164] M. R. G. Butler and I. B. Collings, "A zero-forcing approximate log-likelihood receiver for MIMO bit-interleaved coded modulation," IEEE Commun. Lett., vol. 8, no. 2, pp. 105-107, Feb. 2004.

[165] B. Lu, G. Yue, and X. Wang, "Performance analysis and design optimization of LDPC-coded MIMO OFDM systems," IEEE Trans. Signal Process., vol. 52, no. 2 pp. 348-361, Feb. 2004.

[166] A. V. Zelst and T. C. W. Schenk, "Implementation of a MIMO OFDM-based wireless LAN system," IEEE Trans. Signal Process., vol. 52, no. 2, pp. 483-494, Feb. 2004. 
[167] A. Pascual-Iserte, A. I. Pérez-Neira, and M. A. Lagunas, "On power allocation strategies for maximum signal to noise and interference ratio in an OFDM-MIMO system," IEEE Trans. Wireless Commun., vol. 3, no. 3, pp. 808-820, May 2004.

[168] Y. Zeng and T. S. Ng, "A semi-blind channel estimation method for multiuser multiantenna OFDM systems," IEEE Trans. Signal Process., vol. 52, no. 5, pp. 1419-1429, May 2004.

[169] B. Alien, R. Brito, M. Dohler, and A. H. Aghvami, "Performance comparison of spatial diversity array topologies in an OFDM based wireless LAN," IEEE Trans. Consumer Electron., vol. 50, no. 2, pp. 420-428, May 2004.

[170] J. Tan and G. L. Stüber, "Multicarrier delay diversity modulation for MIMO systems," IEEE Trans. Wireless Commun., vol. 3, no. 5, pp. 1756-1763, Sep. 2004.

[171] X. Wang, Y. R. Shayan, and M. Zeng, "On the code and interleaver design of broadband OFDM systems," IEEE Commun. Lett., vol. 8, no. 11, pp. 653-655, Nov. 2004.

[172] Y. Pan, K. B. Letaief, and Z. Cao, "Dynamic spatial subchannel allocation with adaptive beamforming for MIMO/OFDM systems," IEEE Trans. Wireless Commun., vol. 3, no. 6, pp. 2097-2107, Nov. 2004.

[173] C. Tepedelenlioĝlu and R. Challagulla, "Low-complexity multipath diversity through fractional sampling in OFDM," IEEE Trans. Signal Process., vol. 52, no. 11, pp. 3104-3116, Nov. 2004.

[174] M. S. Baek, M. J. Kim, Y. H. You, and H. K. Song, "Semi-blind channel estimation and PAR reduction for MIMO-OFDM system with multiple antennas," IEEE Trans. Broadcast., vol. 50, no. 4, pp. 414-424, Dec. 2004.

[175] G. Barriac and U. Madhow, "Space-time communication for OFDM with implicit channel feedback," IEEE Trans. Inf. Theory, vol. 50, no. 12, pp. 3111-3129, Dec. 2004.

[176] J. Zhang, A. Kavcic, and K. M. Wong, "Equal-diagonal QR decomposition and its application to precoder design for successive-cancellation detection," IEEE Trans. Inf. Theory, vol. 51, no. 1, pp. 154-172, Jan. 2005.

[177] Y. Yao and G. B. Giannakis, "Blind carrier frequency offset estimation in SISO, MIMO, and multiuser OFDM systems," IEEE Trans. Commun., vol. 53, no. 1, pp. 173-183, Jan. 2005.

[178] K. Zheng, L. Huang, W. Wang, and G. Yang, "TD-CDM-OFDM: Evolution of TD-SCDMA toward 4G," IEEE Commun. Mag., vol. 43, no. 1, pp. 45-52, Jan. 2005

[179] H. Yang, "A road to future broadband wireless access: MIMO-OFDM-based air interface," IEEE Commun. Mag., vol. 43, no. 1, pp. 53-60, Jan. 2005.

[180] Y. Zhang and K. B. Letaief, "An efficient resource-allocation scheme for spatial multiuser access in MIMO/OFDM systems," IEEE Trans. Commun., vol. 53, no. 1, pp. 107-116, Jan. 2005.

[181] X. Ma, M. K. Oh, G. B. Giannakis, and D. J. Park, "Hopping pilots for estimation of frequency-offset and multiantenna channels in MIMO-OFDM," IEEE Trans. Commun., vol. 53, no. 1, pp. 162-172, Jan. 2005.

[182] M. Fozunbal, S. W. McLaughlin, and R. W. Schafer, "On space-time-frequency coding over MIMO-OFDM systems,"
IEEE Trans. Wireless Commun., vol. 4 , no. 1, pp. 320-331, Jan. 2005.

[183] S. Nanda, R. Walton, J. Ketchum, M. Wallace, and S. Howard, "A high-performance MIMO OFDM wireless LAN," IEEE Commun. Mag., vol. 43, no. 2, pp. 101-109, Feb. 2005.

[184] K. J. Kim, J. Yue, R. A. Iltis, and J. D. Gibson, "A QRD-M/Kalman filter-based detection and channel estimation algorithm for MIMO-OFDM systems," IEEE Trans. Wireless Commun., vol. 4, no. 2, pp. 710-721, Mar. 2005.

[185] Y. Qiao, S. Yu, P. Su, and L. Zhang, "Research on an iterative algorithm of LS channel estimation in MIMO OFDM systems," IEEE Trans. Broadcast., vol. 51, no. 1, pp. 149-153, Mar. 2005.

[186] H. Sampath, V. Erceg, and A. Paulraj, "Performance analysis of linear precoding based on field trials results of MIMO-OFDM system," IEEE Trans. Wireless Commun., vol. 4, no. 2, pp. 404-409, Mar. 2005.

[187] F. Rey, M. Lamarca, and G. Vazquez, "Robust power allocation algorithms for MIMO OFDM systems with imperfect CSI," IEEE Trans. Signal Process., vol. 53, no. 3, pp. 1070-1085, Mar. 2005

[188] Y. Sun, Z. Xiong, and X. Wang, "EM-based iterative receiver design with carrier-frequency offset estimation for MIMO OFDM systems," IEEE Trans. Commun., vol. 53, no. 4, pp. 581-586, Apr. 2005.

[189] A. Lodhi, F. Said, M. Dohler, and A. H. Aghvami, "Performance comparison of space-time block coded and cyclic delay diversity MC-CDMA systems," Wireless Commun., vol. 12, pp. 38-45, Apr. 2005.

[190] Z. Wang, Z. Han, and K. J. R. Liu, "A MIMO-OFDM channel estimation approach using time of arrivals," IEEE Trans. Wireless Commun., vol. 4, no. 3, pp. 1207-1213, May 2005.

[191] C. K. Wen, Y. Y. Wang, and J. T. Chen, "A low-complexity space-time OFDM multiuser system," IEEE Trans. Wireless Commun., vol. 4, no. 3, pp. 998-1007, May 2005

[192] W. Su, Z. Safar, and K. J. R. Liu, "Towards maximum achievable diversity in space, time, and frequency: Performance analysis and code design," IEEE Trans. Wireless Commun., vol. 4, no. 4, pp. 1847-1857, Jul. 2005.

[193] M. Tan, Z. Latinović, and Y. Bar-Ness, "STBC MIMO-OFDM peak-to-average power ratio reduction by cross-antenna rotation and inversion," IEEE Commun Lett., vol. 9, no. 7, pp. 592-594, Jul. 2005.

[194] K. W. Park and Y. S. Cho, "An MIMO-OFDM technique for high-speed mobile channels," IEEE Commun. Lett., vol. 9, no. 7, pp. 604-606, Jul. 2005.

[195] L. Shao and S. Roy, "Rate-one space-frequency block codes with maximum diversity for MIMO-OFDM,' IEEE Trans. Wireless Commun., vol. 4, no. 4 pp. 1674-1687, Jul. 2005.

[196] T. C. W. Schenk, X. Tao, P. F. M. Smulders, and E. R. Fledderus, "On the influence of phase noise induced ICI in MIMO OFDM systems," IEEE Commun. Lett., vol. 9, no. 8, pp. 682-684, Aug. 2005.

[197] M. Borgmann and H. Bölcskei, "Noncoherent space-frequency coded MIMO-OFDM," IEEE J. Sel. Areas Commun., vol. 23, no. 9, pp. 1799-1810, Sep. 2005.
[198] A. Tarighat and A. H. Sayed, "MIMO OFDM receivers for systems with IQ imbalances," IEEE Trans. Signal Process., vol. 53, no. 9, pp. 3583-3596, Sep. 2005.

[199] Y. Jiang, J. Li, and W. W. Hager, "Joint transceiver design for MIMO communications using geometric mean decomposition," IEEE Trans. Signal Process., vol. 53, no. 10, pp. 3791-3803, Oct. 2005.

[200] J. Choi and R. W. Heath, Jr., "Interpolation based transmit beamforming for MIMO-OFDM with limited feedback," IEEE Trans. Signal Process., vol. 53, no. 11, pp. 4125-4135, Nov. 2005.

[201] M. S. Baek, H. J. Kook, M. J. Kim, Y. H. You, and H. K. Song, "Multi-antenna scheme for high capacity transmission in the digital audio broadcasting," IEEE Trans. Broadcast., vol. 51, no. 4, pp. 551-559, Dec. 2005.

[202] P. Vandenameele, L. V. D. Perre, and M. Engels, Space Division Multiple Access for Wireless Local Area Networks. London, U.K.: Kluwer, 2001.

[203] I. P. Kovalyov, SDMA for Multipath Wireless Channels: Limiting Characteristics and Stochastic Models, 1st ed. Berlin, Germany: Springer-Verlag, 20043-540-40225-X.

[204] D. Tse and P. Viswanath, Fundamentals of Wireless Communication. Cambridge, U.K.: Cambridge Univ. Press, 200513 978-0-521-84527-4.

[205] M. Cooper and M. Goldburg, "Intelligent antennas: Spatial division multiple access," ArrayComm: Annu. Rev. Commun., pp. 999-1002, 1996.

[206] P. Vandenameele, L. V. D. Perre, M. Engels, B. Gyselinckx, and H. D. Man, "A novel class of uplink OFDM/SDMA algorithms: A statistical performance analysis," in Proc. 1999 IEEE 50th Vehicular Technology Conf. (VTC '99 Fall), Amsterdam, Netherlands, Sep. 19-22, 1999, vol. 1, pp. 324-328.

[207] P. Vandenameele, L. V. D. Perre, M. Engels, B. Gyselinckx, and H. D. Man, "A combined OFDM/SDMA approach," IEEE J. Sel. Areas Commun., vol. 18, no. 11, pp. 2312-2321, Nov. 2000.

[208] S. Thoen, L. V. D. Perre, M. Engels, and H. D. Man, "Adaptive loading for OFDM/SDMA-based wireless networks," IEEE Trans. Commun., vol. 50, no. 11, pp. 1798-1810, Nov. 2002.

[209] S. Thoen, L. Deneire, L. V. D. Perre, M. Engels, and H. D. Man, "Constrained least squares detector for OFDM/ SDMA-based wireless networks," IEEE Trans. Wireless Commun., vol. 2, no. 1 , pp. 129-140, Jan. 2003.

[210] A. T. Alastalo and M. Kahola, "Smart-antenna operation for indoor wireless local-area networks using OFDM," IEEE Trans. Wireless Commun., vol. 2, no. 2, pp. 392-399, Mar. 2003.

[211] M. Y. Alias, A. K. Samingan, S. Chen, and L. Hanzo, "Multiple antenna aided OFDM employing minimum bit error rate multiuser detection," Electron. Lett., vol. 39, pp. 1769-1770, Nov. 2003.

[212] M. Y. Alias, S. Chen, and L. Hanzo, "Multiple-antenna-aided OFDM employing genetic-algorithm-assisted minimum bit-error rate multiuser detection," IEEE Trans. Veh. Technol., vol. 54, no. 5, pp. 1713-1721, Sep. 2005. 
[213] X. Dai, "Carrier frequency offset estimation for OFDM/SDMA systems using consecutive pilots," Inst. Electr. Eng.-Communications, vol. 152, pp. 624-632, Oct. 2005.

[214] Y. S. Yeh and D. Reudink, "Efficient spectrum utilization for mobile radio systems using space diversity," IEEE Trans. Commun., vol. 30, no. 3, pp. 447-455, Mar. 1982

[215] K. T. Ko and B. Davis, "A space-division multiple-access protocol for spot-beam antenna and satellite-switched communication network," IEEE J. Sel. Areas Commun., vol. 1, pp. 126-132, Jan. 1983.

[216] S. C. Swales, M. A. Beach, and D. J. Edwards, "Multi-beam adaptive base-station antennas for cellular land mobile radio systems," in Proc. 1989 IEEE 39th Vehicular Technology Conf. (VTC'89 Spring), May 1-3, 1989, vol. 1 pp. 341-348.

[217] S. C. Swales, M. A. Beach, D. J. Edwards, and J. P. McGeehan, "The performance enhancement of multibeam adaptive base-station antennas for cellular land mobile radio systems," IEEE Trans. Veh. Technol., vol. 39, no. 1, pp. 56-67, Feb. 1990.

[218] B. G. Agee, S. V. Schell, and W. A. Gardner, "Spectral self-coherence restoral: A new approach to blind adaptive signal extraction using antenna arrays," Proc. IEEE, vol. 78, no. 4, pp. 753-767, Apr. 1990.

[219] S. Anderson, M. Millnert, M. Viberg, and B. Wahlberg, "An adaptive array for mobile communication systems," IEEE Trans. Veh. Technol., vol. 40, no. 1, pp. 230-236, Feb. 1991.

[220] P. Balaban and J. Salz, "Optimum diversity combining and equalization in digital data transmission with applications to cellular mobile radio. Part I: Theoretical considerations," IEEE Trans. Commun., vol. 40, no. 5, pp. 885-894, May 1992

[221] P. Balaban and J. Salz, "Optimum diversity combining and equalization in digital data transmission with applications to cellular mobile radio. Part II: Numerical results," IEEE Trans. Commun., vol. 40, no. 5, pp. 895-907, May 1992.

[222] G. Xu, H. Liu, W. J. Vogel, H. P. Lin, S. S. Jeng, and G. W. Torrence, "Experimental studies of space-division-multiple-access schemes for spectral efficient wireless communications," in Proc. 1994 IEEE Int. Conf. Communications (ICC '94), New Orleans, LA, May 1-5, 1994, vol. 2, pp. 800-804.

[223] S. Talwar, M. Viberg, and A. Paulraj, "Blind estimation of multiple co-channel digital signals using an antenna array," IEEE Signal Process. Lett., vol. 1, no. 2, pp. 29-31, Feb. 1994.

[224] A. J. V. D. Veen, S. Talwar, and A. Paulraj, "Blind estimation of multiple digital signals transmitted over FIR channels," IEEE Signal Process. Lett., vol. 2, no. 5, pp. 99-102, May 1995.

[225] B. H. Khalaj, A. Paulraj, and T. Kailath, "Spatio-temporal channel estimation techniques for multiple access spread spectrum systems with antenna arrays," in Proc. 1995 IEEE Int. Conf. Communications (ICC '95), Seattle, WA, Jun. 18-22, 1995, vol. 3, pp. 1520-1524.

[226] K. Anand, G. Mathew, and V. U. Reddy, "Blind separation of multiple co-channel BPSK signals arriving at an antenna array,'
IEEE Signal Process. Lett., vol. 2, no. 9, pp. 176-178, Sep. 1995.

[227] H. Liu and G. Xu, "Smart antennas in wireless systems: Uplink multiuser blind channel and sequence detection," IEEE Trans. Commun., vol. 45, no. 2, pp. 187-199, Feb. 1997.

[228] G. Tsoulos, M. A. Beach, and J. McGeehan, "Wireless personal communications for the 21st century: European technological advances in adaptive antennas," IEEE Commun. Mag., vol. 35, no. 9, pp. 102-109, Sep. 1997.

[229] L. Deneire and D. T. M. Slock, "Blind channel identification based on cyclic statistics," Inst. Electr. Eng.-Radar, Sonar and Navigation, vol. 145, pp. 58-62, Feb. 1998.

[230] G. Tsoulos, J. McGeehan, and M. A. Beach, "Space division multiple access (SDMA) field trials-Part I: Tracking and BER performance," Inst. Electr. Eng.--Radar, Sonar and Navigation, vol. 145, pp. 73-78, Feb. 1998.

[231] G. Tsoulos, J. McGeehan, and M. A. Beach, "Space division multiple access (SDMA) field trials-Part II: Calibration and linearity issues," Inst. Electr. Eng.-Radar, Sonar and Navigation, vol. 145, pp. 79-84, Feb. 1998.

[232] V. A. N. Barroso, J. M. F. Moura, and J. Xavier, "Blind array channel division multiple access (AChDMA) for mobile communications," IEEE Trans. Signal Process., vol. 46, no. 3, pp. 737-752, Mar. 1998.

[233] F. Demmerle and W. Wiesbeck, "A biconical multibeam antenna for space-division multiple access," IEEE Trans. Antennas Propag., vol. 46, no. 6, pp. 782-787, Jun. 1998.

[234] B. Lindmark, S. Lundgren, J. R. Sanford, and C. Beckman, "Dual-polarized array for signal-processing applications in wireless communications," IEEE Trans. Antennas Propag., vol. 46, no. 6, pp. 758-763, Jun. 1998.

[235] B. Suard, G. Xu, H. Liu, and T. Kailath, "Uplink channel capacity of space-division-multiple-access schemes," IEEE Trans. Inf. Theory, vol. 44, no. 4 pp. 1468-1476, Jul. 1998.

[236] S. S. Jeng, G. Xu, H. P. Lin, and W. J. Vogel, "Experimental studies of spatial signature variation at $900 \mathrm{MHz}$ for smart antenna systems," IEEE Trans. Antennas Propag., vol. 46, no. 7, pp. 953-962, Jul. 1998.

[237] P. Petrus, R. B. Ertel, and J. H. Reed, "Capacity enhancement using adaptive arrays in an AMPS system," IEEE Trans. Veh. Technol., vol. 47, no. 3, pp. 717-727, Aug. 1998.

[238] J. M. F. Xavier, V. A. N. Barroso, and J. M. F. Moura, "Closed-form blind channel identification and source separation in SDMA systems through correlative coding," IEEE J. Sel. Areas Commun., vol. 16, no. 8, pp. 1506-1517, Oct. 1998

[239] C. Farsakh and J. A. Nossek, "Spatial covariance based downlink beamforming in an SDMA mobile radio system,' IEEE Trans. Commun., vol. 46, no. 11, pp. 1497-1506, Nov. 1998.

[240] G. V. Tsoulos, "Smart antennas for mobile communication systems: Benefits and challenges," Electron. Commun. Eng. J., vol. 11, pp. 84-94, Apr. 1999.

[241] F. Piolini and A. Rolando, "Smart channel-assignment algorithm for SDMA systems," IEEE Trans. Microw.
Theory Tech., vol. 47, no. 6, pp. 693-699, Jun. 1999.

[242] G. M. Galvan-Tejada and J. G. Gardiner, "Theoretical blocking probability for SDMA," Inst. Electr. Eng.-Communications vol. 146, pp. 303-306, Oct. 1999.

[243] G. M. Galvan-Tejada and J. G. Gardiner, "Theoretical model to determine the blocking probability for SDMA systems," IEEE Trans. Veh. Technol., vol. 50, no. 5, pp. 1279-1288, Sep. 2001.

[244] G. V. Tsoulos, "Experimental and theoretical capacity analysis of space-division multiple access (SDMA) with adaptive antennas," Inst. Electr. Eng.-Communications, vol. 146, pp. 307-311, Oct. 1999.

[245] U. Vornefeld, C. Walke, and B. Walke, "SDMA techniques for wireless ATM," IEEE Commun. Mag., vol. 37, no. 11, pp. 52-57, Nov. 1999.

[246] P. Djahani and J. M. Kahn, "Analysis of infrared wireless links employing multibeam transmitters and imaging diversity receivers," IEEE Trans. Commun., vol. 48 , no. 12 , pp. 2077-2088, Dec. 2000.

[247] F. Shad, T. D. Todd, V. Kezys, and J. Litva, "Dynamic slot allocation (DSA) in indoor SDMA/TDMA using a smart antenna basestation," IEEE/ACM Trans. Netw., vol. 9, no. 1, pp. 69-81, Feb. 2001.

[248] R. Kuehner, T. D. Todd, F. Shad, and V. Kezys, "Forward-link capacity in smart antenna base stations with dynamic slot allocation," IEEE Trans. Veh. Technol. vol. 50, no. 4, pp. 1024-1038, Jul. 2001.

[249] S. S. Jeon, Y. Wang, Y. Qian, and T. Itoh, "A novel smart antenna system implementation for broad-band wireless communications," IEEE Trans. Antennas Propag., vol. 50, no. 5, pp. 600-606, May 2002.

[250] S. Bellofiore, C. A. Balanis, J. Foutz, and A. S. Spanias, "Smart-antenna systems for mobile communication networks - Part 1 : Overview and antenna design," IEEE Trans. Antennas Propag. Mag., vol. 44, no. 3, pp. 145-154, Jun. 2002.

[251] S. Bellofiore, C. A. Balanis, J. Foutz, and A. S. Spanias, "Smart-antenna system for mobile communication networks-Part 2 Beamforming and network throughput,' IEEE Trans. Antennas Propag. Mag., vol. 44, no. 4, pp. 106-114, Aug. 2002.

[252] X. Fang, "More realistic analysis for blocking probability in SDMA systems," Inst. Electr. Eng.-Communications, vol. 149, pp. 152-156, Jun. 2002.

[253] A. Arredondo, K. R. Dandekar, and $\mathrm{G}$. Xu, "Vector channel modeling and prediction for the improvement of downlink received power," IEEE Trans. Commun., vol. 50, no. 7, pp. 1121-1129, Jul. 2002.

[254] C. M. Walke and T. J. Oechtering, "Analytical expression for uplink C/I-distribution in interference-limited cellular radio systems," Electron. Lett., vol. 38, pp. 743-744, Jul. 2002.

[255] T. Zwick, C. Fischer, and W. Wiesbeck, "A stochastic multipath channel model including path directions for indoor environments," IEEE J. Sel. Areas Commun., vol. 20, no. 6, pp. 1178-1192, Aug. 2002.

[256] S. A. Zekavat, C. R. Nassar, and S. Shattil, "Oscillating-beam smart antenna arrays and multicarrier systems: Achieving transmit diversity, frequency diversity, 
and directionality," IEEE Trans. Veh. Technol., vol. 51, no. 5, pp. 1030-1039, Sep. 2002.

[257] J. L. Pan and P. M. Djurić, “Multibeam cellular mobile communications with dynamic channel assignment," IEEE Trans. Veh. Technol., vol. 51, no. 5, pp. 1252-1258, Sep. 2002.

[258] C. C. Cavalcante, F. R. P. Cavalcanti, and J. C. M. Mota, "Adaptive blind multiuser separation criterion based on log-likelihood maximisation," Electron. Lett., vol. 38 , pp. 1231-1233, Sep. 2002.

[259] H. Yin and H. Liu, "Performance of space-division multiple-access (SDMA) with scheduling," IEEE Trans. Wireless Commun., vol. 1, no. 4, pp. 611-618, Oct. 2002.

[260] M. Rim, "Multi-user downlink beamforming with multiple transmit and receive antennas," Electron. Lett., vol. 38, pp. 1725-1726, Dec. 2002.

[261] I. Bradaric, A. P. Pertropulu, and K. I. Diamantaras, "Blind MIMO FIR channel identification based on second-order spectra correlations," IEEE Trans. Signal Process., vol. 51, no. 6, pp. 1668-1674, Jun. 2003.

[262] Q. H. Spencer, A. L. Swindlehurst, and M. Haardt, "Zero-forcing methods for downlink spatial multiplexing in multiuser MIMO channels," IEEE Trans. Signal Process., vol. 52, no. 2, pp. 461-471, Feb. 2004.

[263] J. Li, K. B. Letaief, and Z. Cao, "A reduced-complexity maximum-likelihood method for multiuser detection," IEEE Trans. Commun., vol. 52, no. 2, pp. 289-295, Feb. 2004.

[264] L. U. Choi and R. D. Murch, "A pre-BLAST-DFE technique for the downlink of frequency-selective fading MIMO channels," IEEE Trans. Commun., vol. 52, no. 5, pp. 737-743, May 2004.

[265] W. Ajib and D. Haccoun, "An overview of scheduling algorithms in MIMO-based fourth-generation wireless systems," IEEE Netw., vol. 19, no. 5, pp. 43-48, Sep./Oct. 2005

[266] M. Münster and L. Hanzo, "Parallel-interference-cancellation-assisted decision-directed channel estimation for OFDM systems using multiple transmit antennas," IEEE Trans. Wireless Commun., vol. 4, no. 5, pp. 2148-2162, Sep. 2005.

[267] K. M. Nasr, F. Costen, and S. K. Barton, "A wall imperfection channel model for signal level prediction and its impact on smart antenna systems for indoor infrastructure WLAN," IEEE Trans. Antennas Propag., vol. 53, no. 11, pp. 3767-3775, Nov. 2005.

[268] S. Verdu, Multiuser Detection. Cambridge, U.K.: Cambridge Univ. Press, 1998.

[269] U. Fincke and M. Pohst, "Improved methods for calculating vectors of short length in a lattice, including a complexity analysis," Math. Computation, vol. 44, pp. 463-471, Apr. 1985.

[270] E. Viterbo and J. Boutros, "A universal lattice code decoder for fading channels," IEEE Trans. Inf. Theory, vol. 45, no. 5, pp. 1639-1642, Jul. 1999

[271] M. O. Damen, A. Chkeif, and J.-C. Belfiore, "Lattice code decoder for space-time codes," IEEE Commun. Lett., vol. 4, no. 5, pp. 161-163, May 2000.
[272] B. M. Hochwald and S. ten Brink, "Achieving near-capacity on a multiple-antenna channel," IEEE Trans. Commun., vol. 51, no. 3, pp. 389-399, Mar. 2003.

[273] L. Brunel, "Multiuser detection techniques using maximum-likelihood sphere decoding in multicarrier CDMA systems," IEEE Trans. Wireless Commun., vol. 3, no. 3, pp. 949-957, May 2004.

[274] D. Pham, K. R. Pattipati, P. K. Willet, and J. Luo, "An improved complex sphere decoder for V-BLAST systems," IEEE Signal Process. Lett., vol. 11, no. 9, pp. 748-751, Sep. 2004.

[275] T. Cui and C. Tellambura, "Approximate ML detection for MIMO systems using multistage sphere decoding," IEEE Signal Process. Lett., vol. 12, no. 3, pp. 222-225, Mar. 2005.

[276] J. Akhtman and L. Hanzo, "Reduced-complexity maximum-likelihood detection in multiple-antenna-aided multicarrier systems," in Proc. 5th Int. Workshop Multi-Carrier Spread Spectrum Communications, Oberpfaffenhofen, Germany, Sep. 14-16, 2005.

[277] J. Akhtman and L. Hanzo, "An optimized-hierarchy-aided maximum-likelihood detector for MIMO-OFDM," in Proc. 2006 IEEE 63rd Vehicular Technology Conf. (VTC '06 Spring), Melbourne, Australia, May 7-10, 2006.

[278] P. W. Wolniansky, G. J. Foschini, G. D. Golden, and R. A. Valenzuela, "V-BLAST: An architecture for realizing very high data rates over the rich-scattering wireless channel," in Proc. URSI Int. Symp. Signals, Systems, and Electronics, 1998 (ISSSE '98), Pisa, Italy, Sep. 29-Oct. 2, 1998, pp. 295-300.

[279] M. Jiang and L. Hanzo, "Genetically enhanced TTCM assisted MMSE multi-user detection for SDMA-OFDM," in Proc. 2004 IEEE 60th Vehicular Technology Conf. (VTC '04 Fall), Los Angeles, CA, Sep. 26-29, 2004, vol. 3, pp. 1954-1958.

[280] M. Jiang and L. Hanzo, "Improved hybrid MMSE detection for turbo trellis coded modulation assisted multi-user OFDM systems," Electron. Lett., vol. 40, pp. 1002-1003, Aug. 2004.

[281] M. Jiang and L. Hanzo, "Iterative hybrid multi-user detection using genetic algorithm and biased mutation," in Proc. 2005 IEE 6th Int. Conf. $3 G$ and Beyond (3G '05), London, U.K., Nov. 7-9, 2005, pp. 297-301.

[282] M. Jiang, S. X. Ng, and L. Hanzo, "Hybrid iterative multiuser detection for channel coded space division multiple access OFDM systems," IEEE Trans. Veh. Technol., vol. 55, no. 1, pp. 115-127, Jan. 2006.

[283] M. Jiang, J. Akhtman, and L. Hanzo, "Near-optimum nonlinear soft detection for multiple-antenna assisted OFDM," in Proc. 2006 IEEE Wireless Communications and Networking Conf. (WCNC '06), Las Vegas, NV, Apr. 3-6, 2006.

[284] M. Jiang, J. Akhtman, and L. Hanzo, "Soft-information assisted near-optimum nonlinear detection for BLAST-type space division multiplexing OFDM systems," IEEE Wireless Commun. Lett., vol. 6, no. 4, pp. 1230-1234, Dec. 2006.

[285] J. Xavier, V. A. N. Barroso, and J. M. F. Moura, "Closed-form correlative coding ( $\mathrm{CFC} 2)$ blind identification of MIMO channels: Isometry fitting to second order statistics," IEEE Trans. Signal Process., vol. 49, no. 5, pp. 1073-1086, May 2001.

[286] Y. Li, N. Seshadri, and S. Ariyavisitakul, "Channel estimation for OFDM systems with transmitter diversity in mobile wireless channels," IEEE J. Sel. Areas Commun., vol. 17, no. 3, pp. 461-471, Mar. 1999.

[287] H. Minn, D. I. Kim, and V. K. Bhargava, "A reduced complexity channel estimation for OFDM systems with transmit diversity in mobile wireless channels," IEEE Trans. Commun., vol. 50, no. 5, pp. 799-807, May 2002.

[288] F. W. Vook and T. A. Thomas, "MMSE multi-user channel estimation for broadband wireless communications," in Proc. 2001 IEEE Global Telecommunications Conf. (GLOBECOM '01), San Antonio, TX, Nov. 25-29, 2001, vol. 1, pp. 470-474.

[289] K. J. Kim and R. A. Iltis, "Joint detection and channel estimation algorithms for QS-CDMA signals over time-varying channels," IEEE Trans. Commun., vol. 50, no. 5, pp. 845-855, May 2002.

[290] F. Horlin and L. V. D. Perre, "Optimal training sequences for low complexity ML multi-channel estimation in multi-user MIMO OFDM-based communications," in Proc. 2004 IEEE Int. Conf. Communications (ICC '04), Jun. 20-24, 2004, vol. 4, pp. 2427-2431.

[291] W. Nabhane and H. V. Poor, "Blind joint equalization and multiuser detection in dispersive MC-CDMA/MC-DS-CDMA/ MT-CDMA channels," in Proc. 2002 IEEE Military Communications Conf. (MILCOM '02), Oct. 7-10, 2002, vol. 2, pp. 814-819.

[292] T. Cui and C. Tellambura, "Joint channel estimation and data detection for OFDM systems via sphere decoding," in Proc. 2004 IEEE Global Telecommunications Conf. (GLOBECOM '04), Nov. 29-Dec. 3, 2004, vol. 6, pp. 3656-3660.

[293] H. Zhu, B. Farhang-Boroujeny, and C. Schlegel, "Pilot embedding for joint channel estimation and data detection in MIMO communication systems," IEEE Commun. Lett., vol. 7, no. 1, pp. 30-32, Jan. 2003.

[294] J. Wang and K. Araki, "Pilot-symbol aided channel estimation in spatially correlated multiuser MIMO-OFDM channels," in Proc. 2004 IEEE 60th Vehicular Technology Conf. (VTC '04 Fall), Los Angeles, CA, Sep. 26-29, 2004, vol. 1, pp. 33-37.

[295] J. Siew, J. Coon, R. J. Piechocki, A. Dowler, A. Nix, M. A. Beach, S. Armour, and J. McGeehan, "A channel estimation algorithm for MIMO-SCFDE," IEEE Commun. Lett., vol. 8, no. 9, pp. 555-557, Sep. 2004.

[296] U. Fincke and M. Pohst, "Improved method for calculating vector of short length in a lattice, including a complexity analysis," Math. Comput., vol. 44, pp. 463-471, Apr. 1985.

[297] M. Damen, K. Abed-Meraim, and J.-C. Belfiore, "Generalized sphere decoder for asymmetrical space-time communication architecture," Electron. Lett., vol. 36, no. 2, pp. 166-167, 2000.

[298] T. Cui and C. Tellambura, "An efficient generalised sphere decoder for rank-deficient MIMO systems," IEEE Commun. Lett., vol. 9, no. 5, pp. 423-425, 2005. 
[299] B. Hochwald and S. ten Brink, "Achieving near-capacity on a multiple-antenna channel," IEEE Trans. Commun., vol. 51, no. 3, pp. 389-399, 2003.

[300] M. O. Damen, H. E. Gamal, and G. Caier, "On maximum-likelihood detection and the search for closest lattice point," IEEE Trans. Inf. Theory, vol. 49, no. 10, pp. 2389-2402, Oct. 2003.

[301] D. Pham, K. R. Pattipati, P. K. Willet, and J. Luo, "An improved complex sphere decoder for V-BLAST Systems," IEEE Signal Process. Lett., vol. 11, no. 9, pp. 748-751, Sep. 2004.

[302] T. Cui and C. Tellambura, "Joint channel estimation and data detection for OFDM systems via sphere decoding," in Proc. IEEE Global Telecommunications Conf., Nov. 29-Dec. 3, 2004, vol. 6, pp. 3656-3660.

[303] T. Cui and C. Tellambura, "Approximate ML detection for MIMO systems using multistage sphere decoding," IEEE Signal Process. Lett., vol. 12, no. 3 , pp. 222-225, Mar. 2005.

[304] W. Zhao and G. Giannakis, "Sphere decoding algorithms with improved radius search," IEEE Trans. Commun., vol. 53, no. 7, pp. 1104-1109, Jul 2005.

[305] H. Mühlenbein, Foundations of Genetic Algorithms. San Mateo, CA: Morgan Kaufmann, 1991.

[306] J. J. Grefenstette and J. E. Baker, "How genetic algorithms work: A critical look at implicit parallelism," in Proc. 3rd Int. Conf. Genetic Algorithms, J. D. Schaffer, Ed., San Mateo, CA, 1989, pp. 20-27.

[307] J. J. Grefenstette, Genetic Algorithms for Machine Learning. Norwell, MA: Kluwer Academic, 1993.

[308] H. Adeli and S. L. Hung, Machine Learning: Neural Networks, Genetic Algorithms and Fuzzy Systems. New York: Wiley, 1994.

[309] S. K. Pal and P. P. Wong, Genetic Algorithms for Pattern Recognition. Boca Raton, FL: CRC Press, 1996.

[310] M. D. Vose, The Simple Genetic Algorithm: Foundations and Theory (Complex Adaptive Systems). Cambridge, MA: MIT Press, 1999.

[311] H. Dawid, Adaptive Learning by Genetic Algorithms: Analytical Results and Applications to Economic Models (Lecture Notes in Economics and Mathematical Systems). Berlin, Germany: Springer-Verlag/GmbH \& Co. KG, 1996.

[312] M. Gen and R. Cheng, Genetic Algorithms and Engineering Optimisation, Engineering Design and Automation. New York: Wiley, 2000.

[313] M. J. Juntti, T. Schlösser, and J. O. Lilleberg, "Genetic algorithms for multiuser detection in synchronous CDMA," in Proc. 1997 IEEE Int. Symp. Information Theory (ISIT '97), Ulm, Germany, Jun. 29-Jul. 4, 1997, p. 492.

[314] X. F. Wang, W.-S. Lu, and A. Antoniou, "A genetic-algorithm-based multiuser detector for multiple-access communications," in Proc. 1998 IEEE Int. Symp. Circuits and Systems (ISCAS '98), Monterey, CA, May 31-Jun. 5, 1998, vol. 4, pp. 534-537.

[315] C. Ergün and K. Hacioglu, "Multiuser detection using a genetic algorithm in CDMA communications systems,"
IEEE Trans. Commun., vol. 48, no. 8 , pp. 1374-1383, Aug. 2000.

[316] K. Yen and L. Hanzo,

"Antenna-diversity-assisted genetic-algorithm-based multiuser detection schemes for synchronous CDMA systems," IEEE Trans. Commun., vol. 51, no. 3, pp. 366-370, Mar. 2003.

[317] K. Yen and L. Hanzo, "Genetic algorithm assisted joint multiuser symbol detection and fading channel estimation for synchronous CDMA systems," IEEE J. Sel. Areas Commun., vol. 19, no. 6, pp. 985-998, Jun. 2001.

[318] S. Abedi and R. Tafazolli, "Genetically modified multiuser detection for code division multiple access systems," IEEE J. Sel. Areas Commun., vol. 20, no. 2 , pp. 463-473, Feb. 2002

[319] S. Abedi and R. Tafazolli, "A new CDMA multiuser detection technique using an evolutionary algorithm," Inst. Electr. Eng.: Communications, vol. 148, no. 6, pp. 393-399, Dec. 2001.

[320] M. G. Shayesteh, M. B. Menhaj, and B. G. Nobary, "A modified genetic algorithm for multiuser detection in DS/CDMA," IEICE Trans. Commun., vol. E86-B, no. 8, pp. 2377-2388, Aug. 2003.

[321] S. X. Ng, K. Yen, and L. Hanzo, "TTCM assisted genetic-algorithm aided reduced-complexity multiuser detection," Electron. Lett., vol. 38, no. 14, pp. 722-724, Jul. 4, 2002.

[322] Y. Du and K. T. Chan, "Feasibility of applying genetic algorithms in space-time block coding multiuser detection systems, ' in Proc. IASTED Int. Conf. Wireless and Optical Communications, Banff, AB, Canada, Jul. 2-4, 2003, vol. 3, pp. 469-473.

[323] A. Wolfgang, N. N. Ahmad, S. Chen, and L. Hanzo, "Genetic algorithm assisted error probability optimisation for beamforming," Inst. Electr. Eng. Electron. Lett., vol. 40, no. 5, pp. 320-322, Mar. 4, 2004.

[324] M. Jiang, J. Akhtman, F. Guo, and L. Hanzo, "Iterative joint channel estimation and multi-user detection for high-throughput multiple-antenna aided OFDM systems," in Proc. 2006 IEEE 63rd Vehicular Technology Conf. (VTC '06 Spring), Melbourne, Australia, May 7-10, 2006.

[325] C.-T. Chiang and C.-Y. Chang, "An improved genetic algorithm based on eugenic population for multiuser detection in DS-CDMA systems," in Proc. IEEE Region 10 Annu. Int. Conf. (TENCON), Beijing, China, Oct. 28-31, 2002, vol. 2, pp. 984-987.

[326] A. Wolfgang, N. N. Ahmad, S. Chen, and L. Hanzo, "Genetic algorithm assisted minimum bit-error rate beamforming," in Proc. IEEE Vehicular Technology Conf. (VTC-Spring), Milan, Italy, May 17-19, 2004, pp. 142-146.

[327] M. Y. Alias, S. Chen, and L. Hanzo, "Genetic algorithm assisted minimum bit-error rate multiuser detection in multiple antenna aided OFDM," in Proc. IEEE Vehicular Technology Conf. (VTC-Fall), Los Angeles, CA, Sep. 2004, pp. 548-552.

[328] J. G. Proakis, Digital Communications, 4th ed. New York: McGraw-Hill, 2001.

[329] W. Ledermann and E. Lloyd, Handbook of Applicable Mathematics, Volume II: Probability. New York: Wiley, 1980.

[330] K. Yen and L. Hanzo, "Genetic-algorithm-assisted multiuser detection in asynchronous CDMA communications," IEEE Trans. Veh. Technol., vol. 53, no. 5, pp. 1413-1422, Sep. 2004.

[331] X. Wu, T. C. Chuah, B. S. Sharif, and O. R. Hinton, "Adaptive robust detection for CDMA using a genetic algorithm," Inst. Electr. Eng.-Communications, vol. 150, pp. 437-444, Dec. 10, 2003.

[332] S. Bug, C. Wengerter, I. Gaspard, and R. Jakoby, "Channel modeling based on comprehensive measurements for DVB-T mobile applications," in Proc. 2001 IEEE 18th Instrumentation and Measurement Technology Conf. (IMTC '01), May 21-23, 2001, vol. 1, pp. 544-548.

[333] S. Chen and Y. Wu, "Maximum-likelihood joint channel and data estimation using genetic algorithms," IEEE Trans. Signal Process., vol. 46, no. 5, pp. 1469-1473, May 1998.

[334] Y. S. Zhang, Y. Du, W. Zhang, X. Z. Wang, and J. Li, "A data-aided time domain channel estimation method," in Proc. 2004 Joint Conf. 10th Asia-Pacific Conf. Communications/5th Int. Symp. Multi-Dimensional Mobile Communications, Aug. 29-Sep. 1, 2004, vol. 1, pp. 469-473.

[335] C. E. Tan and I. J. Wassell, "Near-optimum training sequences for OFDM systems," in Proc. 9th Asia-Pacific Conf. Communications (APCC '03), Sep. 21-24, 2003, vol. 1 , pp. 119-123.

[336] T. Bäck, Evolutionary Algorithms in Theory and Practice: Evolution Strategies, Evolutionary Programming, Genetic Algorithms. New York: Oxford Univ. Press, 1996.

[337] D. B. Fogel, “An introduction to simulated evolutionary optimization," IEEE Trans. Neural Netw., vol. 5, no. 1, pp. 3-14, Jan. 1994.

[338] S. Haykin, Neural Netw., 2nd ed. Upper Saddle River, NJ: Prentice-Hall, 1999.

[339] L. Fausett, Fundamentals of Neural Networks: Architectures, Algorithms and Applications. Upper Saddle River, NJ: Prentice-Hall, 1994.

[340] M. D. Buhmann, Radial Basis Functions: Theory and Implementations. Cambridge, U.K.: Cambridge Univ. Press, 2003.

[341] P. V. Yee and S. Haykin, Regularized Radial Basis Function Networks: Theory and Applications. New York: Wiley, 2001.

[342] L. Hanzo, C. H. Wong, and M. S. Yee, Adaptive Wireless Tranceivers. Piscataway, NJ: IEEE Press/Wiley, 2002.

[343] U. Mitra and H. V. Poor, "Neural network techniques for adaptive multiuser demodulation," IEEE J. Sel. Areas Commun., vol. 12, no. 9, pp. 1460-1470, Dec. 1994.

[344] K. Ko, S. Choi, C. Kang, and D. Hong, "RBF multiuser detector with channel estimation capability in a synchronous MC-CDMA system," IEEE Trans. Neural Netw., vol. 12, no. 6, pp. 1536-1539, Nov. 2001.

[345] C. Ahn and I. Sasase, "Adaptive array antenna based on radial basis function network as multiuser detection for WCDMA," Electron. Lett., vol. 38, pp. 1208-1210, Sep. 2002.

[346] X. Zhou and X. Wang, "Channel estimation for OFDM systems using adaptive radia basis function networks," IEEE Trans. Veh. Technol., vol. 52, no. 1, pp. 48-59, Jan. 2003. 


\section{ABOUT THE AUTHORS}

Ming Jiang (Member, IEEE) received the B.Eng. and M.Eng. degrees in electronics engineering from South China University of Technology (SCUT), China, in 1999 and 2002, respectively, and the Ph.D. degree in telecommunications from University of Southampton, U.K., in 2006.

From 2002 to 2005, he was involved in the Core 3 research project of the Mobile Virtual Centre of Excellence (VCE), U.K. on air-interface algorithms designed for MIMO OFDM systems.

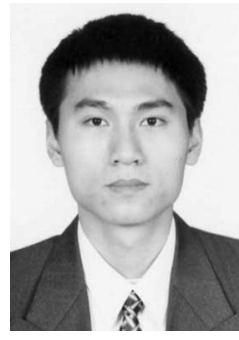

Since April 2006, he has been with Advanced Technology, Standards and Regulation (ATSR) of Samsung Electronics Research Institute (SERI), U.K., working on the European FP6 WINNER project as well as on internal projects related to advanced wireless communication systems. His research interests fall in the general area of wireless communications, including multiuser detection, channel estimation, space-time processing, heuristic and adaptive optimization, frequency-hopping, MIMO OFDM and OFDMA systems, etc. He has co-authored one IEEE Press book chapter, 6 IEE/IEEE journal papers, and 8 IEE/IEEE conference papers.
Lajos Hanzo (Fellow, IEEE) received the firstclass degree in electronics in 1976 and the doctorate degree in 1983, both from the Technical University of Budapest, Hungary. In 2004, he was awarded the D.Sc. degree from the University of Southampton, Southampton, U.K.

During his career in telecommunications he has held various research and academic posts in Hungary, Germany, and the U.K. Since 1986 he has been with the Department of Electronics and

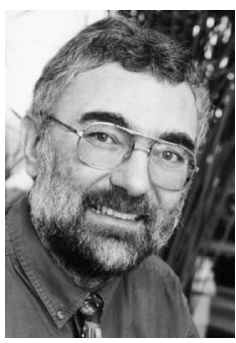
Computer Science, University of Southampton, where he holds the chair in telecommunications. He co-authored 15 books totalling 10000 pages on mobile radio communications, published about 700 research papers, acted as TPC Chair of IEEE conferences, presented various keynote and overview lectures, and has been awarded a number of distinctions. Currently, he heads an academic research team, working on a range of research projects in the field of wireless multimedia communications sponsored by industry, the Engineering and Physical Sciences Research Council (EPSRC) U.K., the European IST Programme and the Mobile Virtual Centre of Excellence (VCE), U.K. He is an enthusiastic supporter of industrial and academic liaison and he offers a range of industrial courses.

Dr. Hanzo is a Fellow of the Royal Academy of Engineering. He is also an IEEE Distinguished Lecturer of both the Communications as well as the Vehicular Technology Society, a Fellow of the IEE/IET. He is an Editorial Board member of the PROCEEDINGS OF THE IEEE and a Governor of the IEEE VT Society. For further information on research in progress and associated publications please refer to http://www-mobile.ecs. soton.ac.uk. 